\title{
Superpotentials of D-branes in Calabi-Yau manifolds with several moduli by mirror symmetry and blown-up
}

\author{
Xuan Li, Yuan-Chun Jing and Fu-Zhong Yang ${ }^{1}$ \\ School of Physical Sciences, University of Chinese Academy of Sciences, \\ No. 19(A) Yuquan Road, Shijingshan District, Beijing 100049, P.R. China \\ E-mail: lixuan191@mails.ucas.ac.cn, jingyuanchun18@mails.ucas.ac.cn, \\ fzyang@ucas.ac.cn
}

ABSTRACT: We study B-brane superpotentials depending on several closed- and openmoduli on Calabi-Yau hypersurfaces and complete intersections. By blowing up the ambient space along a curve wrapped by B-branes in a Calabi-Yau manifold, we obtain a blow-up new manifold and the period integral satisfying the GKZ-system. Via mirror symmetry to A-model, we calculate the superpotentials and extract Ooguri-Vafa invariants for concrete examples of several open-closed moduli in Calabi-Yau manifolds.

Keywords: D-Branes, Flux Compactifications, Topological Strings

ARXIV EPRINT: 2201.00322

\footnotetext{
${ }^{1}$ Corresponding author.
} 


\section{Contents}

1 Introduction 1

2 Toric geometry and blowing up 2

2.1 Toric geometry and GKZ system 2

2.2 Blowing up and Hodge structure 5

3 Two closed and two open moduli case 7

3.1 Open-closed GKZ-system: branes on $X_{9}^{(1,1,1,3,3)} \quad 7$

$\begin{array}{lll}3.1 .1 & \text { Five branes wrapping lines } & 7\end{array}$

$\begin{array}{lll}3.1 .2 & \text { Toric branes and blowing up geometry } & 9\end{array}$

$\begin{array}{lll}3.1 .3 & \text { Brane superpotential and disk instantons } & 12\end{array}$

$\begin{array}{ll}\text { 3.1.4 Another curve on X } & 13\end{array}$

3.2 Open-closed GKZ-system: branes on $X_{8}^{(1,1,2,2,2)} 14$

3.2.1 Branes wrapping rational curves and blowing up geometry 14

$\begin{array}{ll}\text { 3.2.2 Brane superpotential and disk instantons } & 17\end{array}$

3.3 Open-closed GKZ-system: branes on $X_{12}^{(1,1,2,2,6)} 18$

3.3.1 Five branes wrapping rational curves and blowing up geometry 18

$\begin{array}{lll}\text { 3.3.2 } & \text { Brane superpotential and disk instantons } & 21\end{array}$

4 One closed and two open moduli case $\quad 22$

4.1 Open-closed GKZ-system: branes on complete intersections $\mathbb{P}_{[3,3]}^{(111 \mid 111)} \quad 22$

4.1.1 Five branes wrapping lines and blowing up geometry 22

$\begin{array}{lll}\text { 4.1.2 Brane superpotential and disk instantons } & 25\end{array}$

4.2 Branes on complete intersections $\mathbb{P}_{[4,4]}^{(112 \mid 112)}$ and $\mathbb{P}_{[6,6]}^{(123 \mid 123)} \quad 26$

5 One closed and three open moduli case $\quad 26$

5.1 Open-closed GKZ-system: branes on sextic hypersurface 26

5.1.1 Branes wrapping rational curves and blowing up geometry 26

5.1.2 Brane superpotential and disk instantons 28

5.2 Open-closed GKZ-system: branes on sextic hypersurface 29

5.2.1 Branes wrapping rational curves and blowing up geometry 29

$\begin{array}{ll}\text { 5.2.2 Brane superpotential and disk instantons } & 30\end{array}$

$\begin{array}{lll}6 & \text { Summary and conclusions } & 32\end{array}$

A Blow-up geometry of $X_{[4,4]}^{(112 \mid 112)}$ and $X_{[6,6]}^{(123 \mid 123)} \quad 32$

A.1 $X_{[4,4]}^{(112 \mid 112)} \quad 32$

A.2 $X_{[6,6]}^{(123 \mid 123)}-35$ 
B Ooguri-Vafa invariants for two closed and two open moduli

B.1 Ooguri-Vafa invariants for $X_{9}^{(1,1,3,3,3)} \quad 38$

B.2 Ooguri-Vafa invariants for $X_{8}^{(1,1,2,2,2)} 41$

B.3 Ooguri-Vafa invariants for $X_{12}^{(1,1,2,2,6)} 42$

C Ooguri-Vafa invariants for one closed and two open moduli 46

$\begin{array}{ll}\text { D Ooguri-Vafa invariants for one closed and three open moduli } & 47\end{array}$

\section{Introduction}

The type IIB compactification with branes can be described by an effective $N=1$ supergravity theory with a non-trivial superpotential on the open-closed moduli space, because the D-branes wrapping supersymmetric cycles reduce the $\mathrm{N}=2$ supersymmetry to $\mathrm{N}=1$ supersymmetry. In topological string theory, there are two type D-branes. A-branes wrap special lagrangian cycles and B-branes wrap holomorphic cycles that can be even dimensional in a Calabi-Yau threefold.

On the A-model side, the superpotential is related to the topological string amplitudes, which counts disk instantons [1,2]. On the B-model side, the topological string is related to holomorphic Chern-Simons theory [3]. The B-brane superpotential is given by a integral over 3-chain with boundary consisting of 2-cycles $\gamma$ around the B-branes, which is the sections of a line bundle over the open-closed moduli space described by the holomorphic $\mathrm{N}=1$ special geometry [4-6]. The B-brane superpotential can be expressed as a linear combination of the integral of the basis of relative period. When considering B-brane wrapping two curves within the same homology class, the superpotential changes on the two sides of the domain wall whose tension is in terms of the Able-Jacobi map.

In physics, the D-brane superpotential is topological sector of the $\mathrm{d}=4, \mathrm{~N}=1$ superpymmetric spacetime effective Lagrangian, as well as the generating function of open string topological field theory correlators. It encode the instanton correction and its derivatives determine structure constants of $2 \mathrm{~d}$ chiral ring and Gauss-Manin connection of the vacuum bundle on the moduli space. The flatness of this connection determines the Picard-Fuchs equations satisfied by period vector. The expansion of superpotentials at large volume phase underlies the Ooguri-Vafa invariants counting the holomorphic disks ending on a lagrangian submanifold on the A-model side. These invariants closely relate to space of the states, non-pertubative effects, and geometric properties of the moduli space.

From the perspective of the deformation theory, the deformations of a curve in CalabiYau threefold are given by the sections of the normal bundle. The holomorphic sections lead to massless or light fields in the effective theory and the non-holomorphic sections lead to massive fields whose masses are given by the volume change under infinitesimal deformations. The superpotentials are related to the deformations with masses vanishing at some point in the closed moduli space. In order words, determining the B-brane superpotentials 
is equivalent to solve the deformation theory of a pair $(X, S)$ with curve $S$ and Calabi-Yau manifold $X$. When a D5 brane wrapping a rational curve, the non-trivial superpotential is defined on a family of curves $S$, whose members are in general non-holomorphic except at some critical points where $S$ is holomorphic curve. The critical locus corresponds to the supersymmetric vacus, other points in moduli space correspond to the obstructed deformation of the rational curve and excitation about the supersymmetric minimum.

The computation of off-shell superpotential for a toric brane, has been presented by local case in $[4,5,7]$, and extended to compact Calabi-Yaus in [8-10]. For this brane, the onshell superpotentials and flat coordinates are the solutions to a system of open-closed Picard-Fuchs equations, which arise as a consequence of the $\mathrm{N}=1$ special geometry. These equation can be obtained by Griffish-Dwork reduction method or GKZ system. When a B-brane wrapping a curve $S$ in $X$, the blowing up $X$ along $S$ lead to a new manifold $X^{\prime}$ with an exceptional divisor $E[11,12]$. Meanwhile, the deformation theory of $(X, S)$ is equivalent to that of $\left(X^{\prime}, E\right) .{ }^{1}$ The B-brane superpotential on the Calabi-Yau threefold $X$ can be calculated in terms of period vector of manifold $X^{\prime}$. In this note, we first calculate Bbrane superpotentials in Calabi-Yau manifolds with several moduli via blowing up method, then extract Ooguri-Vafa invariants from A-model side at large volume phase by mirror symmetry.

The organization of this paper is as follows. In section 2, we introduce the background and formalism. To begin with, we review D-brane superpotential in the Type II string theory and relative cohomology description, recall the basic toric geometry about constructing Calabi-Yau manifold, generalized GKZ system and its local solutions, and outline the procedure to blow up a curve on a Calabi-Yau manifold. In section 3-5, for degree $-9,-8$, -12 Calabi-Yau hypersurface and degree- $(3,3)$ complete intersection Calabi-Yau manifold, we apply the blow up method to the mainfold with a curve on it and obtain a new Kahler manifold an exceptional divisor. The Picard-Fuchs equations and their solutions are derived by GKZ hypergeometric system from toric data of the enhanced polyhedron. The superpotential are identified as double-logarithmic solutions of the Picard-Fuchs equations and Ooguri-Vafa invariants are extracted at large volume phase. The last section is a brief summary and further discussions. In appendix A, we summarize the GKZ-system for two complete intersections Calabi-Yau manifolds $X_{[4,4]}^{(112 \mid 112)}$ and $X_{[6,6]}^{(123 \mid 123)}$. In appendix B, we present the compact instanton invariants of above models for first several orders.

\section{Toric geometry and blowing up}

\subsection{Toric geometry and GKZ system}

A Calabi-Yau manifold can be defined as a hypersurface or complete intersection of several hyperserfaces in ambient toric variety. We refer to [13] for the background of toric geometry, construction of Calabi-Yau manifolds [14, 15], and GKZ system [16-20].

For a mirror pair of compact hypersurfaces $\left(X^{*}, X\right)$, one may associate a pair of integral polyhedra $\left(\Delta^{*}, \Delta\right)$ in a four-dimensional integral lattice and its dual. The $\mathrm{n}$ integral points

\footnotetext{
${ }^{1}$ Section 4.4 in [12] and footnote 17 in [11].
} 
of the polyhedron correspond to homogeneous coordinates $x_{i}$ on the toric ambient space and satisfy linear relations

$$
\sum_{i} l_{i}^{j} v_{i}=0, \quad a=1, \ldots, h^{2,1}
$$

where $l_{i}^{j}$ is the ith component of the charge vector $l^{j}$. The integral entries of the vectors $l^{j}$ define the weights $l_{i}^{j}$ of the coordinates $x_{i}$ under the $\mathbb{C}^{*}$ action

$$
x_{i} \rightarrow\left(\lambda_{j}\right)^{l_{i}^{j}} x_{i}, \quad \lambda_{j} \in \mathbb{C}^{*}
$$

and $l_{i}^{j}$ 's are the U(1) charges of the fields in the gauged linear sigma model (GLSM) [21]. In above description, the mirror Calabi-Yau threefold is determined as a hypersurface in the dual toric ambient space with constraints

$$
P=\sum_{i} a_{i} y_{i}, \quad \prod_{i} y_{i}^{l_{i}^{j}}=z_{j}
$$

Here $z_{j}$ denotes the complex structure moduli of $X$. In terms of vertices $v_{j}^{*} \in \Delta^{*}, v_{i} \in \Delta$

$$
P(x ; a)=\sum_{v_{j}^{*} \in \Delta^{*}} a_{j} \prod_{i} x_{i}^{\left\langle v_{j}^{*}, v_{i}\right\rangle+1}
$$

The complete intersection Calabi-Yau threefolds can be constructed similarly and we omit the details. Equivalently, Calabi-Yau hypersurfaces is also given by the zero loci of certain sections of the anticanonical bundle. The toric variety contains a canonical Zariski open torus $\mathbb{C}^{4}$ with coordinates $X=\left(X_{1}, X_{2}, X_{3}, X_{4}\right)$. The sections are

$$
P_{\Delta^{*}}(X, a)=\sum_{v_{i}^{*} \in \Delta^{*} \cap \mathbb{Z}^{5}} a_{i} X^{v_{i}^{*}}
$$

After homogenization, above equation is the same as (2.2). On these manifolds, a mirror pairs of branes, defined in [7] by another $\mathrm{N}$ charge vectors $\hat{l}^{j}$. The special Lagrangian submanifold wrapped by the A-brane is described in terms of the vectors $\hat{l}^{j}$ satisfying

$$
\sum_{i} \hat{l}_{i}^{j}\left|x_{i}\right|^{2}=c_{j}, \quad \sum_{i} \hat{l}_{i}^{j}=0
$$

where $c_{j}$ parametrize the brane position. And the holomorphic submanifold wrapped by mirror B-brane is defined by the following equation

$$
\prod_{i} y_{i}-\hat{z}_{a}=0, \quad \hat{z}_{a}=\epsilon_{a} e^{-c_{a}}
$$

The $N=2$ case, a toric curve, is more interesting to us and it is the geometry setting we are studying in this notes. To handle the toric curve case, we consider the enhanced polyhedron method proposed in [8]. It is possible in a simple manner to construct the enhanced polyhedron from the original polyhedra and the toric curve specified by two charge vectors. We denote the vertices of $\Delta$ by $v_{i}, i=1, \ldots, n$, with $v_{0}$ the origin, its 
charge vectors by $l^{i}$, and two brane vectors by $\hat{l}^{1}$ and $\hat{l}^{2}$. We add 4 points to $\Delta^{*}$ to define a new polyhedron $\Delta^{\prime}$ with vertices

$$
\begin{aligned}
& X: \quad v_{i}=\left(1,0, v_{i}, 0\right), \quad i=0, \ldots, n \\
& \hat{l}^{1}: \quad v_{n+1}=\left(0,1, v_{1}^{-},-1\right), \quad v_{n+2}=\left(0,1, v_{1}^{+},-1\right) \\
& \hat{l}^{2}: v_{n+3}=\left(0,1, v_{2}^{-},-1\right), \quad v_{n+4}=\left(0,1, v_{2}^{+},-1\right)
\end{aligned}
$$

where we use the abbreviation

$$
\begin{array}{ll}
v_{1}^{+}=\sum_{\hat{l}_{i}^{1}>0} \hat{l}_{i}^{1} v_{i}, & v_{1}^{-}=-\sum_{\hat{l}_{i}^{1}<0} \hat{l}_{i}^{1} v_{i} \\
v_{2}^{+}=\sum_{\hat{l}_{i}^{2}>0} \hat{l}_{i}^{2} v_{i}, & v_{2}^{-}=-\sum_{\hat{l}_{i}^{2}<0} \hat{l}_{i}^{2} v_{i}
\end{array}
$$

The first line of (2.4) simply embeds the original toric data associated to $\Delta$ into $\Delta^{\prime}$, whereas the second and third line translate the brane data into geometric data of $\Delta^{\prime}$.

Given the toric data, the GKZ-system on the complex structure moduli space of $X$ is given by the standard formula

$$
\begin{aligned}
\mathcal{L}_{i} & =\prod_{l_{j}^{i}>0}\left(\frac{\partial}{\partial a_{j}}\right)^{l_{j}^{i}}-\prod_{l_{j}^{i}<0}\left(\frac{\partial}{\partial a_{j}}\right)^{-l_{j}^{i}}, & & i=1, \ldots, h^{2,1}+2 \\
\mathcal{Z}_{i} & =\sum_{j}\left(\bar{v}_{j}\right)^{i} \vartheta_{j}-\beta_{i}, & j & =0, \ldots, 6
\end{aligned}
$$

Here $\beta=(-1,0,0,0,0)$ is the so-called exponent of GKZ system, $\vartheta_{j}=a_{j} \frac{\partial}{\partial a_{j}}$ are the logarithmic derivative and $\bar{v}_{j}=\left(1, v_{j}\right)$. The operators $\mathcal{L}_{i}$ 's express the trivial algebraic relations among monomials entering hypersurface constraints, $\mathcal{Z}_{0}$ expresses the infinitesimal generators of overall rescaling, $\mathcal{Z}_{i}, i \neq 0$ 's eare the infinitesimal generators of rescalings of coordinates $x_{j}$. All GKZ operators can annihilate the period matrix, thus determine the mirror maps and superpotentials.

This immediately yields a natural choice of complex coordinates given by

$$
z^{j}=(-)^{l_{0}^{j}} \prod_{i} a_{i}^{l_{i}^{j}}
$$

And from the operators $\mathcal{L}_{i}$, it is easy to obtain a complete set of Picard-Fuchs operators $\mathcal{D}_{i}$. Using monodromy information and knowledge of the classical terms, their solution can be associated to integrals over an integral basis of cycles in $H^{3}(X, \mathbb{Z})$ and given the flux quanta explicit superpotentials can be written down.

For appropriate choice of basis vector $l^{j}$, solutions to the GKZ system can be written in terms of the generating functions in these variables

$$
\varpi(z ; \rho)=\sum \frac{\Gamma\left(1-\sum_{j} l_{0}^{j}\left(n_{j}+\rho_{j}\right)\right)}{\prod_{i>0} \Gamma\left(1+\sum_{j} l_{i}^{j}\left(n_{j}+\rho_{j}\right)\right)} \prod_{k} z_{k}^{n_{j}+\rho_{j}}
$$


then we have a natural basis for the period vector

$$
\begin{aligned}
\omega_{0}(z) & =\left.\varpi(z ; \rho)\right|_{\rho \rightarrow 0}, \\
\omega_{1, i}(z) & =\left.\partial_{\rho_{i}} \varpi(z ; \rho)\right|_{\rho \rightarrow 0}, \\
\omega_{2, i}(z) & =\left.\sum_{j, k} K_{i j k} \partial_{\rho_{j}} \partial_{\rho_{k}} \varpi(z ; \rho)\right|_{\rho \rightarrow 0} \\
& \ldots
\end{aligned}
$$

For a maximal triangulation corresponding to a large complex structure point centered at $z=0, \forall a, \omega_{0}(z)=1+\mathcal{O}(z)$ and $\omega_{1, i}(z) \sim \log \left(z_{i}\right)$ that define the open-closed mirror maps

$$
t_{i}(z)=\frac{\omega_{1, i}(z)}{\omega_{0}(z)}=\frac{1}{2 \pi i} \log \left(z_{i}\right)+S(z), \quad q_{i}=e^{2 \pi i t_{i}}
$$

where $S(z)$ is a series in the coordinates $z$. In addition, the special solution $\Pi=\mathcal{W}_{\text {open }}(z)$ has further property that its instanton expansion near a large volume/large complex structure point encodes the Ooguri-Vafa invariants of the brane geometry.

$$
\mathcal{W}_{\text {inst }}(q)=\sum_{\beta} G_{\beta} q^{\beta}=\sum_{\beta} \sum_{k=1}^{\infty} N_{\beta} \frac{q^{k \cdot \beta}}{k^{2}}
$$

\subsection{Blowing up and Hodge structure}

Blowing up in algebraic geometry is an important tool in this work. Now, we review the construction and properties of blowing up a manifold along its submanifold. Given $S$ be a curve in a Calabi-Yau threefold $X \in Z$ with $Z$ an ambient toric variety, we can blow up along $S$ to obtain a new manifold.

According to section $2.2(\mathrm{~d})$ in [22], for this case that $X \subset Z$ is a closed irreducible non-singular subvariety of $Z$ and $X$ is transversal to $S$ at every point $S \cap X, \pi: Z^{\prime} \rightarrow Z$ be the blowup of $S$. Then the subvariety $\pi^{-1}(X)$ consist of two irreducible components,

$$
\pi^{-1}(X)=\pi^{-1}(S \cap X) \cup X^{\prime}
$$

and $\pi: X^{\prime} \rightarrow X$ defines the blow-up of $X$ with center in $S \cap X=S$, i.e. $X^{\prime}$ is the manifold obtained from blowing up $X$ along $S$. The subvariety $X^{\prime} \subset Z^{\prime}$ is called the birational transform of $X \subset Z$ under the blowup.

First, by the local construction, we consider an three dimensional multidisk in $X, \Delta$ with holomorphic coordinates $x_{i}, i=1,2,3$, and $V$ is specified by $x_{1}=x_{2}=0$ on each $\Delta$. Then we define the smooth variety

$$
\tilde{\Delta} \subset \Delta \times \mathbb{P}^{1}
$$

as follows

$$
\tilde{\Delta}=\left\{\left(x_{1}, x_{2}, x_{3},\left(y_{1}: y_{2}\right)\right) \subset \Delta \times \mathbb{P}^{1}: x_{2} y_{1}-x_{1} y_{2}=0\right\}
$$

Here $y_{1}, y_{2}$ are the homogeneous coordinates on $\mathbb{P}^{1}$. The projection map $\pi: \tilde{\Delta} \rightarrow \Delta$ on the first factor is clearly an isomorphism away from $V$, while the inverse image of a point 
$z \in V$ is a projective space $\mathbb{P}^{1}$. The manifold $\tilde{\Delta}$, together with the projection map $\pi$ is the blow-up of $\Delta$ along $V$; the inverse image $E=\pi^{-1}(V)$ is an exceptional divisor of the blow-up. For two coordinates patches $U_{i}=\left(y_{i} \neq 0\right), i=1,2$, they have holomorphic coordinates respectively

$$
\begin{array}{rlrl}
z_{1}^{(1)} & =x_{1}, & z_{2}^{(1)}=\frac{y_{2}}{y_{1}}=\frac{x_{2}}{x_{1}}, & y_{3}=x_{3} \\
z_{1}^{(2)}=\frac{y_{1}}{y_{2}}=\frac{x_{1}}{x_{2}}, & z_{2}^{(1)}=x_{2}, & y_{3}=x_{3}
\end{array}
$$

with transition function on $U_{1} \cap U_{2}$ given by $g_{i j}=z_{i}^{(j)}=\frac{y_{i}}{y_{j}}=\frac{x_{i}}{x_{j}}$. Next we consider the global construction of the blow-up manifold. Let $X$ be a complex manifold of dimension three and $S \subset X$ be a curve. Let $\left\{U_{\alpha}\right\}$ be a collection of disks in $X$ covering $S$ such that in each disk $\Delta_{\alpha}$ the subvariety $S \cap \Delta_{\alpha}$ may be given as the locus $\left(x_{1}=x_{2}=0\right)$, and let $\pi_{\alpha}: \tilde{\Delta}_{\alpha} \rightarrow \Delta_{\alpha}$ be the blow-up of $\Delta_{\alpha}$ along $S \cap \Delta_{\alpha}$. We then have

$$
\pi_{\alpha \beta}: \pi_{\alpha \beta}^{-1}\left(U_{\alpha} \cap U_{\beta}\right) \rightarrow \pi_{\beta}^{-1}\left(U_{\alpha} \cap U_{\beta}\right)
$$

and using them, we can patch together the local blow-ups $\tilde{\Delta}_{\alpha}$ to form a manifold

$$
\tilde{\Delta}=\cup_{\pi_{\alpha \beta}} \tilde{\Delta}_{\alpha}
$$

Finally, sinve $\pi$ is an isomorphism away from $X \cap\left(\cup \Delta_{\alpha}\right)$, we can take

$$
X^{\prime}=\tilde{\Delta} \cup_{\pi} X-S
$$

$X^{\prime}$, together with the projection map $\pi: X^{\prime} \rightarrow X$ extending $\pi$ on $\tilde{\Delta}$ and the identity on $X-S$, is called the blow-up of $X$ along $S$, and the inverse image $\pi^{-1}(S)$ is an exceptional divisor.

From the excision theorem of cohomology in algebraic topology [23],

$$
H^{3}(X, S) \cong H^{3}(X-S) \cong H^{3}\left(X^{\prime}-E\right) \cong H^{3}\left(X^{\prime}, E\right)
$$

which means that the variation of the mixed Hodge structures of $H^{3}(X, S)$ and $H^{3}\left(X^{\prime}, E\right)$ over the corresponding moduli space are equivalent. The mixed Hodge structure as follow,

$$
\phi: H^{3}\left(X^{\prime}-E\right) \stackrel{\sim}{\longrightarrow} \oplus_{p+q=3} H^{q}\left(X^{\prime}, \Omega^{\prime}\right)
$$

where $\Omega^{\prime}$ denotes the holomorphic p-forms on $X^{\prime}$. The filtrations have the form

$$
F^{m} H^{3}=\oplus_{p \leq m} H^{3-p}\left(X^{\prime}, \Omega^{\prime}\right)
$$

and

$$
W_{-1} H^{3}=0, \quad W_{0} H^{3}=H^{3}\left(X^{\prime}\right), \quad W_{1} H^{3}=H^{3}\left(X^{\prime}-E\right)
$$

Additionally, the mixed Hodge structure has graded weights

$$
G r_{k}^{W} H^{3}=G r_{-k+3}^{W} H^{3} / G r_{-(k+1)+3}^{W} H^{3}
$$


that take the following form for the divisor $\mathrm{E}$

$$
\begin{aligned}
& G r_{3}^{W} H^{3}=G r_{0}^{W} H^{3} / G r_{-1}^{W} H^{3} \cong H^{3}\left(X^{\prime}\right), \\
& G r_{2}^{W} H^{3}=G r_{1}^{W} H^{3} / G r_{0}^{W} H^{3} \cong H^{2}(E)
\end{aligned}
$$

The reason to consider these (graded) weights is the following: the mixed Hodge structure is defined such that the Hodge filtration $F^{m} H^{3}$ induces a pure Hodge structure on each graded weight, i.e. on $G r_{2}^{W} H^{3}$ and $G r_{3}^{W} H^{3}$. Thus, the following two induced filtrations on $G r_{3}^{W} H^{3}$

$$
H^{3}\left(X^{\prime}\right) \cap F^{3} H^{3} \subset H^{3}\left(X^{\prime}\right) \cap F^{2} H^{3} \subset H^{3}\left(X^{\prime}\right) \cap F^{1} H^{3} \subset H^{3}\left(X^{\prime}\right) \cap F^{0} H^{3}=H^{3}\left(X^{\prime}\right)
$$
and on $G r_{2}^{W} H^{3}$

$$
H^{2}(E) \cap F^{2} H^{3} \subset H^{2}(E) \cap F^{1} H^{3} \subset H^{2}(E) \cap F^{0} H^{3}=H^{2}(E)
$$

lead to pure Hodge structures on $H^{3}\left(X^{\prime}\right)$ and $H^{2}(E)$. $H^{3}\left(X^{\prime}-E\right)$ forms a bundle $\mathcal{H}^{3}$ over the open-closed moduli space $\mathcal{M}$ with the Gauss-Manin connection $\nabla$ satisfying the Griffish transversality condition

$$
\nabla \mathcal{F}^{p} \in \mathcal{F}^{p-1} \otimes \Omega_{\mathcal{M}}^{1}
$$

The flatness of the Gauss-Manin connection leads to $\mathrm{N}=1$ special geometry and a PicardFuchs system of differential equations that govern the mirror maps and superpotentials.

The geometric setting we are interested in is a hypersurface $X: P=0$ with a curve $S$ on it, $S: P=0, h_{1}=h_{2}=0$. After blowing up along $S$, the blow-up manifold $X^{\prime}$ is given globally as the complete intersection in the total space of the projective bundle $\mathcal{W}=\mathbb{P}\left(\mathcal{O}\left(D_{1}\right) \oplus \mathcal{O}\left(D_{2}\right)\right)$,

$$
P=0, \quad Q \equiv y_{1} h_{2}-y_{2} h_{1}=0
$$

where $\left(y_{1}, y_{2}\right) \sim \lambda\left(y_{1}, y_{2}\right)$ is the projective coordinates on the $\mathbb{P}^{1}$-fiber of the blow-up $X^{\prime}$. We have to emphasize that $X^{\prime}$ is not Calabi-Yau since the first Chern class is nonzero. In addition, the blow-up procedure do not introduce new degrees of freedom associated to deformations of $E$. Under blowing up map, the open-closed moduli space of $(X, S)$ is mapped into the complex structure deformation of $X^{\prime}$. This enable us to calculate the superpotential $W_{\text {brane }}$ for B- branes wrapping rational curves via the periods on the complex structure moduli space of $X^{\prime}$ determined by Picard-Fuchs equations.

\section{Two closed and two open moduli case}

\subsection{Open-closed GKZ-system: branes on $X_{9}^{(1,1,1,3,3)}$}

\subsubsection{Five branes wrapping lines}

The Calabi-Yau threefold $X_{9}^{(1,1,1,3,3)}$ is defined as the mirror of the Calabi-Yau hypersurface $X^{*}$ in $\mathbb{P}_{(1,1,1,3,3)}^{4}$ with $h^{2,1}=2$ complex structure moduli and the charge vectors of the GLSM for the A model manifold are given by:

\begin{tabular}{cc|cccccc}
\hline & 0 & 1 & 2 & 3 & 4 & 5 & 6 \\
\hline$l^{1}$ & -3 & 0 & 0 & 0 & 1 & 1 & 1 \\
$l^{2}$ & 0 & 1 & 1 & 1 & 0 & 0 & -3 \\
\hline
\end{tabular}


The hypersurface constraint for the mirror manifold, written in homogeneous coordinates of $\mathbb{P}_{(1,1,1,3,3)}$, is

$$
P=x_{1}^{9}+x_{2}^{9}+x_{3}^{9}+x_{4}^{3}+x_{5}^{3}+\psi\left(x_{1} x_{2} x_{3} x_{4} x_{5}\right)+\phi\left(x_{1} x_{2} x_{3}\right)^{3}
$$

where $\psi=z_{1}^{-\frac{1}{3}} z_{2}^{-\frac{1}{9}}$ and $\phi=z_{2}^{-\frac{1}{3}}$. The Greene-Plesser orbifold group G acts as $x_{i} \rightarrow \lambda_{k}^{g_{k, i}} x_{i}$ with $\lambda_{1}^{9}=\lambda_{2}^{9}=1, \lambda_{3}^{3}=1$ and weights

$$
\mathbb{Z}_{9}: g_{1}=(1,-1,0,0,0), \quad \mathbb{Z}_{9}: g_{2}=(1,0,-1,0,0), \quad \mathbb{Z}_{3}: g_{3}=(0,0,0,1,-1)
$$

Next, we add a five-brane wrapping a rational curve on a toric curve $S$

$$
\begin{aligned}
S: & P & =0, & h_{1} \equiv \alpha^{3} \gamma^{3} x_{2}^{9}-\beta^{6}\left(x_{1} x_{2} x_{2}\right)^{3}=0, \quad h_{2} \equiv \beta^{3} \gamma^{3} x_{3}^{9}-\alpha^{6}\left(x_{1} x_{2} x_{3}\right)^{3}=0 \\
& \hat{l}^{1}=(0,0,1,0,0,0,-1), & \hat{l}^{2}=(0,0,0,1,0,0,-1) &
\end{aligned}
$$

An equivalent and convenient form is obtained after simple algebraic manipulations

$$
S: P=0, \quad \alpha^{9} x_{2}^{9}-\beta^{9} x_{3}^{9}=0, \quad \alpha^{9} x_{1}^{9}-\gamma^{9} x_{3}^{9}=0
$$

For generic values of the moduli in (3.2), $S$ is an irreducible high genus Riemann surface. But we can make a linearization by following steps: to begin with, we inserted $h_{1}$ and $h_{2}$ into $P$,

$$
\tilde{\mathbb{P}}^{1}: \quad \eta_{1} x_{4}+\sqrt[3]{x_{5}^{3}+m\left(x_{3}, x_{4}, x_{5}\right)}=0, \quad \eta_{2} \alpha x_{2}-\beta x_{3}=0, \quad \eta_{3} \alpha x_{1}-\gamma x_{3}=0
$$

Here $\eta_{1}^{3}=\eta_{2}^{9}=\eta_{3}^{9}=1$ and

$$
m\left(x_{3}, x_{4}, x_{5}\right)=\left(\frac{\alpha^{9}+\beta^{9}+\gamma^{9}}{\alpha^{9}}+\frac{\alpha^{3} \beta^{3} \gamma^{3}}{\alpha^{9}} \phi\right) x_{3}^{9}+\frac{\alpha \beta \gamma}{\alpha^{3}} \psi x_{3}^{3} x_{4} x_{5}
$$

Due to the non-trivial branching of the roots of unity, (3.3) is non-holomorphic, i.e. it is a non-holomorphic family of rational curves on $X$.

For special loci of $\mathcal{M}(S)$ where $m\left(x_{1}, x_{2}, x_{5}\right)$ vanishes identically,

$$
\mathcal{M}_{\mathbb{P}^{1}}(S): \quad \alpha^{9}+\beta^{9}+\gamma^{9}+\phi \alpha^{3} \beta^{3} \gamma^{3}=0, \quad \psi \alpha \beta \gamma=0
$$

the Riemann surface $S$ in (3.3) degenerates to

$$
S: \quad h_{0} \equiv x_{4}^{3}+x_{5}^{3}, \quad h_{1}=\alpha^{9} x_{2}^{9}-\beta^{9} x_{3}^{9}, \quad h_{2}=\alpha^{9} x_{1}^{9}-\gamma^{9} x_{3}^{9}
$$

Under the action of $G=\mathbb{Z}_{9}^{2} \times \mathbb{Z}_{3}$, (3.5) describes a single line,

$$
\mathbb{P}^{1}: \quad \eta_{1} x_{4}+x_{5}=0, \quad \alpha x_{2}-\beta x_{3}=0, \quad \alpha x_{1}-\gamma x_{3}=0
$$

In other words, these lines in $\mathbb{P}^{4}$ have a parametrization by homogeneous coordinates $U, V$ on $\mathbb{P}^{1}$ as the Veronese mapping

$$
\begin{aligned}
\mathcal{M}_{\mathbb{P}^{1}}(S) & \hookrightarrow \mathcal{M}(S) \\
(U, V) & \mapsto\left(\gamma U, \beta U, \alpha U,-\eta_{1} V, V\right), \quad \eta_{1}^{3}=1
\end{aligned}
$$




\begin{tabular}{|c|c|c|c|c|c|c|c|c|c|}
\hline & \multicolumn{4}{|c|}{$\Delta^{*}$} & \multicolumn{2}{|c|}{$\begin{array}{ll}l^{1} & l^{2}\end{array}$} & & \multicolumn{2}{|l|}{$\hat{l}^{1}$} \\
\hline$v_{0}^{*}$ & 0 & 0 & 0 & 0 & -3 & 0 & $x_{1} x_{2} x_{3} x_{4} x_{5}$ & 0 & 0 \\
\hline$v_{1}^{*}$ & -1 & -1 & -3 & -3 & 0 & 1 & $x_{1}^{9}$ & 0 & 0 \\
\hline$v_{2}^{*}$ & 1 & 0 & 0 & 0 & 0 & 1 & $x_{2}^{9}$ & 1 & 0 \\
\hline$v_{3}^{*}$ & 0 & 1 & 0 & 0 & 0 & 1 & $x_{3}^{9}$ & 0 & 1 \\
\hline$v_{4}^{*}$ & 0 & 0 & 1 & 0 & 1 & 0 & $x_{4}^{3}$ & 0 & 0 \\
\hline$v_{5}^{*}$ & 0 & 0 & 0 & 1 & 1 & 0 & $x_{5}^{3}$ & 0 & 0 \\
\hline$v_{6}^{*}$ & 0 & 0 & -1 & -1 & 1 & -3 & $\left(x_{1} x_{2} x_{3}\right)^{3}$ & -1 & -1 \\
\hline
\end{tabular}

Table 1. Toric Data of A-model side.

Thus all obstructed deformations locate at $\mathcal{M}(S)-\mathcal{M}_{\mathbb{P}^{1}}(S)$, inducing a non-trivial superpotential, which plays an important role in research on obstruction deformation, especially for a manifold with a submanifold on it. As we know, blowing up is very effective method to handle such case. According to (2.15), we construct the blow-up manifold $X^{\prime}$ given by the complete intersection in projective bundle

$$
X^{\prime}: P=0, \quad Q=y_{1} h_{2}-y_{2} h_{1}
$$

It is obvious from above defining equations that the moduli of $S$ described by the coefficients of the monomials in $h_{i}, i=1,2$ turn into complex structure moduli of $X^{\prime}$. We obtain the embedding of the obstructed deformation space of $\left(X, \tilde{\mathbb{P}}^{1}\right)$ into the complex structure moduli space of $X^{\prime}$, which is crucial for the following superpotential calculations.

\subsubsection{Toric branes and blowing up geometry}

Now, we study the A-model manifold, whose toric polyhedron is denoted by $\Delta^{*}$ and charge vectors are denoted by $l^{1}$ and $l^{2}$. The integral vertices of polyhedron $\Delta^{*}$ and the charge vectors $l^{1}, l^{2}$ for A-model manifold, $\hat{l}^{1}, \hat{l}^{2}$ for A-branes is as table 1 .

From above toric data of $\Delta^{*}$ and its dual polyhedron $\Delta$,

$$
\begin{array}{ll}
v_{1}=(-1,-1,-1,-1), & v_{2}=(8,-1,-1,-1), \quad v_{3}=(-1,8,-1,-1), \\
v_{4}=(-1,-1,2,-1), & v_{5}=(-1,-1,-1,2)
\end{array}
$$

In B-model, the defining equations of the mirror manifold $X$ and the curve $S$ as follow in torus coordinates

$$
\begin{aligned}
X: & P=a_{0}+a_{1}\left(X_{2} X_{3} X_{4}^{3} X_{5}^{3}\right)^{-1}+a_{2} X_{2}+a_{3} X_{3}+a_{4} X_{4}+a_{5} X_{5}+a_{6}\left(X_{4} X_{5}\right)^{-1} \\
S: & h_{1}=a_{7} X_{2}+a_{8}\left(X_{4} X_{5}\right)^{-1}, \quad h_{2}=a_{9} X_{3}+a_{10}\left(X_{4} X_{5}\right)^{-1}
\end{aligned}
$$

where $a_{i}$ 's are free complex-valued coefficients. With the abbreviation of logarithmic deriva- 
tives $\vartheta_{i}=a_{i} \frac{\partial}{\partial a_{i}}$, the GKZ-system of $X$ by $(2.7)$ is,

$$
\begin{array}{rlrl}
\mathcal{Z}_{0} & =\sum_{i=0}^{6} \vartheta_{i}+1, & \mathcal{Z}_{i}=-\vartheta_{1}+\vartheta_{i+1}, & i=1,2, \\
\mathcal{Z}_{i}=-3 \vartheta_{1}+\vartheta_{i+1}-\vartheta_{6}, & & i=3,4 \\
\mathcal{L}_{1}=\prod_{i=4}^{6} \frac{\partial}{\partial a_{i}}-\left(\frac{\partial}{\partial a_{0}}\right)^{3}, & \mathcal{L}_{2}=\prod_{i=1}^{3} \frac{\partial}{\partial a_{i}}-\left(\frac{\partial}{\partial a_{6}}\right)^{3} &
\end{array}
$$

Here $\mathcal{Z}_{0}$ represent the invariance of $P$ under overall rescaling and other $\mathcal{Z}_{i}$ 's relate to the invariance of $P$ under the rescaling of torus coordinates $X_{i}$ 's combined with the rescaling of coefficients $a_{i}$ 's.

$$
\begin{aligned}
\mathcal{Z}_{i}: & X_{i+1} & \mapsto \lambda X_{i+1}, & \left(a_{1}, a_{i+1}\right) & \mapsto\left(\lambda a_{1}, \lambda^{-1} a_{i+1}\right), & i=1,2 \\
\mathcal{Z}_{i}: & X_{i+1} & \mapsto \lambda X_{i+1}, & \left(a_{1}, a_{i+1}, a_{6}\right) & \mapsto\left(\lambda^{3} a_{1}, \lambda^{-1} a_{i+1}, \lambda a_{6}\right), & i=3,4
\end{aligned}
$$

Operators $\mathcal{L}_{i}$ 's relate to the symmetries among the Laurent monomials in $P(3.7)$,

$$
\begin{array}{rlrl}
\mathcal{L}_{1}: & X_{4} X_{5}\left(X_{4} X_{5}\right)^{-1} & =1 \\
\mathcal{L}_{2}: & \left(X_{2}^{-1} X_{3}^{-1} X_{4}^{-3} X_{5}^{-3}\right) X_{2} X_{3}=\left(\left(X_{4} X_{5}\right)^{-1}\right)^{3}
\end{array}
$$

By blowing up $X$ along $S$, the blow-up manifold $X^{\prime}$ is obtained.

$$
X^{\prime}: P=0, \quad Q=y_{1}\left(a_{9} X_{3}+a_{10}\left(X_{4} X_{5}\right)^{-1}\right)-y_{2}\left(a_{7} X_{2}+a_{8}\left(X_{4} X_{5}\right)^{-1}\right)
$$

After careful observation on the torus symmetry of $X^{\prime}$, we can obtain the infinitesimal generators which are belong to GKZ system associated to $X^{\prime}$,

$$
\begin{aligned}
\mathcal{Z}_{0}^{\prime} & =\sum_{i=0}^{6} \vartheta_{i}+1, \quad \mathcal{Z}_{1}^{\prime}=\sum_{i=7}^{10} \vartheta_{i}, \quad \mathcal{Z}_{2}^{\prime}=-\vartheta_{1}+\vartheta_{2}+\vartheta_{7}, \quad \mathcal{Z}_{3}^{\prime}=-\vartheta_{1}+\vartheta_{3}+\vartheta_{9}, \\
\mathcal{Z}_{i}^{\prime} & =-3 \vartheta_{1}+\vartheta_{i}-\vartheta_{6}-\vartheta_{8}-\vartheta_{10} \quad i=4,5, \quad \mathcal{Z}_{6}^{\prime}=-\vartheta_{7}-\vartheta_{8}+\vartheta_{9}+\vartheta_{10} \\
\mathcal{L}_{1}^{\prime} & =\prod_{i=4}^{6} \frac{\partial}{\partial a_{i}}-\left(\frac{\partial}{\partial a_{0}}\right)^{3}, \quad \mathcal{L}_{2}^{\prime}=\prod_{i=1}^{3} \frac{\partial}{\partial a_{i}}-\left(\frac{\partial}{\partial a_{6}}\right)^{3} \\
\mathcal{L}_{3}^{\prime} & =\frac{\partial}{\partial a_{6}} \frac{\partial}{\partial a_{7}}-\frac{\partial}{\partial a_{2}} \frac{\partial}{\partial a_{8}}, \quad \mathcal{L}_{4}^{\prime}=\frac{\partial}{\partial a_{3}} \frac{\partial}{\partial a_{10}}-\frac{\partial}{\partial a_{6}} \frac{\partial}{\partial a_{9}}
\end{aligned}
$$

Here $\mathcal{Z}_{0}, \mathcal{Z}_{1}$ are associated with the overall rescaling with respect to $P=0, Q=0$ respectively. $\mathcal{Z}_{i}, i=2, \ldots 5$ are related to the torus symmetry as before.

$$
\begin{aligned}
\mathcal{Z}_{2}^{\prime}: & X_{2} & \mapsto \lambda X_{2}, & \left(a_{1}, a_{2}, a_{7}\right) & \mapsto\left(\lambda a_{1}, \lambda^{-1} a_{2}, \lambda^{-1} a_{7}\right) \\
\mathcal{Z}_{3}^{\prime}: & X_{3} & \mapsto \lambda X_{3}, & \left(a_{1}, a_{3}, a_{9}\right) & \mapsto\left(\lambda a_{1}, \lambda^{-1} a_{3}, \lambda^{-1} a_{9}\right) \\
\mathcal{Z}_{4}^{\prime}: & X_{4} & \mapsto \lambda X_{4}, & \left(a_{1}, a_{4}, a_{6}, a_{8}, a_{10}\right) & \mapsto\left(\lambda^{3} a_{1}, \lambda^{-1} a_{4}, \lambda a_{6}, \lambda a_{8}, \lambda a_{10}\right) \\
\mathcal{Z}_{5}^{\prime}: & X_{5} & \mapsto \lambda X_{5}, & \left(a_{1}, a_{5}, a_{6}, a_{8}, a_{10}\right) & \mapsto\left(\lambda^{3} a_{1}, \lambda^{-1} a_{5}, \lambda a_{6}, \lambda a_{8}, \lambda a_{10}\right)
\end{aligned}
$$

In addition, $\mathcal{Z}_{6}^{\prime}$ is related to the torus symmetry $\left(y_{1}, y_{2}\right) \mapsto\left(\lambda y_{1}, \lambda^{-1} y_{2}\right)$. The new $\mathcal{L}_{3}, \mathcal{L}_{4}$ incorporate the parameter $a_{7}, \ldots, a_{10}$ that are associated with the open-closed moduli of 
the curve $S$. All these GKZ operators annihilate the holomorphic three form $\Omega^{\prime}$ on $X^{\prime}$ that is the pull back of the homomorphic three form $\Omega$ on $X$, i.e. $\Omega^{\prime}=\pi^{*} \Omega$.

Now, we formulate the GKZ-system (3.10) on an enhanced polyhedron $\Delta^{\prime}$, by adding additional vertices on the original polyhedron $\Delta^{*}$.

\begin{tabular}{|c|c|c|c|c|c|c|c|c|c|c|c|c|}
\hline & \multicolumn{7}{|c|}{$\Delta^{\prime}$} & $l_{1}^{\prime}$ & $l_{2}^{\prime}$ & $l_{3}^{\prime}$ & $l_{4}^{\prime}$ & \\
\hline$v_{0}^{\prime}$ & 1 & 0 & 0 & 0 & 0 & 0 & 0 & -3 & 0 & 0 & 0 & $w_{0}^{\prime}=x_{1} x_{2} x_{3} x_{4} x_{5}$ \\
\hline$v_{1}^{\prime}$ & 1 & 0 & -1 & -1 & -3 & -3 & 0 & 0 & 1 & 0 & 0 & $w_{1}^{\prime}=x_{1}^{9}$ \\
\hline$v_{2}^{\prime}$ & 1 & 0 & 1 & 0 & 0 & 0 & 0 & 1 & 1 & -1 & 0 & $w_{2}^{\prime}=x_{2}^{9}$ \\
\hline$v_{3}^{\prime}$ & 1 & 0 & 0 & 1 & 0 & 0 & 0 & 0 & 0 & 0 & 1 & $w_{3}^{\prime}=x_{3}^{9}$ \\
\hline$v_{4}^{\prime}$ & 1 & 0 & 0 & 0 & 1 & 0 & 0 & 1 & 0 & 0 & 0 & $w_{4}^{\prime}=x_{4}^{3}$ \\
\hline$v_{5}^{\prime}$ & 1 & 0 & 0 & 0 & 0 & 1 & 0 & 1 & 0 & 0 & 0 & $w_{5}^{\prime}=x_{5}^{3}$ \\
\hline$v_{6}^{\prime}$ & 1 & 0 & 0 & 0 & -1 & -1 & 0 & 0 & -2 & 1 & -1 & $w_{6}^{\prime}=\left(x_{1} x_{2} x_{3}\right)^{3}$ \\
\hline$v_{7}^{\prime}$ & 0 & 1 & 1 & 0 & 0 & 0 & -1 & -1 & 0 & 1 & 0 & $w_{7}^{\prime}=y_{1} w_{2}^{\prime}$ \\
\hline$v_{8}^{\prime}$ & 0 & 1 & 0 & 0 & -1 & -1 & -1 & 1 & 0 & -1 & 0 & $w_{8}^{\prime}=y_{1} w_{6}^{\prime}$ \\
\hline$v_{9}^{\prime}$ & 0 & 1 & 0 & 1 & 0 & 0 & 1 & 0 & 1 & 0 & -1 & $w_{9}^{\prime}=y_{2} w_{3}^{\prime}$ \\
\hline$v_{10}^{\prime}$ & 0 & 1 & 0 & 0 & -1 & -1 & 1 & 0 & -1 & 0 & 1 & $w_{10}^{\prime}=y_{2} w_{6}^{\prime}$ \\
\hline
\end{tabular}

where $v_{i}^{\prime}$ 's are the integral vertices of $\Delta^{\prime}$ and their corresponding monomials in homogeneous coordinates of $\mathbb{P}^{4}$ are $w_{i}^{\prime}$. The A-model closed string charge vectors and A-branes charge vectors relate to the maximal triangulation of $\Delta^{\prime}$ and satisfy the relations,

$$
l^{1}=l_{1}^{\prime}+l_{3}^{\prime}, l^{2}=l_{2}^{\prime}+l_{4}^{\prime}, \hat{l}^{1}=l_{3}^{\prime}, \hat{l}^{2}=l_{4}^{\prime}
$$

The coordinates $z_{j}$ by (2.7) on the complex structure moduli space of $X^{\prime}$.

$$
z_{1}=\frac{a_{2} a_{4} a_{5} a_{8}}{a_{0}^{3} a_{7}}, \quad z_{2}=\frac{a_{1} a_{2} a_{9}}{a_{6}^{2} a_{10}}, \quad z_{3}=\frac{a_{6} a_{7}}{a_{2} a_{8}}, \quad z_{4}=\frac{a_{3} a_{10}}{a_{6} a_{9}}
$$

Next, we convert the $\mathcal{L}_{i}$ operators to Picard-Fuchs operators $\mathcal{D}_{i}$, from differential equations about $a_{j}(j=0, \ldots, 10)$ to those about $z_{j}(j=1, \ldots, 4)$ of $X^{b}$. From table (3.11), we obtain the identity

$$
\begin{array}{llll}
\vartheta_{0}=-3 \theta_{1}, & \vartheta_{1}=\theta_{2}, & \vartheta_{2}=\theta_{1}+\theta_{2}-\theta_{3}, & \vartheta_{3}=\theta_{4}, \\
\vartheta_{4}=\theta_{1}, & \vartheta_{5}=\theta_{1}, & \vartheta_{6}=-2 \theta_{2}+\theta_{3}-\theta_{4}, & \\
\vartheta_{7}=-\theta_{1}+\theta_{3}, & \vartheta_{8}=\theta_{1}-\theta_{3}, & \vartheta_{9}=\theta_{2}-\theta_{4}, & \vartheta_{10}=\theta_{2}+\theta_{10}
\end{array}
$$

Inserting above relations between the logarithmic derivatives $\vartheta_{j}$ w.r.t. $a_{j}$ and the logarithmic derivatives $\theta_{j}$ w.r.t. $z_{j}$ into $\mathcal{L}$ operators in (3.10), the full set of Picard-Fuchs operators are obtained

$$
\begin{aligned}
& \mathcal{D}_{1}=\left(\theta_{1}+\theta_{2}-\theta_{3}\right) \theta_{1}^{2}\left(\theta_{1}-\theta_{3}\right)-z_{1}\left(-\theta_{1}+\theta_{3}\right) \prod_{i=1}^{3}\left(-3 \theta_{1}-i\right), \\
& \mathcal{D}_{2}=\theta_{2}\left(\theta_{1}+\theta_{2}-\theta_{3}\right)\left(\theta_{2}-\theta_{4}\right)-z_{2}\left(-2 \theta_{1}+\theta_{3}-\theta_{4}\right)\left(-\theta_{2}+\theta_{4}\right) \\
& \mathcal{D}_{3}=\left(-2 \theta_{1}+\theta_{3}-\theta_{4}\right)\left(-\theta_{1}+\theta_{3}\right)-z_{3}\left(\theta_{1}+\theta_{2}-\theta_{3}\right)\left(\theta_{1}-\theta_{3}\right), \\
& \mathcal{D}_{4}=\theta_{4}\left(-\theta_{2}+\theta_{4}\right)-z_{4}\left(-2 \theta_{1}+\theta_{3}-\theta_{4}\right)\left(\theta_{2}-\theta_{4}\right)
\end{aligned}
$$


where $\theta_{i}=z_{i} \frac{\partial}{\partial z_{i}}$ 's are the logarithmic derivatives and each operator $\mathcal{D}_{a}$ corresponds to a linear combination of the charge vectors among $l_{1}^{\prime}, l_{2}^{\prime}, l_{3}^{\prime}, l_{4}^{\prime}$.

\subsubsection{Brane superpotential and disk instantons}

Now, we solve the Picard-Fuchs equations (3.13) at $z_{i} \rightarrow 0$ and identified the mirror maps and superpotentials. By the method introduced in section 2.1, the fundamental period of $X$ as power series solution as follow

$$
\omega_{0}=1+6 z_{1} z_{3}+90 z_{1}^{2} z_{3}^{2}+1680 z_{1}^{3} z_{3}^{3}+34650 z_{1}^{4} z_{3}^{4}+10080 z_{1}^{3} z_{2} z_{3}^{3} z_{4}+\mathcal{O}\left(z^{8}\right) .
$$

There are four logarithmic solutions with leading term $\omega_{0} \log \left(Z_{i}\right)$

$$
\begin{aligned}
\omega_{1,1}= & \omega_{0} \log \left(z_{1}\right)-6 z_{1}+45 z_{1}^{2}-560 z_{1}^{3}+\frac{17325}{2} z_{1}^{4}-\frac{756756}{5} z_{1}^{5}+21 z_{1} z_{3}-180 z_{1}^{2} z_{3}+2520 z_{1}^{3} z_{3} \\
& -46200 z_{1}^{4} z_{3}+\frac{783}{2} z_{1}^{2} z_{3}^{2}-5040 z_{1}^{3} z_{3}^{2}+\mathcal{O}\left(z^{5}\right) \\
\omega_{1,2}= & \omega_{0} \log \left(z_{2}\right)+12 z_{1} z_{3}+270 z_{1}^{2} z_{3}^{2}-180 z_{1}^{2} z_{2} z_{3}^{2}-4 z_{2} z_{4}+6 z_{1} z_{3} z_{4}+12 z_{1} z_{2} z_{3} z_{4}+180 z_{1}^{2} z_{3}^{2} z_{4} \\
& +30 z_{2}^{2} z_{4}^{2}+\mathcal{O}\left(z^{5}\right) \\
\omega_{1,3}= & \omega_{0} \log \left(z_{3}\right)+6 z_{1}-45 z_{1}^{2}+560 z_{1}^{3}-\frac{17325}{2} z_{1}^{4}+\frac{756756}{5} z_{1}^{5}-6 z_{1} z_{3}+180 z_{1}^{2} z_{3}-2520 z_{1}^{3} z_{3} \\
& +46200 z_{1}^{4} z_{3}-135 z_{1}^{2} z_{3}^{2}+5040 z_{1}^{3} z_{3}^{2}+2 z_{2} z_{4}-6 z_{1} z_{2} z_{3} z_{4}-15 z_{2}^{2} z_{4}^{2}+\mathcal{O}\left(z^{5}\right) \\
\omega_{1,4}= & \omega_{0} \log \left(z_{4}\right) 6 z_{1} z_{3}+135 z_{1}^{2} z_{3}^{2}+180 z_{1}^{2} z_{2} z_{3}^{2}-2 z_{2} z_{4}-6 z_{1} z_{3} z_{4}+6 z_{1} z_{2} z_{3} z_{4}-180 z_{1}^{2} z_{3}^{2} z_{4} \\
& +15 z_{2}^{2} z_{4}^{2}+\mathcal{O}\left(z^{5}\right) .
\end{aligned}
$$

By the definition of the flat coordinates and mirror maps from Kahler moduli space to complex structure moduli space

$$
\begin{aligned}
t_{j} & =\frac{\omega_{1, j}}{\omega_{0}} \\
q_{j} & =e^{2 \pi i t_{j}}
\end{aligned}
$$

we obtain the $z_{j}$ as a series of $q_{j}$ upon inversion of the mirror maps

$$
\begin{aligned}
z_{1}= & q_{1}+6 q_{1}^{2}+9 q_{1}^{3}+56 q_{1}^{4}-300 q_{1}^{5}-21 q_{1}^{2} q_{3}-108 q_{1}^{3} q_{3}-225 q_{1}^{4} q_{3}+270 q_{1}^{3} q_{3}^{2} \\
& +42 q_{1}^{2} q_{2} q_{3} q_{4}+\mathcal{O}\left(q^{5}\right) \\
z_{2}= & q_{2}-12 q_{1} q_{2} q_{3}+54 q_{1}^{2} q_{2} q_{3}^{2}+4 q_{2}^{2} q_{4}-6 q_{1} q_{2} q_{3} q_{4}-132 q_{1} q_{2}^{2} q_{3} q_{4}+2 q_{2}^{3} q_{4}^{2}+\mathcal{O}\left(q^{5}\right) \\
z_{3}= & q_{3}-6 q_{1} q_{3}+27 q_{1}^{2} q_{3}-164 q_{1}^{3} q_{3}+1377 q_{1}^{4} q_{3}+6 q_{1} q_{3}^{2}-54 q_{1}^{2} q_{3}^{2}+414 q_{1}^{3} q_{3}^{2}+27 q_{1}^{2} q_{3}^{3} \\
& -2 q_{2} q_{3} q_{4}+12 q_{1} q_{2} q_{3} q_{4}-54 q_{1}^{2} q_{2} q_{3} q_{4}+30 q_{1} q_{2} q_{3}^{2} q_{4}+\mathcal{O}\left(q^{5}\right) \\
z_{4}= & q_{4}-6 q_{1} q_{3} q_{4}+9 q_{1}^{2} q_{3}^{2} q_{4}+2 q_{2} q_{4}^{2}+6 q_{1} q_{3} q_{4}^{2}-54 q_{1} q_{2} q_{3} q_{4}^{2}-q_{2}^{2} q_{4}^{3}+\mathcal{O}\left(q^{5}\right)
\end{aligned}
$$

In addition, we abbreviate the double logarithmic solutions by their leading terms

$$
\begin{aligned}
& \frac{1}{2} \ell_{1}^{2}+2 \ell_{3} \ell_{4}, \quad \frac{1}{2} \ell_{2}^{2}, \quad \frac{1}{2} \ell_{3}^{2}+\ell_{3} \ell_{4}, \quad \frac{1}{2} \ell_{4}^{2}, \\
& \ell_{1} \ell_{2}+\ell_{2} \ell_{4}, \quad \ell_{1} \ell_{3}-3 \ell_{3} \ell_{4}, \quad \ell_{1} \ell_{4}+\ell_{3} \ell_{4}, \quad \ell_{2} \ell_{3}
\end{aligned}
$$


where $\log \left(z_{i}\right)$ 's are abbreviated as $\ell_{i}$ 's. According to above, a specific linear combination of double logarithmic solutions is constructed,

$$
\mathcal{W}_{\text {brane }}=3 t_{1} t_{2}-3 t_{3} t_{4}+\sum_{N} N_{d_{1}, d_{2}, d_{3}, d_{4}} \operatorname{Li}_{2}\left(q_{1}^{d_{1}} q_{2}^{d_{2}} q_{3}^{d_{3}} q_{4}^{d_{4}}\right)
$$

where $\operatorname{Li}_{2}\left(q_{1}^{d_{1}} q_{2}^{d_{2}} q_{3}^{d_{3}} q_{4}^{d_{4}}\right)=\sum_{n=1}^{\infty} \frac{q_{1}^{n d_{1}} q_{2}^{n d_{2}} q_{3}^{n d_{3}} q_{4}^{n d_{4}}}{n^{2}}$ is the dilogarithm function. We extract the disk instantons $N_{d_{1}, d_{2}, d_{3}, d_{4}}$ from $\mathcal{W}_{\text {brane }}$ and present first a few invariants of the form $N_{k, m, k, m+n}$ in table 8. When focusing only on the invariants of the form $N_{k, m, k, m+n}$,

$$
\mathcal{W}_{\text {brane }}=\cdots+\sum_{N} N_{k, m+n, k, n} \operatorname{Li}_{2}\left(\left(\tilde{q_{1}}\right)^{k}\left(\tilde{q_{2}}\right)^{m}{\tilde{q_{3}}}^{n}\right)
$$

where $\ldots$ are terms independent of invariants, and $\tilde{q_{1}}=q_{1} q_{3}, \tilde{q_{2}}=q_{2} q_{4}, \tilde{q_{3}}=q_{4}$. The superpotential (3.17) can be written as

$$
\mathcal{W}_{\text {brane }}=\cdots+\sum_{N} N_{a, b, c} \operatorname{Li}_{2}\left(\left(\widetilde{q_{1}}\right)^{a}\left(\tilde{q_{2}}\right)^{b} \tilde{q}_{c}^{c}\right)
$$

which are essentially the superpotential of the model in [8]. Therefore, our invariants at first several order exactly match with the data of table 5 in [8] and these invariants are marked by blue color in table 8 . In addition, we also calculate the invariants at higher order and put them into table 8 .

\subsubsection{Another curve on $\mathrm{X}$}

Following the similar logic, we consider another toric curve $S$ on $\mathrm{X}$ with charge vectors

$$
\begin{aligned}
P & =0, & & \\
h_{1} & \equiv a_{7}\left(x_{1} x_{2} x_{3} x_{4} x_{5}\right)+a_{8} x_{4}^{3}=0, & & \hat{l}_{1}=(-1,0,0,0,1,0,0) \\
h_{2} & \equiv a_{9} x_{3}^{9}+a_{10} x_{4}^{3}=0, & & \hat{l}_{2}=(0,0,0,1,-1,0,0)
\end{aligned}
$$

After blowing up, the toric data of mirror manifold of $X^{\prime}$ is determined by charge vectors

$$
\begin{array}{ll}
l_{1}^{\prime}=(-2,0,0,0,0,1,1,-1,1,0,0), & l_{2}^{\prime}=(0,1,1,0,1,0,-3,0,0,1,-1), \\
l_{3}^{\prime}=(-1,0,0,0,1,0,0,1,-1,0,0), & l_{4}^{\prime}=(0,0,0,1,-1,0,0,0,0,-1,1)
\end{array}
$$

satisfying $l^{1}=l_{1}^{\prime}+l_{3}^{\prime}, l^{2}=l_{2}^{\prime}+l_{4}^{\prime}$, from which we obtain the local coordinates on complex structure moduli of $X^{\prime}$

$$
z_{1}=\frac{a_{5} a_{6} a_{8}}{a_{0}^{2} a_{7}}, \quad z_{2}=\frac{a_{1} a_{2} a_{4} a_{9}}{a_{6}^{3} a_{10}}, \quad z_{3}=\frac{a_{4} a_{7}}{a_{0} a_{8}}, \quad z_{4}=\frac{a_{6} a_{10}}{a_{4} a_{9}}
$$

and Picard-Fuchs operators

$$
\begin{aligned}
& \mathcal{D}_{1}=\theta_{1}\left(\theta_{1}-3 \theta_{2}\right)\left(\theta_{1}-\theta_{3}\right)-z_{1}\left(-\theta_{1}+\theta_{3}\right) \prod_{i=1}^{2}\left(-2 \theta_{1}-\theta_{3}-i\right) \\
& \mathcal{D}_{2}=\left(\theta_{2}+\theta_{3}-\theta_{4}\right)\left(\theta_{2}\right)^{2}\left(\theta_{2}-\theta_{4}\right)-z_{2}\left(\theta_{1}-3 \theta_{2}\right)^{3}\left(-\theta_{2}-\theta_{4}\right) \\
& \mathcal{D}_{3}=\left(\theta_{2}+\theta_{3}-\theta_{4}\right)\left(-\theta_{1}+\theta_{3}\right)-z_{3}\left(-2 \theta_{1}-\theta_{3}-1\right)\left(\theta_{1}-\theta_{3}\right) \\
& \mathcal{D}_{4}=\theta_{4}\left(-\theta_{2}+\theta_{4}\right)-z_{4}\left(\theta_{2}+\theta_{3}-\theta_{4}\right)\left(\theta_{2}-\theta_{4}\right)
\end{aligned}
$$


As before, we find single logarithmic solutions

$$
\begin{aligned}
\omega_{1,1}= & \omega_{0} \log \left(z_{1}\right)-2 z_{1}+3 z_{1}^{2}-\frac{20}{3} z_{1}^{3}+\frac{35}{2} z_{1}^{4}-\frac{252}{5} z_{1}^{5}+z_{3}+10 z_{1} z_{3}-30 z_{1}^{2} z_{3}+70 z_{1}^{3} z_{3} \\
& -210 z_{1}^{4} z_{3}-\frac{1}{2} z 3^{2}+12 z_{1} z_{3}^{2}+171 z_{1}^{2} z_{3}^{2}-560 z_{1}^{3} z_{3}^{2}+\frac{1}{3} z_{3}^{3}-10 z_{1} z_{3}^{3}+210 z_{1}^{2} z_{3}^{3}-\frac{1}{4} z_{3}^{4} \\
& +10 z_{1} z_{3}^{4}+\frac{1}{5} z_{3}^{5}+2 z_{2} z_{4}-40 z_{1}^{3} z_{2} z_{4}-6 z_{1} z_{2} z_{3} z_{4}+\mathcal{O}\left(z^{5}\right) \\
\omega_{1,2}= & \omega_{0} \log \left(z_{2}\right)+12 z_{1} z_{3}+270 z_{1}^{2} z_{3}^{2}-6 z_{2} z_{4}+6 z_{1} z_{3} z_{4}+18 z_{1} z_{2} z_{3} z_{4}+180 z_{1}^{2} z_{3}^{2} z_{4} \\
& +45 z_{2}^{2} z_{4}^{2}+\mathcal{O}\left(z^{5}\right) \\
\omega_{1,3}= & \omega_{0} \log \left(z_{3}\right)+2 z_{1}-3 z_{1}^{2}+\frac{20}{3} z_{1}^{3}-\frac{35}{2} z_{1}^{4}+\frac{252}{5} z_{1}^{5}-z_{3}+5 z_{1} z_{3}+30 z_{1}^{2} z_{3}-70 z_{1}^{3} z_{3} \\
& +210 z_{1}^{4} z_{3}+\frac{1}{2} z_{3}^{2}-12 z_{1} z_{3}^{2}+\frac{171}{2} z_{1}^{2} z_{3}^{2}+560 z_{1}^{3} z_{3}^{2}-\frac{1}{3} z_{3}^{3}+10 z_{1} z_{3}^{3}-210 z_{1}^{2} z_{3}^{3}+\frac{1}{4} z_{3}^{4} \\
& -10 z_{1} z_{3}^{4}-\frac{1}{5} z_{3}^{5}+40 z_{1}^{3} z_{2} z_{4}+\mathcal{O}\left(z^{5}\right) \\
\omega_{1,4}= & \omega_{0} \log \left(z_{4}\right)+6 z_{1} z_{3}+135 z_{1}^{2} z_{3}^{2}-6 z_{1} z_{3} z_{4}-180 z_{1}^{2} z_{3}^{2} z_{4}+\mathcal{O}\left(z^{5}\right)
\end{aligned}
$$

and mirror maps

$$
\begin{aligned}
z_{1}= & q_{1}+2 q_{1}^{2}+3 q_{1}^{3}+4 q_{1}^{4}+5 q_{1}^{5}-q_{1} q_{3}-12 q_{1}^{2} q_{3}-24 q_{1}^{3} q_{3}-36 q_{1}^{4} q_{3}+9 q_{1}^{2} q_{3}^{2}+108 q_{1}^{3} q_{3}^{2}+q_{1}^{2} q_{3}^{3} \\
& -2 q_{1} q_{2} q_{4}-8 q_{1}^{2} q_{2} q_{4}-18 q_{1}^{3} q_{2} q_{4}+2 q_{1} q_{2} q_{3} q_{4}+102 q_{1}^{2} q_{2} q_{3} q_{4}+\mathcal{O}\left(q^{5}\right) \\
z_{2}= & q_{2}-12 q_{1} q_{2} q_{3}+54 q_{1}^{2} q_{2} q_{3}^{2}+6 q_{2}^{2} q_{4}-6 q_{1} q_{2} q_{3} q_{4}-210 q_{1} q_{2}^{2} q_{3} q_{4}+9 q_{2}^{3} q_{4}^{2}+\mathcal{O}\left(q^{5}\right) \\
z_{3}= & q_{3}-2 q_{1} q_{3}+q_{1}^{2} q_{3}+q_{3}^{2}-7 q_{1} q_{3}^{2}+12 q_{1}^{2} q_{3}^{2}-7 q_{1}^{3} q_{3}^{2}+q_{1}^{4} q_{3}^{2}+q_{3}^{3}-6 q_{1} q_{3}^{3}+42 q_{1}^{2} q_{3}^{3}+q_{3}^{4} \\
& -6 q_{1} q_{3}^{4}+q_{3}^{5}+4 q_{1} q_{2} q_{3} q_{4}-4 q_{1}^{2} q_{2} q_{3} q_{4}+14 q_{1} q_{2} z_{3}^{2} q_{4}+\mathcal{O}\left(q^{5}\right) \\
z_{4}= & q_{4}-6 q_{1} q_{3} q_{4}+9 q_{1}^{2} q_{3}^{2} q_{4}+6 q_{1} q_{3} q_{4}^{2}+12 q_{1} q_{2} q_{3} q_{4}^{2}+\mathcal{O}\left(q^{5}\right)
\end{aligned}
$$

as single logarithmic solutions.

The superpotentials is constructed as follow

$$
\mathcal{W}_{\text {brane }}=9 t_{1}^{2}+6 t_{1} t_{2}+6 t_{1} t_{3}+\sum_{N} N_{d_{1}, d_{2}, d_{3}, d_{4}} \operatorname{Li}_{2}\left(q_{1}^{d_{1}} q_{2}^{d_{2}} q_{3}^{d_{3}} q_{4}^{d_{4}}\right)
$$

as linear combination of double logarithmic solutions. We extract and summarize the Ooguri-Vafa invariants of the form $N_{k, m, k, m+n}$ and $N_{k, m+n, k, n}$ in table 9 and table 10. At first several order, our result marked by blue color exactly agree with table 6 in [8] and we also present higher order result in the table.

\subsection{Open-closed GKZ-system: branes on $X_{8}^{(1,1,2,2,2)}$}

\subsubsection{Branes wrapping rational curves and blowing up geometry}

Now, we study the A-model manifold, whose toric polyhedron is denoted by $\Delta^{*}$ and charge vectors are denoted by $l^{1}$ and $l^{2}$. A-model manifold and A-brane are specified by the following toric data. The hypersurface equation for $X$, written in homogeneous coordinates of $\mathbb{P}_{(1,1,2,2,2)}$, is

$$
P=x_{1}^{8}+x_{2}^{8}+x_{3}^{4}+x_{4}^{4}+x_{5}^{4}+\psi\left(x_{1} x_{2} x_{3} x_{4} x_{5}\right)+\phi\left(x_{1} x_{2}\right)^{4}
$$




\begin{tabular}{|c|c|c|c|c|c|c|c|c|c|}
\hline & \multicolumn{4}{|c|}{$\Delta^{*}$} & \multicolumn{2}{|c|}{$l^{1} \quad l^{2}$} & \multirow[b]{2}{*}{$x_{1} x_{2} x_{3} x_{4} x_{5}$} & \multirow{2}{*}{$\begin{array}{l}\hat{l}^{1} \\
-1\end{array}$} & $\hat{l}^{2}$ \\
\hline$v_{0}^{*}$ & 0 & 0 & 0 & 0 & -4 & 0 & & & 0 \\
\hline$v_{1}^{*}$ & -1 & -2 & -2 & -2 & 0 & 1 & $x_{1}^{8}$ & 0 & -1 \\
\hline$v_{2}^{*}$ & 1 & 0 & 0 & 0 & 0 & 1 & $x_{2}^{8}$ & 0 & 1 \\
\hline$v_{3}^{*}$ & 0 & 1 & 0 & 0 & 1 & 0 & $x_{3}^{4}$ & 1 & 0 \\
\hline$v_{4}^{*}$ & 0 & 0 & 1 & 0 & 1 & 0 & $x_{4}^{4}$ & 0 & 0 \\
\hline$v_{5}^{*}$ & 0 & 0 & 0 & 1 & 1 & 0 & $x_{5}^{4}$ & 0 & 0 \\
\hline$v_{6}^{*}$ & 0 & -1 & -1 & -1 & 1 & -2 & $\left(x_{1} x_{2}\right)^{4}$ & 0 & 0 \\
\hline
\end{tabular}

Table 2. Toric Data of A-model side.

where $\psi=z_{1}^{-\frac{1}{4}} z_{2}^{-\frac{1}{8}}$ and $\phi=z_{2}^{-\frac{1}{4}}$. The toric curve $S$ on $X$ is defined as the complete intersection

$$
S: \quad P=0, \quad h_{1} \equiv \gamma^{8}\left(x_{1} x_{2} x_{3} x_{4} x_{5}\right)-\alpha^{8} x_{3}^{4}=0, \quad h_{2} \equiv \alpha^{8} x_{1}^{8}-\beta^{8} x_{2}^{8}=0
$$

The Greene-Plesser orbifold group G acts as $x_{i} \rightarrow \lambda_{k}^{g_{k, i}} x_{i}$ with $\lambda_{1}^{8}=\lambda_{2}^{4}=1, \lambda_{3}^{4}=1$ and weights

$$
\mathbb{Z}_{8}: g_{1}=(1,-1,0,0,0), \quad \mathbb{Z}_{4}: g_{2}=(1,0,-1,0,0), \quad \mathbb{Z}_{4}: g_{3}=(1,0,0,-1,0)
$$

Insert $h_{1}$ and $h_{2}$ into $P=0$

$$
\begin{aligned}
\tilde{\mathbb{P}}_{1}: \eta_{1} x_{4}+\sqrt[4]{x_{5}^{4}+m\left(x_{1}, x_{3}\right)} & =0, \quad \eta_{1}^{4}=1 \\
m\left(x_{1}, x_{3}\right) & =\left(\frac{\alpha^{8}+\beta^{8}}{\alpha^{8}}+\phi \frac{\beta^{8}}{\alpha^{8}}\right) x_{1}^{8}+\left(1+\frac{\gamma^{8}}{\alpha^{8}} \psi\right) x_{3}^{4}
\end{aligned}
$$

$\tilde{\mathbb{P}}^{1}$ is a non-holomorphic family due to fourth roots of unity. At critical loci of the parameter space $\alpha, \beta, \gamma$

$$
\mathcal{M}_{\mathbb{P}^{1}}(S): \quad \alpha^{8}+\beta^{8}+\phi \beta^{8}=0, \quad \alpha^{8}+\gamma^{8} \psi=0
$$

$m\left(x_{1}, x_{4}\right)$ vanishes identically and $S$ degenerates to

$$
S: \quad h_{0} \equiv x_{4}^{4}+x_{5}^{4}, \quad h_{1}=\gamma^{8}\left(x_{1} x_{2} x_{3} x_{4} x_{5}\right)-\alpha^{8} x_{3}^{4}=0, \quad h_{2}=\alpha^{8} x_{1}^{8}-\beta^{8} x_{2}^{8}=0
$$

Modulo the action of $G$, (3.23) can be solved holomorphically. Thus the anholomorphic deformation of $\mathbb{P}^{1}$ (3.25) can be used to described holomorphic deformation of $S$.

From the toric data in table 2, we obtain the GKZ-system of $X$ by (2.6),

$$
\begin{aligned}
& \mathcal{Z}_{0}=\sum_{i=0}^{6} \vartheta_{i}+1, \quad \mathcal{Z}_{1}=-\vartheta_{1}+\vartheta_{2}, \quad \mathcal{Z}_{i}=-2 \vartheta_{i}+\vartheta_{i+1}-\vartheta_{6} \quad(i=2,3,4), \\
& \mathcal{L}_{1}=\prod_{i=3}^{6} \frac{\partial}{\partial a_{i}}-\left(\frac{\partial}{\partial a_{0}}\right)^{4}, \quad \mathcal{L}_{2}=\frac{\partial}{\partial a_{1}} \frac{\partial}{\partial a_{2}}-\left(\frac{\partial}{\partial a_{6}}\right)^{2}
\end{aligned}
$$




\begin{tabular}{|c|c|c|c|c|c|c|c|c|c|c|c|c|}
\hline & \multicolumn{7}{|c|}{$\Delta^{\prime}$} & $l_{1}^{\prime}$ & $l_{2}^{\prime}$ & $l_{3}^{\prime}$ & $l_{4}^{\prime}$ & \\
\hline$v_{0}^{\prime}$ & 1 & 0 & 0 & 0 & 0 & 0 & 0 & -3 & 0 & -1 & 0 & $w_{0}^{\prime}=x_{1} x_{2} x_{3} x_{4} x_{5}$ \\
\hline$v_{1}^{\prime}$ & 1 & 0 & -1 & -2 & -2 & -2 & 0 & 0 & 2 & 0 & -1 & $w_{1}^{\prime}=x_{1}^{8}$ \\
\hline$v_{2}^{\prime}$ & 1 & 0 & 1 & 0 & 0 & 0 & 0 & 0 & 0 & 0 & 1 & $w_{2}^{\prime}=x_{2}^{8}$ \\
\hline$v_{3}^{\prime}$ & 1 & 0 & 0 & 1 & 0 & 0 & 0 & 0 & 0 & 1 & 0 & $w_{3}^{\prime}=x_{3}^{4}$ \\
\hline$v_{4}^{\prime}$ & 1 & 0 & 0 & 0 & 1 & 0 & 0 & 1 & 0 & 0 & 0 & $w_{4}^{\prime}=x_{4}^{4}$ \\
\hline$v_{5}^{\prime}$ & 1 & 0 & 0 & 0 & 0 & 1 & 0 & 1 & 0 & 0 & 0 & $w_{5}^{\prime}=x_{5}^{4}$ \\
\hline$v_{6}^{\prime}$ & 1 & 0 & 0 & -1 & -1 & -1 & 0 & 1 & -2 & 0 & 0 & $w_{6}^{\prime}=\left(x_{1} x_{2}\right)^{4}$ \\
\hline$v_{7}^{\prime}$ & 0 & 1 & 0 & 0 & 0 & 0 & -1 & -1 & 0 & 1 & 0 & $w_{7}^{\prime}=y_{1} w_{1}^{\prime}$ \\
\hline$v_{8}^{\prime}$ & 0 & 1 & 0 & 1 & 0 & 0 & -1 & 1 & 0 & -1 & 0 & $w_{8}^{\prime}=y_{1} w_{2}^{\prime}$ \\
\hline$v_{9}^{\prime}$ & 0 & 1 & -1 & -2 & -2 & -2 & 1 & 0 & -1 & 0 & 1 & $w_{9}^{\prime}=y_{2} w_{0}^{e}$ \\
\hline$v_{10}^{\prime}$ & 0 & 1 & 1 & 0 & 0 & 0 & 1 & 0 & 1 & 0 & -1 & $w_{10}^{\prime}=y_{2} w_{3}^{\prime}$ \\
\hline
\end{tabular}

Table 3. Toric Data of $X^{\prime \prime}$ s Mirror Manifold.

where $\mathcal{Z}_{0}$ represents the invariant of $P$ under overall rescaling, $\mathcal{Z}_{i}$ 's relate to the torus symmetry, and $\mathcal{L}_{i}$ 's relate to the symmetries among monomials consisting of $P$. As before, all GKZ operators annihilate the period integrals and determine the mirror maps and superpotentials.

After blowing up $X$ along $S$, we obtain the blow-up manifold defined by,

$$
\begin{aligned}
X^{\prime}: & P=a_{0} x_{1} x_{2} x_{3} x_{4} x_{5}+a_{1} x_{1}^{8}+a_{2} x_{8}+a_{3} x_{4}+a_{4} x_{4}^{4}+a_{5} x_{5}^{4}+a_{6}\left(x_{1} x_{2}\right)^{4}, \\
& Q=y_{1}\left(a_{9} x_{1}^{9}+a_{10} x_{2}^{9}\right)-y_{2}\left(a_{7} x_{1} x_{2} x_{3} x_{4} x_{5}+a_{8} x_{3}^{4}\right)
\end{aligned}
$$

As before, the corresponding infinitesimal generators are obtained, which are belong to the GKZ system of $X^{\prime}$ the GKZ system of $X^{\prime}$ by observation on the torus symmetry,

$$
\begin{aligned}
& \mathcal{Z}_{0}^{\prime}=\sum_{i=0}^{6} \vartheta_{i}+1, \quad \mathcal{Z}_{1}^{\prime}=\sum_{i=7}^{10} \vartheta_{i}, \quad \mathcal{Z}_{2}^{\prime}=-\vartheta_{1}+\vartheta_{2}-\vartheta_{9}+\vartheta_{10}, \quad \mathcal{Z}_{3}^{\prime}=-2 \vartheta_{1}+\vartheta_{3}-\vartheta_{6}+\vartheta_{8}-2 \vartheta_{9}, \\
& \mathcal{Z}_{4}^{\prime}=-2 \vartheta_{1}+\vartheta_{4}-\vartheta_{6}-2 \vartheta_{9}, \quad \mathcal{Z}_{5}^{\prime}=-2 \vartheta_{1}+\vartheta_{5}-\vartheta_{6}-2 \vartheta_{9}, \quad \mathcal{Z}_{6}^{\prime}=-\vartheta_{7}-\vartheta_{8}+\vartheta_{9}+\vartheta_{10} \\
& \mathcal{L}_{1}^{\prime}=\prod_{i=3}^{6} \frac{\partial}{\partial a_{i}}-\left(\frac{\partial}{\partial a_{0}}\right)^{4}, \quad \mathcal{L}_{2}^{\prime}=\prod_{i=1}^{2} \frac{\partial}{\partial a_{i}}-\left(\frac{\partial}{\partial a_{6}}\right)^{2} \\
& \mathcal{L}_{3}^{\prime}=\frac{\partial}{\partial a_{3}} \frac{\partial}{\partial a_{7}}-\frac{\partial}{\partial a_{0}} \frac{\partial}{\partial a_{8}}, \quad \mathcal{L}_{4}^{\prime}=\frac{\partial}{\partial a_{2}} \frac{\partial}{\partial a_{9}}-\frac{\partial}{\partial a_{1}} \frac{\partial}{\partial a_{10}}
\end{aligned}
$$

where $\mathcal{Z}_{0}^{\prime}, \mathcal{Z}_{1}^{\prime}$ are associated with the overall rescaling with respect to $P=0, Q=0$ respectively. $\mathcal{Z}_{i}^{\prime}, i=2, \ldots, 6$ are related to the torus symmetry and The $\mathcal{L}_{3}^{\prime}, \mathcal{L}_{4}^{\prime}$ incorporate the parameter $a_{7}, \ldots, a_{10}$ that are associated with the moduli of the curve $S$.

Then we formulate above GKZ-system in an enhanced polyhedron $\Delta^{\prime}$ (table 3). Here we present the integral points $v_{i}^{\prime}$ of the enhanced polyhedron $\Delta^{\prime}$ and their the corresponding monomials $w_{i}^{\prime}$ s. $l_{i}^{\prime}$ 's are the generators of Mori cone $l_{i}^{\prime}$ satisfying $l^{1}=l_{1}^{\prime}+l_{3}^{\prime}, l^{2}=l_{2}^{\prime}+l_{4}^{\prime}$ and they are the maximal triangulation of $\Delta^{\prime}$. 
By definition (2.7), the local coordinates of complex structure moduli space of $X^{\prime}$ are

$$
z_{1}=\frac{a_{4} a_{5} a_{6} a_{8}}{a_{0}^{3} a_{7}}, \quad z_{2}=\frac{a_{1}^{2} a_{10}}{a_{6}^{2} a_{9}}, \quad z_{3}=\frac{a_{3} a_{7}}{a_{0} a_{8}}, \quad z_{4}=\frac{a_{2} a_{9}}{a_{1} a_{10}}
$$

Next, we convert the $\mathcal{L}_{i}$ operators in (3.28) to Picard-Fuchs operators $\mathcal{D}_{i}$, from differential equations about $a_{j}(j=0, \ldots, 10)$ to those about $z_{j}(j=1, \ldots, 4)$,

$$
\begin{aligned}
& \mathcal{D}_{1}=\theta_{1}^{2}\left(\theta_{1}-2 \theta_{2}\right)\left(\theta_{1}-\theta_{3}\right)-z_{1}\left(-\theta_{1}+\theta_{3}\right) \prod_{i=1}^{3}\left(-3 \theta_{1}-\theta_{3}-i\right), \\
& \mathcal{D}_{2}=\left(2 \theta_{2}-\theta_{4}\right)^{2}\left(\theta_{2}-\theta_{4}\right)-z_{2}\left(\theta_{1}-2 \theta_{2}\right)^{2}\left(-\theta_{2}+\theta_{4}\right) \\
& \mathcal{D}_{3}=\theta_{3}\left(-\theta_{1}+\theta_{3}\right)-z_{3}\left(-3 \theta_{1}-\theta_{3}-1\right)\left(\theta_{1}-\theta_{3}\right), \\
& \mathcal{D}_{4}=\theta_{4}\left(-\theta_{2}+\theta_{4}\right)-z_{4}\left(2 \theta_{2}-\theta_{4}\right)\left(\theta_{2}-\theta_{4}\right)
\end{aligned}
$$

where $\theta_{i}$ 's are the logarithmic derivatives with respect to $z_{i}$ 's and each $D_{i}$ corresponds to a specific linear combination among $l_{1}^{\prime}, l_{2}^{\prime}, l_{3}^{\prime}, l_{4}^{\prime}$.

\subsubsection{Brane superpotential and disk instantons}

Now, we solve the Picard-Fuchs equations (3.30) at $z_{i} \rightarrow 0$ and identified the mirror maps and superpotentials. By the techniques we introduced in section 2.1.

The unique power series solution, as well as the fundamental period of $X$, is

$$
\begin{aligned}
\omega_{0}= & 1+24 z_{1} z_{3}+2520 z_{1}^{2} z_{3}^{2}+369600 z_{1}^{3} z_{3}^{3}+63063000 z_{1}^{4} z_{3}^{4}+5040 z_{1}^{2} z_{2} z_{3}^{2} z_{4} \\
& +2217600 z_{1}^{3} z_{2} z_{3}^{3} z_{4}+\mathcal{O}\left(z^{5}\right)
\end{aligned}
$$

The single logarithmic solutions are

$$
\begin{aligned}
\omega_{1,1}= & \omega_{0} \log \left(z_{1}\right)-6 z_{1}+45 z_{1}^{2}-560 z_{1}^{3}+\frac{17325}{2} z_{1}^{4}-\frac{756756}{5} z_{1}^{5}+z_{3}+78 z_{1} z_{3}-630 z_{1}^{2} z_{3} \\
& +8400 z_{1}^{3} z_{3}-150150 z_{1}^{4} z_{3}-\frac{1}{2} z_{3}^{2}+60 z_{1} z_{3}^{2}+9207 z_{1}^{2} z_{3}^{2}-92400 z_{1}^{3} z_{3}^{2}+\frac{1}{3} z_{3}^{3}-60 z_{1} z_{3}^{3} \\
& +7560 z_{1}^{2} z_{3}^{3}-\frac{1}{4} z_{3}^{4}+70 z_{1} z_{3}^{4}+\frac{1}{5} z_{3}^{5}-z_{2} z_{4}+90 z_{1}^{2} z_{2} z_{4}-3360 z_{1}^{3} z_{2} z_{4}+24 z_{1} z_{2} z_{3} z_{4} \\
& -1260 z_{1}^{2} z_{2} z_{3} z_{4}-\frac{3}{2} z_{2}^{2} z_{4}^{2}+\mathcal{O}\left(z^{5}\right) \\
\omega_{1,2}= & \omega_{0} \log \left(z_{2}\right)+48 z_{1} z_{3}+7560 z_{1}^{2} z_{3}^{2}-2520 z_{1}^{2} z_{2} z_{3}^{2}+2 z_{2} z_{4}-48 z_{1} z_{2} z_{3} z_{4}+3 z_{2}^{2} z_{4}^{2}+\mathcal{O}\left(z^{5}\right) \\
\omega_{1,3}= & \omega_{0} \log \left(z_{3}\right)+6 z_{1}-45 z_{1}^{2}+560 z_{1}^{3}-\frac{17325}{2} z_{1}^{4}+\frac{756756}{5} z_{1}^{5}-z_{3}+26 z_{1} z_{3}+630 z_{1}^{2} z_{3} \\
& -8400 z_{1}^{3} z_{3}+150150 z_{1}^{4} z_{3}+\frac{1}{2} z_{3}^{2}-60 z_{1} z_{3}^{2}+3069 z_{1}^{2} z_{3}^{2}+92400 z_{1}^{3} z_{3}^{2}-\frac{1}{3} z_{3}^{3}+60 z_{1} z_{3}^{3} \\
& -7560 z_{1}^{2} z_{3}^{3}+\frac{1}{4} z_{3}^{4}-70 z_{1} z_{3}^{4}+\frac{1}{5} z_{3}^{5}-90 z_{1}^{2} z_{2} z_{4}+3360 z_{1}^{3} z_{2} z_{4}+1260 z_{1}^{2} z_{2} z_{3} z_{4}+\mathcal{O}\left(z^{5}\right) \\
\omega_{1,4}= & \omega_{0} \log \left(z_{4}\right)+2520 z_{1}^{2} z_{2} z_{3}^{2}+1108800 z_{1}^{3} z_{2} z_{3}^{3}+378378000 z_{1}^{4} z_{2} z_{3}^{4}+252252000 z_{1}^{4} z_{2}^{2} z_{3}^{4} z_{4} \\
& -2520 z_{1}^{2} z_{2} z_{3}^{2} z_{4}^{2}-1108800 z_{1}^{3} z_{2} z_{3}^{3} z_{4}^{2}+\mathcal{O}\left(z^{10}\right)
\end{aligned}
$$


such that single logarithmic period of $X$ can be reproduced by $\Pi_{1}^{1}=\omega_{1,1}+\omega_{1,3}, \Pi_{2}^{1} \omega_{1,2}+\omega_{1,4}$, and open-closed mirror maps are inverse series of flat coordinates,

$$
\begin{aligned}
z_{1}= & q_{1}+6 q_{1}^{2}+9 q_{1}^{3}+56 q_{1}^{4}-300 q_{1}^{5}-q_{1} q_{3}-84 q_{1}^{2} q_{3}-432 q_{1}^{3} q_{3}-900 q_{1}^{4} q_{3}+68 q_{1}^{2} q_{3}^{2} \\
& +4182 q_{1}^{3} q_{3}^{2}+12 q_{1}^{2} q_{3}^{3}+q_{1} q_{2} q_{4}+12 q_{1}^{2} q_{2} q_{4}-63 q_{1}^{3} q_{2} q_{4}-q_{1} q_{2} q_{3} q_{4}-264 q_{1}^{2} q_{2} q_{3} q_{4}+\mathcal{O}\left(q^{5}\right) \\
z_{2}= & q_{2}-48 q_{1} q_{2} q_{3}-264 q_{1}^{2} q_{2} q_{3}^{2}-2 q_{2}^{2} q_{4}+240 q_{1} q_{2}^{2} q_{3} q_{4}+3 q_{2}^{3} q_{4}^{2}+\mathcal{O}\left(q^{5}\right) \\
z_{3}= & q_{3}-6 q_{1} q_{3}+27 q_{1}^{2} q_{3}-164 q_{1}^{3} q_{3}+1377 q_{1}^{4} q_{3}+q_{3}^{2}-32 q_{1} q_{3}^{2}+138 q_{1}^{2} q_{3}^{2}-184 q_{1}^{3} q_{3}^{2}+q_{3}^{3} \\
& -22 q_{1} q_{3}^{3}+675 q_{1}^{2} q_{3}^{3}+q_{3}^{4}-24 q_{1} q_{3}^{4}+q_{3}^{5}-6 q_{1} q_{2} q_{3} q_{4}+144 q_{1}^{2} q_{2} q_{3} q_{4}-32 q_{1} q_{2} q_{3}^{2} q_{4}+\mathcal{O}\left(q^{5}\right) \\
z_{4}= & q_{4}-2520 q_{1}^{2} q_{2} q_{3}^{2} q_{4}-403200 q_{1}^{3} q_{2} q_{3}^{3} q_{4}-53701200 q_{1}^{4} q_{2} q_{3}^{4} q_{4}-403200 q_{1}^{3} q_{2}^{2} q_{3}^{3} q_{4}^{2} \\
& +2520 q_{1}^{2} q_{2} q_{3}^{2} q_{4}^{3}+403200 q_{1}^{3} q_{2} q_{3}^{3} q_{4}^{3}+\mathcal{O}\left(q^{10}\right)
\end{aligned}
$$

The double logarithmic solutions are denoted by their leading term

$$
\begin{array}{llll}
\frac{1}{2} \ell_{1}^{2}, & \frac{1}{2} \ell_{2}^{2}, & \frac{1}{2} \ell_{3}^{2}+\ell_{1} \ell_{3}, & \frac{1}{2} \ell_{4}^{2}+\ell_{2} \ell_{4}, \\
\ell_{1} \ell_{2}, & \ell_{1} \ell_{4}, & \ell_{2} \ell_{3}, & \ell_{3} \ell_{4}
\end{array}
$$

with abbreviations $\ell_{i}=\log \left(z_{i}\right)$. The brane superpotential is constructed as linear combination of double logarithmic solutions,

$$
\mathcal{W}_{\text {brane }}=t_{1}^{2}+\sum_{n_{i}} N_{d_{1}, d_{2}, d_{3}, d_{4}} \operatorname{Li}_{2}\left(q_{1}^{d_{1}} q_{2}^{d_{2}} q_{3}^{d_{3}} q_{3}^{d_{4}}\right)
$$

This has the expected integrality properties of the Ooguri-Vafa $\mathrm{Li}_{2}$ multicover formula. The Ooguri-Vafa invariants of the form $N_{(\mathrm{m}, \mathrm{k}, \mathrm{m}+\mathrm{n}, \mathrm{k})}$ are exactly match the data in table 5 in [24]. In addition, we also extract the invariants of the form $N_{(\mathrm{m}+\mathrm{n}, \mathrm{k}, \mathrm{n}, \mathrm{k})}$ and summarize them into table 11, where the rows and columns are labelled by $\mathrm{m}$ and $\mathrm{n}$, respectively.

3.3 Open-closed GKZ-system: branes on $X_{12}^{(1,1,2,2,6)}$

\subsubsection{Five branes wrapping rational curves and blowing up geometry}

In this section, we study the A-model manifold, whose toric polyhedron is denoted by $\Delta^{*}$ and charge vectors are denoted by $l^{1}$ and $l^{2}$. A-model manifold and A-brane are specified by the following toric data.

The mirror hypersurface $X$ is determined by the constraint,

$$
P=x_{1}^{12}+x_{2}^{12}+x_{3}^{6}+x_{4}^{6}+x_{5}^{2}+\psi\left(x_{1} x_{2} x_{3} x_{4} x_{5}\right)+\phi\left(x_{1} x_{2}\right)^{6}
$$

where $x_{i}$ 's are homogeneous coordinates in $\mathbb{P}_{(1,1,2,2,6)}$ and $\psi=z_{1}^{-\frac{1}{6}} z_{2}^{-\frac{1}{12}}, \phi=z_{2}^{-\frac{1}{2}}$. The Greene-Plesser orbifold group G acts as $x_{i} \rightarrow \lambda_{k}^{g, i} x_{i}$ with $\lambda_{1}^{6}=\lambda_{2}^{6}=\lambda_{3}^{2}=1$ and weights

$$
\mathbb{Z}_{6}: g_{1}=(1,0,-1,0,0), \quad \mathbb{Z}_{6}: g_{2}=(1,0,0,-1,0), \quad \mathbb{Z}_{2}: g_{3}=(1,0,0,0,-1)
$$

The toric curve $S$ is described by the complete intersection

$$
S: \quad P=0, \quad h_{1} \equiv \gamma^{12}\left(x_{1} x_{2} x_{3} x_{4} x_{5}\right)-\alpha^{12} x_{5}^{2}=0, \quad h_{2} \equiv \alpha^{12} x_{1}^{12}-\beta^{12} x_{2}^{12}=0
$$




\begin{tabular}{|c|c|c|c|c|c|c|c|c|c|}
\hline & \multicolumn{4}{|c|}{$\Delta^{*}$} & \multicolumn{2}{|c|}{$l^{1} \quad l^{2}$} & \multirow[b]{2}{*}{$x_{1} x_{2} x_{3} x_{4} x_{5}$} & \multirow{2}{*}{$\begin{array}{l}\hat{l}^{1} \\
-1\end{array}$} & $\hat{l}^{2}$ \\
\hline$v_{0}^{*}$ & 0 & 0 & 0 & 0 & -6 & 0 & & & 0 \\
\hline$v_{1}^{*}$ & -1 & -2 & -2 & -6 & 0 & 1 & $x_{1}^{12}$ & 0 & -1 \\
\hline$v_{2}^{*}$ & 1 & 0 & 0 & 0 & 0 & 1 & $x_{2}^{12}$ & 0 & 1 \\
\hline$v_{3}^{*}$ & 0 & 1 & 0 & 0 & 1 & 0 & $x_{3}^{6}$ & 0 & 0 \\
\hline$v_{4}^{*}$ & 0 & 0 & 1 & 0 & 1 & 0 & $x_{4}^{6}$ & 0 & 0 \\
\hline$v_{5}^{*}$ & 0 & 0 & 0 & 1 & 3 & 0 & $x_{5}^{2}$ & 1 & 0 \\
\hline$v_{6}^{*}$ & 0 & -1 & -1 & -3 & 1 & -2 & $\left(x_{1} x_{2}\right)^{6}$ & 0 & 0 \\
\hline
\end{tabular}

Table 4. Toric Data of A-model side.

Insert $h_{1}$ and $h_{2}$ into $P=0$

$$
\begin{aligned}
\tilde{\mathbb{P}}^{1}: \quad \eta_{1} x_{3}+\sqrt[6]{x_{4}^{6}+m\left(x_{1}, x_{5}\right)} & =0, \quad \eta_{1}^{6}=1 \\
m\left(x_{1}, x_{5}\right) & =\left(\frac{\alpha^{12}+\beta^{12}}{\alpha^{12}}+\frac{\beta^{12}}{\alpha^{12}} \phi\right) x_{1}^{12}+\left(1+\frac{\gamma^{12}}{\alpha^{12}} \phi\right) x_{5}^{2}
\end{aligned}
$$

Here $\tilde{\mathbb{P}} 1_{\text {is }}$ evidently non-holomorphic because of the sixth roots of unity and hence, a nonholomorphic family of rational curves on. However, at special loci

$$
\alpha^{12}+\beta^{12}+\phi \beta^{12}=0, \quad \alpha^{12}+\gamma^{12} \psi=0
$$

we see that $S$ degenerates as follows,

$$
\Sigma: \quad h_{0} \equiv x_{3}^{6}+x_{4}^{6}, \quad h_{1} \equiv \gamma^{12}\left(x_{1} x_{2} x_{3} x_{4} x_{5}\right)-\alpha^{12} x_{5}^{2}=0, \quad h_{2} \equiv \alpha^{12} x_{1}^{12}-\beta^{12} x_{2}^{12}=0
$$

Modulo the action of G, (3.32) can be solve holomorphically. Thus the anholomorphic deformation of $\mathbb{P}^{1}$ (3.34) can be used to describe holomorphic deformation of $S$.

From the toric data in table 4, the full set of GKZ operators are derived by (2.6)

$$
\begin{aligned}
& \mathcal{Z}_{0}=\sum_{i=0}^{6} \vartheta_{i}+1 \\
& \mathcal{Z}_{1}=\vartheta_{1}-\vartheta_{2} \\
& \mathcal{Z}_{2}=2 \vartheta_{1}-\vartheta_{3}+\vartheta_{6}, \\
& \mathcal{Z}_{3}=2 \vartheta_{1}-\vartheta_{4}+\vartheta_{6}, \quad \mathcal{Z}_{4}=6 \vartheta_{1}-\vartheta_{5}+3 \vartheta_{6}, \\
& \mathcal{L}_{1}=\frac{\partial}{\partial a_{1}} \frac{\partial}{\partial a_{2}}-\left(\frac{\partial}{\partial a_{6}}\right)^{6}, \quad \mathcal{L}_{2}=\frac{\partial}{\partial a_{3}} \frac{\partial}{\partial a_{4}}\left(\frac{\partial}{\partial a_{5}}\right)^{3} \frac{\partial}{\partial a_{6}}-\left(\frac{\partial}{\partial a_{0}}\right)^{6}
\end{aligned}
$$

where $\mathcal{Z}_{0}$ represents the invariance of $P$ under overall rescaling, $\mathcal{Z}_{i}$ 's relate to the torus symmetry, and $\mathcal{L}_{i}$ 's relate to the symmetries among monomials consisting of $P$. And all GKZ operators above annihilate the period matrix and determine the mirror maps and superpotential.

After blowing up $X$ along $S$, the blow-up manifold $X^{\prime}$ is obtained as the complete intersection in $\mathcal{W}=\mathbb{P}(\mathcal{O}(12) \oplus \mathbb{P O}(12))$

$$
\begin{aligned}
X^{\prime}: & P=a_{1} x_{1}^{12}+1_{2} x_{2}^{12}+a_{3} x_{3}^{6}+a_{4} x_{4}^{6}+a_{5} x_{5}^{2}+a_{0}\left(x_{1} x_{2} x_{3} x_{4} x_{5}\right)+a_{6}\left(x_{1} x_{2}\right)^{6}=0 \\
& Q=y_{1}\left(a_{9} x_{1}^{12}+a_{10} x_{2}^{12}\right)-y_{2}\left(a_{7}\left(x_{1} x_{2} x_{3} x_{4} x_{5}\right)+a_{8} x_{3}^{6}\right)
\end{aligned}
$$


where $a_{i}$ 's are free complex-valued coefficients. By simple observation, we can obtain the GKZ system of $X^{\prime}$ as complement to GKZ system of $X$.

$$
\begin{aligned}
& \mathcal{Z}_{0}^{\prime}=\sum_{i=0}^{6} \vartheta_{i}+1, \quad \mathcal{Z}_{1}^{\prime}=\sum_{i=7}^{10} \vartheta_{i}, \quad \mathcal{Z}_{2}^{\prime}=-\vartheta_{1}+\vartheta_{2}-\vartheta_{9}+\vartheta_{10}, \quad \mathcal{Z}_{3}^{\prime}=-2 \vartheta_{1}+\vartheta_{3}-\vartheta_{6}-2 \vartheta_{9}, \\
& \mathcal{Z}_{4}^{\prime}=-2 \vartheta_{1}+\vartheta_{4}-\vartheta_{6}-2 \vartheta_{9}, \quad \mathcal{Z}_{5}^{\prime}=-6 \vartheta_{1}+\vartheta_{5}-3 \vartheta_{6}+\vartheta_{8}-6 \vartheta_{9}, \quad \mathcal{Z}_{6}^{\prime}=-\vartheta_{7}-\vartheta_{8}+\vartheta_{9}+\vartheta_{10} \\
& \mathcal{L}_{1}^{\prime}=\prod_{i=1}^{3} \frac{\partial}{\partial a_{i}}-\left(\frac{\partial}{\partial a_{0}}\right)^{3}, \quad \mathcal{L}_{2}^{\prime}=\prod_{i=4}^{6} \frac{\partial}{\partial a_{i}}-\left(\frac{\partial}{\partial a_{3}}\right)^{3} \\
& \mathcal{L}_{3}^{\prime}=\frac{\partial}{\partial a_{3}} \frac{\partial}{\partial a_{8}}-\frac{\partial}{\partial a_{5}} \frac{\partial}{\partial a_{7}}, \quad \mathcal{L}_{4}^{\prime}=\frac{\partial}{\partial a_{6}} \frac{\partial}{\partial a_{9}}-\frac{\partial}{\partial a_{3}} \frac{\partial}{\partial a_{10}}
\end{aligned}
$$

where $\mathcal{Z}_{0}^{\prime}, \mathcal{Z}_{1}^{\prime}$ are associated with the overall rescaling with respect to $P=0, Q=0$,

\begin{tabular}{|c|c|c|c|c|c|c|c|c|c|c|c|c|}
\hline & \multicolumn{7}{|c|}{$\Delta^{\prime}$} & $l_{1}^{\prime}$ & $l_{2}^{\prime}$ & $l_{3}^{\prime}$ & $l_{4}^{\prime}$ & \\
\hline$v_{0}^{\prime}$ & 1 & 0 & 0 & 0 & 0 & 0 & 0 & -1 & -3 & 0 & 0 & $w_{0}^{\prime}=x_{1} x_{2} x_{3} x_{4} x_{5}$ \\
\hline$v_{1}^{\prime}$ & 1 & 0 & -1 & -2 & -2 & -6 & 0 & 0 & 0 & 2 & -1 & $w_{1}^{\prime}=x_{1}^{12}$ \\
\hline$v_{2}^{\prime}$ & 1 & 0 & 1 & 0 & 0 & 0 & 0 & 0 & 0 & 0 & 1 & $w_{2}^{\prime}=x_{2}^{12}$ \\
\hline$v_{3}^{\prime}$ & 1 & 0 & 0 & 1 & 0 & 0 & 0 & 0 & 1 & 0 & 0 & $w_{3}^{\prime}=x_{3}^{6}$ \\
\hline$v_{4}^{\prime}$ & 1 & 0 & 0 & 0 & 1 & 0 & 0 & 0 & 1 & 0 & 0 & $w_{4}^{\prime}=x_{4}^{6}$ \\
\hline$v_{5}^{\prime}$ & 1 & 0 & 0 & 0 & 0 & 1 & 0 & 1 & 0 & 0 & 0 & $w_{5}^{\prime}=x_{5}^{2}$ \\
\hline$v_{6}^{\prime}$ & 1 & 0 & 0 & -1 & -1 & -3 & 0 & 0 & 1 & -2 & 0 & $w_{6}^{\prime}=\left(x_{1} x_{2}\right)^{6}$ \\
\hline$v_{7}^{\prime}$ & 0 & 1 & 0 & 0 & 0 & 0 & -1 & 1 & -3 & 0 & 0 & $w_{7}^{\prime}=y_{1} w_{1}^{\prime}$ \\
\hline$v_{8}^{\prime}$ & 0 & 1 & 0 & 0 & 0 & 1 & -1 & -1 & 3 & 0 & 0 & $w_{8}^{\prime}=y_{1} w_{2}^{\prime}$ \\
\hline$v_{9}^{\prime}$ & 0 & 1 & -1 & -2 & -2 & -6 & 1 & 0 & 0 & -1 & 1 & $w_{9}^{\prime}=y_{2} w_{0}^{\prime}$ \\
\hline$v_{10}^{\prime}$ & 0 & 1 & 1 & 0 & 0 & 0 & 1 & 0 & 0 & 1 & -1 & $w_{10}^{\prime}=y_{2} w_{5}^{e}$ \\
\hline
\end{tabular}
$\mathcal{Z}_{i}^{\prime}, i=2, \ldots, 6$ are related to the torus symmetry, and $\mathcal{L}_{3}^{\prime}, \mathcal{L}_{4}^{\prime}$ incorporate the parameter $a_{7}, \ldots, a_{10}$ that are associated with the moduli of the curve $S$.

Then we formulate above GKZ-system on an enhanced polyhedron $\Delta^{\prime}$

Here we present the integral points $v_{i}^{\prime}$ s and their corresponding monomials $w_{i}^{\prime}$, as well as $l_{i}^{\prime}$ 's as basis of Mori cone satisfying relations $l^{1}=3 l_{1}^{\prime}+l_{2}^{\prime}$ and $l^{2}=l_{3}^{\prime}+l_{4}^{\prime}$.

The local coordinates $z^{i}$ 's on the complex structure moduli space of $X^{\prime}$ by $(2.7)$,

$$
z_{1}=-\frac{a_{5} a_{7}}{a_{0}^{3} a_{8}}, \quad z_{2}=\frac{a_{3} a_{4} a_{6} a_{8}^{3}}{a_{0}^{3} a_{7}^{3}}, \quad z_{3}=\frac{a_{1}^{2} a_{10}}{a_{6}^{2} a_{9}}, \quad z_{4}=\frac{a_{2} a_{9}}{a_{1} a_{10}}
$$

Next, we convert the $\mathcal{L}_{i}$ in (3.37)operators to Picard-Fuchs operators $\mathcal{D}_{i}$, from differential equations about $a_{j}(j=0, \ldots, 10)$ to those about $z_{j}(j=1, \ldots, 4)$,

$$
\begin{aligned}
& \mathcal{D}_{1}=\theta_{1}\left(\theta_{1}-3 \theta_{2}-1\right)-z_{1}\left(-\theta_{1}-3 \theta_{2}\right)\left(-\theta_{1}+3 \theta_{2}\right) \\
& \mathcal{D}_{2}=\theta_{2}^{2}\left(\theta_{2}-2 \theta_{3}\right)\left(-\theta_{1}+3 \theta_{2}\right)^{3}-z_{2}\left(\theta_{1}-3 \theta_{2}\right)^{3} \prod_{i=1}^{3}\left(-\theta_{1}-3 \theta_{2}-i\right)
\end{aligned}
$$




$$
\begin{aligned}
& \mathcal{D}_{3}=\left(2 \theta_{3}-\theta_{4}\right)^{2}\left(\theta_{3}-\theta_{4}\right)-z_{3}\left(\theta_{2}-2 \theta_{3}\right)^{2}\left(-\theta_{3}+\theta_{4}\right), \\
& \mathcal{D}_{4}=\theta_{4}\left(-\theta_{3}+\theta_{4}\right)-z_{4}\left(2 \theta_{3}-\theta_{4}\right)\left(\theta_{3}-\theta_{4}\right)
\end{aligned}
$$

where $\theta_{i}=z_{i} \frac{\partial}{\partial z_{i}}$ 's are the logarithmic derivatives and each $\mathcal{D}_{i}$ corresponds to a specific linear combination among $l_{i}^{\prime}$ s.

\subsubsection{Brane superpotential and disk instantons}

Along the line of 2.1, we solve the differential equations (3.38) at $z_{i} \rightarrow 0$ and identify the mirror maps and superpotential. The fundamental period of $X$ as power series solution is

$$
\omega_{0}=1-120 z_{1}^{3} z_{2}+83160 z_{1}^{6} z_{2}^{2}+166320 z_{1}^{6} z_{2}^{2} z_{3} z_{4}+\mathcal{O}\left(z^{10}\right) .
$$

The single logarithmic solutions are

$$
\begin{aligned}
\omega_{1,1}= & \omega_{0} \log \left(z_{1}\right)+z_{1}+\frac{1}{2} z_{1}^{2}+\frac{1}{3} z_{1}^{3}+\frac{1}{4} z_{1}^{4}+\frac{1}{5} z_{1}^{5}+2 z_{2}+12 z_{1} z_{2}+60 z_{1}^{2} z_{2}-74 z_{1}^{3} z_{2}-210 z_{1}^{4} z_{2} \\
& -15 z_{2}^{2}-126 z_{1} z_{2}^{2}-630 z_{1}^{2} z_{2}^{2}-2520 z_{1}^{3} z_{2}^{2}+\frac{560}{3} z_{2}^{3}+2100 z_{1} z_{2}^{3}+13200 z_{1}^{2} z_{2}^{3}-\frac{5775}{2} z_{2}^{4} \\
& -40950 z_{1} z_{2}^{4}+\frac{252252}{5} z_{2}^{5}-30 z_{2}^{2} z_{3} z_{4}-252 z_{1} z_{2}^{2} z_{3} z_{4}+1120 z_{2}^{3} z_{3} z_{4}+\mathcal{O}\left(z^{6}\right) \\
\omega_{1,2}= & \omega_{0} \log \left(z_{2}\right)-3 z_{1}-\frac{3}{2} z_{1}^{2}-z_{1}^{3}-\frac{3}{4} z_{1}^{4}-\frac{3}{5} z_{1}^{5}-6 z_{2}-36 z_{1} z_{2}-180 z_{1}^{2} z_{2}-522 z_{1}^{3} z_{2}+630 z_{1}^{4} z_{2} \\
& +45 z_{2}^{2}+378 z_{1} z_{2}^{2}+1890 z_{1}^{2} z_{2}^{2}+7560 z_{1}^{3} z_{2}^{2}-560 z_{2}^{3}-6300 z_{1} z_{2}^{3}-39600 z_{1}^{2} z_{2}^{3}+\frac{17325}{2} z_{2}^{4} \\
& +122850 z_{1} z_{2}^{4}-\frac{756756}{5} z_{2}^{5}-z_{3} z_{4}+90 z_{2}^{2} z_{3} z_{4}+756 z_{1} z_{2}^{2} z_{3} z_{4}-3360 z_{2}^{3} z_{3} z_{4}-\frac{3}{2} z_{3}^{2} z_{4}^{2}+\mathcal{O}\left(z^{6}\right) \\
\omega_{1,3}= & \omega_{0} \log \left(z_{3}\right)-240 z 1^{3} z 2+2 z 3 z 4+240 z 1^{3} z 2 z 3 z 4+3 z 3^{2} z 4^{2}+120 z 1^{3} z 2 z 3^{2} z 4^{2}+\frac{20}{3} z 3^{3} z 4^{3} \\
& +160 z 1^{3} z 2 z 3^{3} z 4^{3}+\frac{35}{2} z 3^{4} z 4^{4}+\frac{252}{5} z 3^{5} z 4^{5}++\mathcal{O}\left(z^{10}\right) \\
\omega_{1,4}= & \omega_{0} \log \left(z_{4}\right)
\end{aligned}
$$

such that the single logarithmic periods of $X$ are reproduced by $\Pi_{1}^{1}=\omega_{1,1}+\omega_{1,2}, \Pi_{2}^{1}=$ $\omega_{1,3}+\omega_{1,4}$. With single logarithmic solutions, open-closed mirror maps are inverse series of flat coordinates

$$
\begin{aligned}
z_{1}= & q_{1}-q_{1}^{2}+q_{1}^{3}-q_{1}^{4}+q_{1}^{5}-2 q_{1} q_{2}-14 q_{1}^{2} q_{2}-60 q_{1}^{3} q_{2}+134 q_{1}^{4} q_{2}+5 q_{1} q_{2}^{2}+58 q_{1}^{2} q_{2}^{2} \\
& +270 q_{1}^{3} q_{2}^{2}-32 q_{1} q_{2}^{3}-546 q_{1}^{2} q_{2}^{3}+286 q_{1} q_{2}^{4}-2 q_{1} q_{2} q_{3} q_{4}-14 q_{1}^{2} q_{2} q_{3} q_{4}+40 q_{1} q_{2}^{2} q_{3} q_{4}+\mathcal{O}\left(q^{5}\right) \\
z_{2}= & q_{2}+3 q_{1} q_{2}+3 q_{1}^{2} q_{2}+q_{1}^{3} q_{2}+6 q_{2}^{2}+66 q_{1} q_{2}^{2}+384 q_{1}^{2} q_{2}^{2}+1338 q_{1}^{3} q_{2}^{2}+9 q_{2}^{3}+222 q_{1} q_{2}^{3} \\
& +2940 q_{1}^{2} q_{2}^{3}+56 q_{2}^{4}+1350 q_{1} q_{2}^{4}-300 q_{2}^{5}+q_{2} q_{3} q_{4}+3 q_{1} q_{2} q_{3} q_{4}+3 q_{1}^{2} q_{2} q_{3} q_{4} \\
& +12 q_{2}^{2} q_{3} q_{4}+132 q_{1} q_{2}^{2} q_{3} q_{4}-63 q_{2}^{3} q_{3} q_{4}+\mathcal{O}\left(q^{5}\right) \\
z_{3}= & q_{3}+240 q_{1}^{3} q_{2} q_{3}-2 q_{3}^{2} q_{4}-1200 q_{1}^{3} q_{2} q_{3}^{2} q_{4}+3 q_{3}^{3} q_{4}^{2}+3120 q_{1}^{3} q 2 q_{3}^{3} q_{4}^{2}-4 q_{3}^{4} q_{4}^{3}+5 q_{3}^{5} q_{4}^{4}+\mathcal{O}\left(q^{10}\right) \\
z_{4}= & q_{4}
\end{aligned}
$$


The double logarithmic solutions are denoted by their leading term

$$
\begin{array}{llll}
\frac{3}{2} \ell_{1}^{2}+\ell_{1} \ell_{2}, & \frac{1}{2} \ell_{2}^{2}, & \frac{1}{2} \ell_{3}^{2}, & \frac{1}{2} \ell_{4}^{2}+\ell_{3} \ell_{4}, \\
\ell_{1} \ell_{3}, & \ell_{1} \ell_{4}, & \ell_{2} \ell_{3}, & \ell_{2} \ell_{4}
\end{array}
$$

with abbreviations $\ell_{i}=\log \left(z_{i}\right)$. Then we construct two linear combination of double logarithmic solutions and insert the inverse mirror maps to match the disk instantons in $[10]$.

$$
\begin{aligned}
& \mathcal{W}_{\text {brane }}^{I}=\frac{3}{2} t_{1}^{2}+t_{1} t_{2}+\sum_{N^{I}} N_{d_{1}, d_{2}, d_{3}, d_{4}}^{I} \operatorname{Li}_{2}\left(q_{1}^{d_{1}} q_{2}^{d_{2}} q_{3}^{d_{3}} q_{4}^{d_{4}}\right) \\
& \mathcal{W}_{\text {brane }}^{I I}=\frac{1}{2} t_{1} t_{3}+\sum_{N^{I I}} N_{d_{1}, d_{2}, d_{3}, d_{4}}^{I I} \operatorname{Li}_{2}\left(q_{1}^{d_{1}} q_{2}^{d_{2}} q_{3}^{d_{3}} q_{4}^{d_{4}}\right) .
\end{aligned}
$$

Invariants of the form $N_{\mathrm{m}, \mathrm{n}, \mathrm{k}, \mathrm{k}}$ are summarized in table 12,13,14,15, where the rows and columns are labelled by $\mathrm{m}$ and $\mathrm{n}$, respectively.

\section{One closed and two open moduli case}

\subsection{Open-closed GKZ-system: branes on complete intersections $\mathbb{P}_{[3,3]}^{(111 \mid 111)}$}

\subsubsection{Five branes wrapping lines and blowing up geometry}

The underlying manifold $X^{*}$ we are considering in the A-model is the intersection of two cubics in $\mathbb{P}^{5}$, whose mirror manifold $X$ can be represented a one-parameter family of bicubics, with group $G=\mathbb{Z}_{3}^{2} \times \mathbb{Z}_{9}$ acting on them,

$$
\left\{\begin{array}{l}
P_{1}=x_{1}^{3}+x_{2}^{3}+x_{3}^{3}+\psi x_{4} x_{5} x_{6} \\
P_{2}=x_{4}^{3}+x_{5}^{3}+x_{6}^{3}+\psi x_{1} x_{2} x_{3}
\end{array}\right.
$$

where $\psi$ is the complex structure modulus.

Turning to the specification of D-brane configurations, we consider the curve $S$ on $X$

$$
\begin{aligned}
& P_{1}=P_{2}=0, \\
& h_{1} \equiv \beta^{3}\left(x_{1} x_{2} x_{3}\right)-\alpha \beta \gamma x_{2}^{3}=0, \\
& h_{2} \equiv \gamma^{3}\left(x_{1} x_{2} x_{3}\right)-\alpha \beta \gamma x_{3}^{3}=0
\end{aligned}
$$

An equivalent and convenient form is easy to obtained,

$$
P_{1}=P_{2}=0, \quad \alpha^{3} x_{2}^{3}-\beta^{3} x_{1}^{3}=0, \quad \alpha^{3} x_{3}^{3}-\gamma^{3} x_{1}^{3}=0
$$

For generic values of the moduli, the $S$ is an irreducible higher genus Riemann surface. But we can always make a linearization by inserting $h_{1}$ and $h_{2}$ into $P_{1}, P_{2}$,

$$
x_{4}^{3}+x_{5}^{3}+x_{6}^{3}-\Psi x_{4} x_{5} x_{6}=0, \quad \Psi=\frac{\alpha \beta \gamma}{\alpha^{3}+\beta^{3}+\gamma^{3}} \psi^{2}
$$




\begin{tabular}{|c|c|c|c|c|c|c|c|c|c|}
\hline & \multicolumn{5}{|c|}{$\Delta^{*}$} & $l$ & & $\hat{l}^{1}$ & $\hat{l}^{2}$ \\
\hline$v_{0}^{*}$ & 0 & 0 & 0 & 0 & 0 & -3 & $x_{1} x_{2} x_{3}$ & -1 & -1 \\
\hline$v_{0}^{*}$ & 0 & 0 & 0 & 0 & 0 & -3 & $x_{4} x_{5} x_{6}$ & 0 & 0 \\
\hline$v_{1}^{*}$ & -1 & -1 & -1 & -1 & -1 & 1 & $x_{1}^{3}$ & 0 & 0 \\
\hline$v_{2}^{*}$ & 1 & 0 & 0 & 0 & 0 & 1 & $x_{2}^{3}$ & 1 & 0 \\
\hline$v_{3}^{*}$ & 0 & 1 & 0 & 0 & 0 & 1 & $x_{3}^{3}$ & 0 & 1 \\
\hline$v_{4}^{*}$ & 0 & 0 & 1 & 0 & 0 & 1 & $x_{4}^{3}$ & 0 & 0 \\
\hline$v_{5}^{*}$ & 0 & 0 & 0 & 1 & 0 & 1 & $x_{5}^{3}$ & 0 & 0 \\
\hline$v_{6}^{*}$ & 0 & 0 & 0 & 0 & 1 & 1 & $x_{6}^{3}$ & 0 & 0 \\
\hline
\end{tabular}

Table 5. Toric Data of A-model side.

This is an one dimensional family of cubic plane elliptic curves in $\mathbb{P}^{2}$, called the Hesse pencil. For special value of $\Psi$, it degenerate into 12 lines [25],

$$
x_{4}+\eta_{1} x_{5}+\eta_{2} x_{6}=0, \quad \eta_{1}^{3}=\eta_{2}^{3}=1
$$

Upon the action of group G, they are identified as a single line.

Thus the deformation space of (4.4) is embedded in the deformation space of $S$. And away from that special locus, the obstructed deformation is identified with the unobstructed deformation of $S$, which means that we can use the obstructed deformation of that line to describe the unobstructed deformation of $S$.

The polyhedron corresponding to the A-model manifold $X^{*}$ is denoted as $\Delta^{*}$, the Abrane charge vectors $l$, and A-brane charge vectors $\hat{l}^{1}, \hat{l}^{2}$. The toric data in A-model side is as table 5 .

In toric coordinates, the Calabi-Yau threefold $X$ and the curve $S$ are described as

$$
\begin{aligned}
X: & P_{1}=a_{0,1}+a_{1}\left(X_{2} X_{3} X_{4} X_{5} X_{6}\right)^{-1}+a_{2} X_{2}+a_{3} X_{3}=0 \\
& P_{2}=a_{0,2}+a_{4} X_{4}+a_{5} X_{5}+a_{6} X_{6}=0 \\
S: & h_{1}=a_{7}+a_{8} X_{2}=0, \quad h_{2}=a_{9}+a_{10} X_{3}=0
\end{aligned}
$$

with $a_{i}$ 's are free complex-valued coefficients. The GKZ-system as follow by (2.6),

$$
\begin{aligned}
& \mathcal{Z}_{0}=\sum_{i=0}^{6} \vartheta_{i}+1, \quad \mathcal{Z}_{i}=-\vartheta_{1}+\vartheta_{i+1}, i=1, \ldots, 5 \\
& \mathcal{L}_{1}=\prod_{i=1}^{6} \frac{\partial}{\partial a_{i}}-\left(\frac{\partial}{\partial a_{0,1}}\right)^{3}\left(\frac{\partial}{\partial a_{0,2}}\right)^{3}
\end{aligned}
$$

where $\vartheta_{i}=a_{i} \frac{\partial}{\partial a_{i}}$ 's are the logarithmic derivative. $\mathcal{Z}_{0}$ represents the invariance of $P$ under overall rescaling and $\mathcal{Z}_{i}$ 's relate to the torus symmetry,

$$
\mathcal{Z}_{i}: \quad X_{i+1} \mapsto \lambda X_{i+1}, \quad\left(a_{1}, a_{i+1}\right) \mapsto\left(\lambda a_{1}, \lambda^{-1} a_{i+1}\right), \quad i=1,2,3,4
$$


For $\mathcal{L}_{i}$ 's, they represents the relations among Laurent monomials in $P_{1}$ and $P_{2}$ in (4.1).

$$
\mathcal{L}_{1}: \quad\left(X_{2}^{-1}\right)\left(X_{3}^{-1}\right)\left(X_{4}^{-1}\right)\left(X_{5}^{-1}\right)\left(X_{6}^{-1}\right) X_{2} X_{3} X_{4} X_{5} X_{6}=1
$$

And all GKZ operators annihilate the period matrix and determine the mirror maps and superpotentials.

After blowing up $X$ along $S$, the blow-up manifold is

$$
X^{\prime}: P_{1}=P_{2}=0, \quad Q=y_{1}\left(a_{9}+a_{10} X_{3}\right)-y_{2}\left(a_{7}+a_{8} X_{2}\right)
$$

By careful observations on the defining equations (4.6), the GKZ system of $X^{\prime}$ is obtained as follow

$$
\begin{aligned}
\mathcal{Z}_{0}^{\prime} & =\sum_{i=0}^{6} \vartheta_{i}+1, \quad \mathcal{Z}_{1}^{\prime}=\sum_{i=7}^{10} \vartheta_{i}, \quad \mathcal{Z}_{2}^{\prime}=-\vartheta_{1}+\vartheta_{2}+\vartheta_{8}, \quad \mathcal{Z}_{3}^{\prime}=-\vartheta_{1}+\vartheta_{3}+\vartheta_{10}, \\
\mathcal{Z}_{i}^{\prime} & =-\vartheta_{1}+\vartheta_{i}, \quad i=4,5,6, \quad \mathcal{Z}_{7}^{\prime}=-\vartheta_{7}-\vartheta_{8}+\vartheta_{9}+\vartheta_{10} \\
\mathcal{L}_{1}^{\prime} & =\prod_{i=1}^{3} \frac{\partial}{\partial a_{i}}-\left(\frac{\partial}{\partial a_{0}}\right)^{3}, \\
\mathcal{L}_{2}^{\prime} & =\frac{\partial}{\partial a_{2}} \frac{\partial}{\partial a_{7}}-\frac{\partial}{\partial a_{0,1}} \frac{\partial}{\partial a_{8}}, \quad \mathcal{L}_{3}^{\prime}=\frac{\partial}{\partial a_{3}} \frac{\partial}{\partial a_{9}}-\frac{\partial}{\partial a_{0,1}} \frac{\partial}{\partial a_{10}}
\end{aligned}
$$

where $\mathcal{Z}_{0}^{\prime}, \mathcal{Z}_{1}^{\prime}$ are associated with the overall rescaling with respect to $P=0, Q=0$

\begin{tabular}{|c|c|c|c|c|c|c|c|c|c|c|c|c|}
\hline & \multicolumn{8}{|c|}{$\Delta^{\prime}$} & & $l_{2}^{\prime}$ & $l_{3}^{\prime}$ & \\
\hline$v_{0}^{\prime}$ & 1 & 0 & 0 & 0 & 0 & 0 & 0 & 0 & 0 & -1 & -1 & $w_{0,1}^{\prime}=x_{1} x_{2} x_{3}$ \\
\hline$v_{0}^{\prime}$ & 1 & 0 & 0 & 0 & 0 & 0 & 0 & 0 & -3 & 0 & 0 & $w_{0,2}^{\prime}=x_{4} x_{5} x_{6}$ \\
\hline$v_{1}^{\prime}$ & 1 & 0 & -1 & -1 & -1 & -1 & -1 & 0 & 1 & 0 & 0 & $w_{1}^{\prime}=x_{1}^{3}$ \\
\hline$v_{2}^{\prime}$ & 1 & 0 & 1 & 0 & 0 & 0 & 0 & 0 & -1 & 1 & 0 & $w_{2}^{\prime}=x_{2}^{3}$ \\
\hline$v_{3}^{\prime}$ & 1 & 0 & 0 & 1 & 0 & 0 & 0 & 0 & 0 & 0 & 1 & $w_{3}^{\prime}=x_{3}^{3}$ \\
\hline$v_{4}^{\prime}$ & 1 & 0 & 0 & 0 & 1 & 0 & 0 & 0 & 1 & 0 & 0 & $w_{4}^{\prime}=x_{4}^{3}$ \\
\hline$v_{5}^{\prime}$ & 1 & 0 & 0 & 0 & 0 & 1 & 0 & 0 & 1 & 0 & 0 & $w_{5}^{\prime}=x_{5}^{3}$ \\
\hline$v_{6}^{\prime}$ & 1 & 0 & 0 & 0 & 0 & 0 & 1 & 0 & 1 & 0 & 0 & $w_{6}^{\prime}=x_{6}^{3}$ \\
\hline$v_{7}^{\prime}$ & 0 & 1 & 0 & 0 & 0 & 0 & 0 & -1 & -2 & 1 & 0 & $w_{7}^{\prime}=y_{1} w_{0}^{\prime}$ \\
\hline$v_{8}^{\prime}$ & 0 & 1 & 1 & 0 & 0 & 0 & 0 & -1 & 2 & -1 & 0 & $w_{8}^{\prime}=y_{1} w_{2}^{\prime}$ \\
\hline$v_{9}^{\prime}$ & 0 & 1 & 0 & 0 & 0 & 0 & 0 & 1 & -1 & 0 & 1 & $w_{9}^{\prime}=y_{2} w_{0}^{\prime}$ \\
\hline$v_{10}^{\prime}$ & 0 & 1 & 0 & 1 & 0 & 0 & 0 & 1 & 1 & 0 & -1 & $w_{10}^{\prime}=y_{2} w_{3}^{\prime}$ \\
\hline
\end{tabular}
respectively. $\mathcal{Z}_{i}^{\prime}, i=2, \ldots, 5$ are related to the torus symmetry.

$$
\begin{aligned}
\mathcal{Z}_{2}^{\prime}: & X_{2} & \mapsto \lambda X_{2}, & \left(a_{1}, a_{2}, a_{8}\right) & \mapsto\left(\lambda a_{1}, \lambda^{-1} a_{2}, \lambda^{-1} a_{8}\right) & \\
\mathcal{Z}_{3}^{\prime}: & X_{3} & \mapsto \lambda X_{3}, & \left(a_{1}, a_{3}, a_{10}\right) & \mapsto\left(\lambda a_{1}, \lambda^{-1} a_{3}, \lambda^{-1} a_{10}\right) & \\
\mathcal{Z}_{i}^{\prime}: & X_{i} & \mapsto \lambda X_{i}, & \left(a_{1}, a_{i}\right) & \mapsto\left(\lambda a_{1}, \lambda^{-1} a_{i}\right), & i=4,5,6
\end{aligned}
$$

In addition, $\mathcal{Z}_{6}^{\prime}$ is related to the torus symmetry $\left(y_{1}, y_{2}\right) \mapsto\left(\lambda y_{1}, \lambda^{-1} y_{2}\right)$. The $\mathcal{L}_{2}^{\prime}, \mathcal{L}_{3}^{\prime}$ incorporate the parameter $a_{7}, \ldots, a_{10}$ that are associated with the moduli of the curve $S$.

Now, we formulate GKZ system (4.7) on an enhanced polyhedron $\Delta^{\prime}$, 
where $v_{i}^{\prime}$ s are the integral vertices and $w_{i}^{\prime}$, their corresponding monomials. The A-model closed string charge vectors and A-branes charge vectors satisfy the relations $l^{1}=l_{1}^{\prime}+2 l_{2}^{\prime}+$ $l_{3}^{\prime}, \hat{l}^{1}=l_{3}^{\prime}, \hat{l}^{2}=l_{4}^{\prime}$.

The coordinates $z_{i}$ by (2.7)on the complex structure moduli space of $X^{\prime}$,

$$
z_{1}=\frac{a_{1} a_{4} a_{5} a_{6} a_{8} a_{10}}{a_{0,2}^{3} a_{2} a_{7}^{2} a_{9}}, \quad z_{2}=\frac{a_{2} a_{7}}{a_{0,1} a_{8}}, \quad z_{3}=\frac{a_{3} a_{9}}{a_{0,1} a_{10}}
$$

Next, we convert the $\mathcal{L}_{i}$ operators in (4.7) to Picard-Fuchs operators $\mathcal{D}_{i}$, from differential equations about $a_{j}$ to those about $z_{j}$,

$$
\begin{aligned}
& \mathcal{D}_{1}=\theta_{1}^{4}\left(2 \theta_{1}-\theta_{2}\right)^{2}\left(\theta_{1}-\theta_{3}\right)-z_{1}\left(-\theta_{1}+\theta_{2}\right)\left(-2 \theta_{1}+\theta_{2}\right)^{2}\left(-\theta_{1}+\theta_{3}\right) \prod_{i=1}^{3}\left(-3 \theta_{1}-i\right), \\
& \mathcal{D}_{2}=\left(-\theta_{1}+\theta_{2}\right)\left(-2 \theta_{1}+\theta_{2}\right)-z_{2}\left(-\theta_{2}-\theta_{3}-1\right)\left(2 \theta_{1}-\theta_{2}\right) \\
& \mathcal{D}_{3}=\theta_{3}\left(-\theta_{1}+\theta_{3}\right)-z_{3}\left(-\theta_{2}-\theta_{3}-1\right)\left(\theta_{1}-\theta_{3}\right),
\end{aligned}
$$

where $\theta_{i}=z_{i} \frac{\partial}{\partial z_{i}}$ 's are the logarithmic derivatives and each operator $\mathcal{D}_{a}$ corresponds to a linear combination among the charge vectors $l_{1}^{\prime}, l_{2}^{\prime}, l_{3}^{\prime}$.

\subsubsection{Brane superpotential and disk instantons}

Now, we solve the Picard-Fuchs equations (4.9)derived in the last section and identified the mirror maps and superpotentials. By the methods introduced in 2.1, at $z_{i} \rightarrow 0$, the fundamental period of $X$ as series expansion is

$$
\omega_{0}=1+36 z_{1} z_{2}^{2} z_{3}+8100 z_{1}^{2} z_{2}^{4} z_{3}^{2}+2822400 z_{1}^{3} z_{2}^{6} z_{3}^{3}+1200622500 z_{1}^{4} z_{2}^{8} z_{3}^{4}+\mathcal{O}\left(z^{16}\right)
$$

There are four the single logarithmic solutions

$$
\begin{aligned}
\omega_{1,1}= & \omega_{0} \log \left(z_{1}\right)+2 z_{2}-z_{2}^{2}-12 z_{1} z_{2}^{2}+\frac{2}{3} z_{2}^{3}-\frac{1}{2} z_{2}^{4}+\frac{2}{5} z_{2}^{5}+z_{3}-24 z_{1} z_{2} z_{3}+90 z_{1} z_{2}^{2} z_{3} \\
& +144 z_{1} z_{2}^{3} z_{3}-\frac{1}{2} z_{3}^{2}+72 z_{1} z_{2}^{2} z_{3}^{2}+\frac{1}{3} z_{3}^{3}-\frac{1}{4} z_{3}^{4}+\frac{1}{5} z_{3}^{5}+\mathcal{O}\left(z^{5}\right) \\
\omega_{1,2}= & \omega_{0} \log \left(z_{2}\right)-z_{2}+\frac{1}{2} z_{2}^{2}-\frac{1}{3} z_{2}^{3}+\frac{1}{4} z_{2}^{4}-\frac{1}{5} z_{2}^{5}+12 z_{1} z_{2} z_{3}+30 z_{1} z_{2}^{2} z_{3}-72 z_{1} z_{2}^{3} z_{3}+\mathcal{O}\left(z^{6}\right) \\
\omega_{1,3}= & \omega_{0} \log \left(z_{3}\right)+12 z_{1} z_{2}^{2}-270 z_{1}^{2} z_{2}^{4}-z_{3}+30 z_{1} z_{2}^{2} z_{3}+\frac{1}{2} z_{3}^{2}-72 z_{1} z_{2}^{2} z_{3}^{2}-\frac{1}{3} z_{3}^{3}+\frac{1}{4} z_{3}^{4} \\
& -\frac{1}{5} z_{3}^{5}+\mathcal{O}\left(z^{5}\right)
\end{aligned}
$$

such that they are consistent with the single logarithmic periods of $X, \Pi^{1}=\omega_{1,1}+2 \omega_{1,2}+\omega_{1,3}$.

By the definition of the flat coordinates and mirror maps

$$
\begin{aligned}
t_{j} & =\frac{\omega_{1, j}}{\omega_{0}} \\
q_{j} & =e^{2 \pi i t_{j}}
\end{aligned}
$$


we obtain the $z_{j}$ as a series of $q_{j}$ upon inversion of the mirror maps

$$
\begin{aligned}
z_{1}= & q_{1}-2 q_{1} q_{2}+q_{1} q_{2}^{2}+12 q_{1}^{2} q_{2}^{2}-24 q_{1}^{2} q_{2}^{3}-q_{1} q_{3}+2 q_{1} q_{2} q_{3}+24 q_{1}^{2} q_{2} q_{3}-q_{1} q_{2}^{2} q_{3} \\
& \quad-150 q_{1}^{2} q_{2}^{2} q_{3}-24 q_{1}^{2} q_{2} q_{3}^{2}+\mathcal{O}\left(q^{5}\right) \\
z_{2}= & q_{2}+q_{2}^{2}+q_{2}^{3}+q_{2}^{4}+q_{2}^{5}-12 q_{1} q_{2}^{2} q_{3}-42 q_{1} q_{2}^{3} q_{3}+\mathcal{O}\left(q^{5}\right) \\
z_{3}= & q_{3}-12 q_{1} q_{2}^{2} q_{3}+q_{3}^{2}-42 q_{1} q_{2}^{2} q_{3}^{2}+q_{3}^{3}+q_{3}^{4}+q_{3}^{5}+\mathcal{O}\left(q^{5}\right)
\end{aligned}
$$

In addition, there are also double logarithmic solutions with leading terms

$$
\frac{1}{2} \ell_{1}^{2}-\ell_{2}^{2}, \quad \frac{3}{2} \ell_{2}^{2}+\ell_{1} \ell_{2}, \quad \frac{1}{2} \ell_{3}^{2}+\ell_{1} \ell_{3}, \quad \ell_{2} \ell_{3}
$$

where $\log \left(z_{i}\right)$ 's are abbreviated as $\ell_{i}$ 's. According to above, a specific linear combination of double logarithmic solutions is constructed and its disk instantons expansions are extracted.

$$
\mathcal{W}_{\text {brane }}=4 t_{1} t_{2}+6 t_{2}^{2}+4 t_{2} t_{3}+\sum_{N} N_{d_{1}, d_{2}, d_{3}} \operatorname{Li}_{2}\left(q_{1}^{d_{1}} q_{2}^{d_{2}} q_{3}^{d_{3}}\right)
$$

We present first a few invariants of the form $N_{m, m+n, m}$ in table 16 .

\subsection{Branes on complete intersections $\mathbb{P}_{[4,4]}^{(112 \mid 112)}$ and $\mathbb{P}_{[6,6]}^{(123 \mid 123)}$}

Similar to the last section, we also calculate the superpotential and extract the OoguriVafa invariants at large volume phase for complete intersections Calabi-Yau manifolds of $\mathbb{P}_{[4,4]}^{(112 \mid 112)}$ and $\mathbb{P}_{[6,6]}^{(123 \mid 123)}$. We summarize the main formulas and tables in appendix $A$ and Ooguri-Vafa invariants for first several orders in appendix C.

\section{One closed and three open moduli case}

\subsection{Open-closed GKZ-system: branes on sextic hypersurface}

\subsubsection{Branes wrapping rational curves and blowing up geometry}

In this section, we consider the A-model manifold $X^{*}$ with charge vector $l=(-6,1,1,1,1,2)$. The corresponding toric polyhedron for $X^{*}$ consists of following integral vertices

$$
(0,0,0,0), \quad(-1,-1,-1,-2), \quad(1,0,0,0), \quad(0,1,0,0), \quad(0,0,1,0), \quad(0,0,0,1)
$$

The mirror sextic hypersurface $X$ arises as the Calabi-Yau hypersurfacesis a mirror pair of Calabi-Yau hypersurface in $\mathbb{P}_{(1,1,1,1,2)}^{4}$. Its defining equation in homogeneous coordinates is

$$
P=a_{1} x_{1}^{6}+a_{2} x_{2}^{6}+a_{3} x_{3}^{6}+a_{4} x_{4}^{6}+a_{5} x_{5}^{3}+a_{0} x_{1} x_{2} x_{3} x_{4} x_{5} .
$$

On $X$, we consider parallel branes $S$ which are described by

$S: \quad P=0, \quad h_{1} \equiv a_{6}\left(x_{1} x_{2} x_{3} x_{4} x_{5}\right)^{2}+a_{7} x_{1} x_{2} x_{3} x_{4} x_{5}^{4}+a_{8} x_{5}^{6}=0, \quad h_{2} \equiv a_{9} x_{1}^{6}+a_{10} x_{5}^{3}=0$

The GKZ operators are derived by (2.6)

$$
\begin{aligned}
\mathcal{Z}_{0} & =\sum_{i=0}^{5} \vartheta_{i}+1, \quad \mathcal{Z}_{i}=\vartheta_{i+1}-\vartheta_{1}, i=1,2,3, \quad \mathcal{Z}_{4}=\vartheta_{5}-2 \vartheta_{1} \\
\mathcal{L}_{1} & =\frac{\partial}{\partial a_{1}} \frac{\partial}{\partial a_{2}} \frac{\partial}{\partial a_{3}} \frac{\partial}{\partial a_{4}}{\frac{\partial}{\partial a_{5}}}^{2}-\left(\frac{\partial}{\partial a_{0}}\right)^{6}
\end{aligned}
$$


where $\mathcal{Z}_{0}$ represents the invariance of $P$ under overall rescaling, $\mathcal{Z}_{i}$ 's relate to the torus symmetry, and $\mathcal{L}_{i}$ 's relate to the symmetries among monomials consisting of $P$. And all GKZ operators above annihilate the period matrix and determine the mirror maps and superpotential.

After blowing up $X$ along $S$, the blow-up manifold is obtained as the complete intersection in the projective bundle

$$
\begin{aligned}
X^{\prime}: & P=a_{1} x_{1}^{6}+x_{2}^{6}+x_{3}^{6}+x_{4}^{6}+x_{5}^{3}+a_{0}\left(x_{1} x_{2} x_{3} x_{4} x_{5}\right)=0 \\
Q & =y_{1}\left(a_{9} x_{1}^{6}+a_{10} x_{5}^{3}\right)-y_{2}\left(a_{6}\left(x_{1} x_{2} x_{3} x_{4} x_{5}\right)^{2}+a_{7} x_{1} x_{2} x_{3} x_{4} x_{5}^{4}+a_{8} x_{5}^{6}\right)
\end{aligned}
$$

where $a_{i}$ 's are free complex-valued coefficients. By observation on the symmetry of obove defining equations, we can obtain the GKZ system of $X^{\prime}$ as complement to GKZ system of $X$.

$$
\begin{aligned}
& \mathcal{Z}_{0}^{\prime}=\sum_{i=0}^{5} \vartheta_{i}+1, \quad \mathcal{Z}_{1}^{\prime}=\sum_{i=6}^{10} \vartheta_{i}, \quad \mathcal{Z}_{2}^{\prime}=-\vartheta_{1}+\vartheta_{2}+\vartheta_{7}+2 \vartheta_{8}-\vartheta_{9}, \quad \mathcal{Z}_{3}^{\prime}=-1 \vartheta_{1}+\vartheta_{3}-\vartheta_{9}, \\
& \mathcal{Z}_{4}^{\prime}=-\vartheta_{1}+\vartheta_{4}-\vartheta_{9}, \quad \mathcal{Z}_{5}^{\prime}=-2 \vartheta_{1}+\vartheta_{5}-2 \vartheta_{6}+\vartheta_{8}-6 \vartheta_{9}, \quad \mathcal{Z}_{6}^{\prime}=-\vartheta_{6}-\vartheta_{7}-\vartheta_{8}+\vartheta_{9}+\vartheta_{10} \\
& \mathcal{L}_{1}^{\prime}=\prod_{i=2}^{5} \frac{\partial}{\partial a_{i}}\left(\frac{\partial}{\partial a_{7}}\right)^{2} \frac{\partial}{\partial a_{9}}-\left(\frac{\partial}{\partial a_{0}}\right)^{4} \frac{\partial}{\partial a_{6}} \frac{\partial}{\partial a_{10}}, \quad \mathcal{L}_{2}^{\prime}=\frac{\partial}{\partial a_{6}} \frac{\partial}{\partial a_{8}}-\left(\frac{\partial}{\partial a_{7}}\right)^{2} \\
& \mathcal{L}_{3}^{\prime}=\frac{\partial}{\partial a_{5}} \frac{\partial}{\partial a_{7}}-\frac{\partial}{\partial a_{0}} \frac{\partial}{\partial a_{8}}, \quad \mathcal{L}_{4}^{\prime}=\frac{\partial}{\partial a_{1}} \frac{\partial}{\partial a_{10}}-\frac{\partial}{\partial a_{5}} \frac{\partial}{\partial a_{9}}
\end{aligned}
$$

where $\mathcal{Z}_{0}^{\prime}, \mathcal{Z}_{1}^{\prime}$ are associated with the overall rescaling with respect to $P=0, Q=0$, $\mathcal{Z}_{i}^{\prime}, i=2, \ldots, 6$ are related to the torus symmetry, and $\mathcal{L}_{3}^{\prime}, \mathcal{L}_{4}^{\prime}$ incorporate the parameter $a_{6}, \ldots, a_{10}$ that are associated with the moduli of the curve $S$. The new manifold $X^{\prime}$ is describe by the following charge vectors

\begin{tabular}{c|ccccccccccc}
\hline & 0 & 1 & 2 & 3 & 4 & 5 & 6 & 7 & 8 & 9 & 10 \\
\hline$l_{1}^{\prime}$ & -4 & 0 & 1 & 1 & 1 & 1 & -2 & 2 & 0 & 1 & -1 \\
$l_{2}^{\prime}$ & 0 & 0 & 0 & 0 & 0 & 0 & 1 & -2 & 1 & 0 & 0 \\
$l_{3}^{\prime}$ & -1 & 0 & 0 & 0 & 0 & 1 & 0 & 1 & -1 & 0 & 0 \\
$l_{4}^{\prime}$ & 0 & -1 & 1 & 0 & 0 & 0 & 0 & 0 & 0 & 1 & -1 \\
\hline
\end{tabular}

The local coordinates $z^{i}$ 's on the complex structure moduli space of $X^{\prime}$ by (2.7) is,

$$
z_{1}=\frac{a_{2} a_{3} a_{4} a_{5} a_{7}^{2} a_{9}}{a_{0}^{4} a_{6}^{2} a_{10}}, \quad z_{2}=\frac{a_{6} a_{8}}{a_{7}^{2}}, \quad z_{3}=\frac{a_{5} a_{7}}{a_{0} a_{8}}, \quad z_{4}=\frac{a_{1} a_{10}}{a_{5} a_{9}}
$$

Next, we convert the $\mathcal{L}_{i}^{\prime}$ in (5.4)operators to Picard-Fuchs operators $\mathcal{D}_{i}$, from differential equations about $a_{j}(j=0, \ldots, 10)$ to those about $z_{j}(j=1, \ldots, 4)$,

$$
\begin{aligned}
& \mathcal{D}_{1}=\theta_{1}^{3}\left(\theta_{1}+\theta_{3}-\theta_{4}\right)\left(2 \theta_{1}-2 \theta_{2}+\theta_{3}\right)^{2}\left(\theta_{1}-\theta_{4}\right)-z_{1}\left(-2 \theta_{1}+\theta_{2}\right)^{2}\left(-\theta_{1}+\theta_{4}\right) \prod_{i=1}^{4}\left(-4 \theta_{1}-\theta_{3}-i\right), \\
& \mathcal{D}_{2}=\left(-2 \theta_{1}+\theta_{2}\right)\left(\theta_{2}-\theta_{3}\right)-z_{2}\left(2 \theta_{2}-2 \theta_{2}+\theta_{3}\right)^{2}
\end{aligned}
$$




$$
\begin{aligned}
& \mathcal{D}_{3}=\left(\theta_{1}+\theta_{3}-\theta_{4}\right)\left(2 \theta_{1}-2 \theta_{2}+\theta_{3}\right)-z_{3}\left(\theta_{2}-\theta_{3}\right)\left(-4 \theta_{1}-\theta_{3}-1\right), \\
& \mathcal{D}_{4}=\theta_{4}\left(-\theta_{1}+\theta_{4}\right)-z_{4}\left(\theta_{1}+\theta_{3}-\theta_{4}\right)\left(\theta_{1}-\theta_{4}\right)
\end{aligned}
$$

where $\theta_{i}=z_{i} \frac{\partial}{\partial z_{i}}$ 's are the logarithmic derivatives.

\subsubsection{Brane superpotential and disk instantons}

Along the line of 2.1, we solve the differential equations (5.6) at $z_{i} \rightarrow 0$ and identify the mirror maps and superpotential. The fundamental period of $X$ is

$$
\omega_{0}=1+360 z_{1} z_{2}^{2} z_{3}^{2} z_{4}+1247400 z_{1}^{2} z_{2}^{4} z_{3}^{4} z_{4}^{2}+6861254400 z_{1}^{3} z_{2}^{6} z_{3}^{6} z_{4}^{3}+\mathcal{O}\left(z^{10}\right) .
$$

The single logarithmic solutions are

$$
\begin{aligned}
\omega_{1,1}= & \omega_{0} \log \left(z_{1}\right)-2 z_{2}-3 z_{2}^{2}-\frac{20}{3} z_{2}^{3}-\frac{35}{2} z_{2}^{4}-\frac{252}{5} z_{2}^{5}+2 z_{2} z_{3}+2 z_{2}^{2} z_{3}+4 z_{2}^{3} z_{3}+10 z_{2}^{4} z_{3}-z_{2}^{2} z_{3}^{2} \\
& -120 z_{1} z_{2}^{2} z_{3}^{2}-2 z_{2}^{3} z_{3}^{2}+24 z_{1} z_{4}-48 z_{1} z_{2} z_{4}-24 z_{1} z_{2}^{2} z_{4}-48 z_{1} z_{2}^{3} z_{4}-240 z_{1} z_{2} z_{3} z_{4} \\
& +240 z_{1} z_{2}^{2} z_{3} z_{4}+1260 z_{1}^{2} z_{4}^{2}-5040 z_{1}^{2} z_{2} z_{4}^{2}++\mathcal{O}\left(z^{5}\right) \\
\omega_{1,2}= & \omega_{0} \log \left(z_{2}\right)+2 z_{2}+3 z_{2}^{2}+\frac{20}{3} z_{2}^{3}+\frac{35}{2} z_{2}^{4}+\frac{252}{5} z_{2}^{5}+z_{3}-2 z_{2} z_{3}-2 z_{2}^{2} z_{3}-4 z_{2}^{3} z_{3}-10 z_{2}^{4} z_{3} \\
& -\frac{1}{2} z_{3}^{2}+z_{2} z_{3}^{2}+z_{2}^{2} z_{3}^{2}+2 z_{2}^{3} z_{3}^{2}+\frac{1}{3} z_{3}^{3}-z_{2} z_{3}^{3}-\frac{1}{4} z_{3}^{4}+z_{2} z_{3}^{4}-12 z_{1} z_{4}+24 z_{1} z_{2} z_{4}+24 z_{1} z_{2}^{2} z_{4} \\
& +48 z_{1} z_{2}^{3} z_{4}+120 z_{1} z_{2} z_{3} z_{4}-240 z_{1} z_{2}^{2} z_{3} z_{4}-630 z_{1}^{2} z_{4}^{2}+2520 z_{1}^{2} z_{2} z_{4}^{2}+\mathcal{O}\left(z^{5}\right) \\
\omega_{1,3}= & \omega_{0} \log \left(z_{3}\right)-z_{2}-\frac{3}{2} z_{2}^{2}-\frac{10}{3} z_{2}^{3}-\frac{35}{4} z_{2}^{4}-\frac{126}{5} z_{2}^{5}-z_{3}+z_{2} z_{3}+z_{2}^{2} z_{3}+2 z_{2}^{3} z_{3}+5 z_{2}^{4} z_{3}+ \\
& \frac{1}{2} z_{3}^{2}-z_{2} z_{3}^{2}-\frac{1}{2} z_{2}^{2} z_{3}^{2}-z_{2}^{3} z_{3}^{2}-\frac{1}{3} z_{3}^{3}+z_{2} z_{3}^{3}+\frac{1}{4} z_{3}^{4}-z_{2} z_{3}^{4}-\frac{1}{5} z_{3}^{5}-12 z_{1} z_{2}^{2} z_{4}-24 z_{1} z_{2}^{3} z_{4}+\mathcal{O}\left(z^{5}\right) \\
\omega_{1,4}= & \omega_{0} \log \left(z_{4}\right)+120 z_{1} z_{2}^{2} z_{3}^{2}-41580 z_{1}^{2} z_{2}^{4} z_{3}^{4}+180 z_{1} z_{2}^{2} z_{3}^{2} z_{4}+498960 z_{1}^{2} z_{2}^{4} z_{3}^{4} z_{4} \\
& -360 z_{1} z_{2}^{2} z_{3}^{2} z_{4}^{2}+727650 z_{1}^{2} z_{2}^{4} z_{3}^{4} z_{4}^{2}+60 z_{1} z_{2}^{2} z_{3}^{2} z_{4}^{3}+\mathcal{O}\left(z^{10}\right)
\end{aligned}
$$

by which the open-closed mirror maps are inverse series of flat coordinates

$$
\begin{aligned}
& z_{1}=q_{1}+2 q_{1} q_{2}+q_{1} q_{2}^{2}-4 q_{1} q_{2} q_{3}-4 q_{1} q_{2}^{2} q_{3}+4 q_{1} q_{2}^{2} q_{3}^{2}+\mathcal{O}\left(q^{5}\right) \\
& z_{2}=q_{2}-2 q_{2}^{2}+3 q_{2}^{3}-4 q_{2}^{4}+5 q_{2}^{5}-q_{2} q_{3}+5 q_{2}^{2} q_{3}-13 q_{2}^{3} q_{3}+25 q_{2}^{4} q_{3}-3 q_{2}^{2} q_{3}^{2}+18 q_{2}^{3} q_{3}^{2}+\mathcal{O}\left(q^{5}\right) \\
& z_{3}=q_{3}+q_{2} q_{3}+q_{3}^{2}+q_{2}^{2} q_{3}^{2}+q_{3}^{3}+q_{3}^{4}+q_{3}^{5}+\mathcal{O}\left(q^{5}\right) \\
& z_{4}=q_{4}-120 q_{1} q_{2}^{2} q_{3}^{2} q_{4}-180 q_{1} q_{2}^{2} q_{3}^{2} q_{4}^{2}+\mathcal{O}\left(q^{7}\right)
\end{aligned}
$$

Then we construct a linear combination of double logarithmic solutions and insert the inverse mirror maps.

$$
\mathcal{W}_{\text {brane }}=3 t_{1}^{2}+6 t_{1} t_{4}+3 t_{4}^{2}+\sum_{N} N_{d_{1}, d_{2}, d_{3}, d_{4}} \operatorname{Li}_{2}\left(q_{1}^{d_{1}} q_{2}^{d_{2}} q_{3}^{d_{3}} q_{4}^{d_{4}}\right)
$$

Invariants of the form $N_{\mathrm{m}, \mathrm{m}, \mathrm{m}, \mathrm{n}}$ are summarized in table 17, where the rows and columns are labelled by $m$ and $n$, respectively. 
When $a_{7}^{2}=a_{6} a_{8}$, the two individual branes coincide. We obtain a new set of charge vectors,

\begin{tabular}{c|cccccccccc}
\hline & 0 & 1 & 2 & 3 & 4 & 5 & 6 & 8 & 9 & 10 \\
\hline$l_{1}^{c}$ & -4 & 0 & 1 & 1 & 1 & 1 & -1 & 1 & 1 & -1 \\
$l_{2}^{c}$ & -2 & 0 & 0 & 0 & 0 & 2 & 1 & -1 & 0 & 0 \\
$l_{3}^{c}$ & 0 & -1 & 1 & 0 & 0 & 0 & 0 & 0 & 1 & -1 \\
\hline
\end{tabular}

by which the new complex structure moduli space coordinates are

$$
z_{1}^{c}=\frac{a_{2} a_{3} a_{4} a_{5} a_{8} a_{9}}{a_{0}^{4} a_{6} a_{10}}, \quad z_{2}^{c}=\frac{a_{5}^{2} a_{6}}{a_{0}^{2} a_{8}}, \quad z_{3}^{c}=\frac{a_{2} a_{9}}{a_{1} a_{10}}
$$

Similar to the separate case, the superpotential is constructed as linear combination of double logarithmic solutions and Ooguri-Vafa invariants are extracted in table 17.

\subsection{Open-closed GKZ-system: branes on sextic hypersurface}

\subsubsection{Branes wrapping rational curves and blowing up geometry}

The mirror octic hypersurface $X$ arises as the Calabi-Yau hypersurfaces in $\mathbb{P}_{(1,1,1,1,4)}^{4}$

$$
P=a_{1} x_{1}^{8}+a_{2} x_{2}^{8}+a_{3} x_{3}^{8}+a_{4} x_{4}^{8}+a_{5} x_{5}^{2}+a_{0} x_{1} x_{2} x_{3} x_{4} x_{5}
$$

on which we consider parallel branes which are described by intersections of divisors

$S: \quad P=0, \quad h_{1} \equiv a_{6}\left(x_{1} x_{2} x_{3} x_{4} x_{5}\right)^{2}+a_{7} x_{1} x_{2} x_{3} x_{4} x_{5}^{3}+a_{8} x_{5}^{4}=0, \quad h_{2} \equiv a_{9} x_{1}^{8}+a_{10} x_{5}^{2}=0$

The GKZ operators are derived by (2.6)

$$
\begin{aligned}
\mathcal{Z}_{0} & =\sum_{i=0}^{5} \vartheta_{i}+1, \quad \mathcal{Z}_{i}=\vartheta_{i+1}-\vartheta_{1}, \quad i=1,2,3, \quad \mathcal{Z}_{4}=\vartheta_{5}-4 \vartheta_{1} \\
\mathcal{L}_{1} & =\frac{\partial}{\partial a_{1}} \frac{\partial}{\partial a_{2}} \frac{\partial}{\partial a_{3}} \frac{\partial}{\partial a_{4}}{\frac{\partial}{\partial a_{5}}}^{4}-\left(\frac{\partial}{\partial a_{0}}\right)^{8}
\end{aligned}
$$

where $\mathcal{Z}_{0}$ represents the invariance of $P$ under overall rescaling, $\mathcal{Z}_{i}$ 's relate to the torus symmetry, and $\mathcal{L}_{i}$ 's relate to the symmetries among monomials consisting of $P$. And all GKZ operators above annihilate the period matrix and determine the mirror maps and superpotential.

After blowing up $X$ along $S$, the blow-up manifold $X^{\prime}$ is obtained as the complete intersection

$$
\begin{aligned}
X^{\prime}: \quad P & =a_{1} x_{1}^{8}+x_{2}^{8}+x_{3}^{8}+x_{4}^{8}+x_{5}^{2}+a_{0}\left(x_{1} x_{2} x_{3} x_{4} x_{5}\right)=0 \\
Q & =y_{1}\left(a_{9} x_{1}^{8}+a_{10} x_{5}^{2}\right)-y_{2}\left(a_{6}\left(x_{1} x_{2} x_{3} x_{4} x_{5}\right)^{2}+a_{7} x_{1} x_{2} x_{3} x_{4} x_{5}^{3}+a_{8} x_{5}^{4}\right)
\end{aligned}
$$


where $a_{i}$ 's are free complex-valued coefficients. By observation on above defining equations, GKZ system of $X^{\prime}$ is obtained as complement to GKZ system of $X$.

$$
\begin{aligned}
\mathcal{Z}_{0}^{\prime} & =\sum_{i=0}^{5} \vartheta_{i}+1, \quad \mathcal{Z}_{1}^{\prime}=\sum_{i=6}^{10} \vartheta_{i}, \quad \mathcal{Z}_{2}^{\prime}=-\vartheta_{1}+\vartheta_{2}+\vartheta_{7}+2 \vartheta_{8}-\vartheta_{1} 0, \quad \mathcal{Z}_{3}^{\prime}=-\vartheta_{1}+\vartheta_{3}-\vartheta_{10}, \\
\mathcal{Z}_{4}^{\prime} & =-\vartheta_{1}+\vartheta_{4}-\vartheta_{10}, \quad \mathcal{Z}_{5}^{\prime}=-4 \vartheta_{1}+\vartheta_{5}-4 \vartheta_{10}, \quad \mathcal{Z}_{6}^{\prime}=-\vartheta_{6}-\vartheta_{7}-\vartheta_{8}+\vartheta_{9}+\vartheta_{10} \\
\mathcal{L}_{1}^{\prime} & =\prod_{i=2}^{4} \frac{\partial}{\partial a_{i}}\left(\frac{\partial}{\partial a_{7}}\right)^{4} \frac{\partial}{\partial a_{10}}-\left(\frac{\partial}{\partial a_{0}}\right)^{3}\left(\frac{\partial}{\partial a_{6}}\right)^{4} \frac{\partial}{\partial a_{9}}, \quad \mathcal{L}_{2}^{\prime}=\frac{\partial}{\partial a_{6}} \frac{\partial}{\partial a_{8}}-\left(\frac{\partial}{\partial a_{7}}\right)^{2} \\
\mathcal{L}_{3}^{\prime} & =\frac{\partial}{\partial a_{5}} \frac{\partial}{\partial a_{7}}-\frac{\partial}{\partial a_{0}} \frac{\partial}{\partial a_{8}}, \quad \mathcal{L}_{4}^{\prime}=\frac{\partial}{\partial a_{1}} \frac{\partial}{\partial a_{9}}-\frac{\partial}{\partial a_{0}} \frac{\partial}{\partial a_{10}}
\end{aligned}
$$

where $\mathcal{Z}_{0}^{\prime}, \mathcal{Z}_{1}^{\prime}$ are associated with the overall rescaling with respect to $P=0, Q=0$, $\mathcal{Z}_{i}^{\prime}, i=2, \ldots, 5$ are related to the torus symmetry, and $\mathcal{L}_{3}^{\prime}, \mathcal{L}_{4}^{\prime}$ incorporate the parameter $a_{6}, \ldots, a_{10}$ that are associated with the moduli of the curve $S$. The new manifold $X^{\prime}$ is describe by the following charge vectors

\begin{tabular}{c|ccccccccccc}
\hline & 0 & 1 & 2 & 3 & 4 & 5 & 6 & 7 & 8 & 9 & 10 \\
\hline$l_{1}^{\prime}$ & -3 & 0 & 1 & 1 & 1 & 0 & -4 & 4 & 0 & -1 & 1 \\
$l_{2}^{\prime}$ & 0 & 0 & 0 & 0 & 0 & 0 & 1 & -2 & 1 & 0 & 0 \\
$l_{3}^{\prime}$ & -1 & 0 & 0 & 0 & 0 & 1 & 0 & 1 & -1 & 0 & 0 \\
$l_{4}^{\prime}$ & -1 & 1 & 0 & 0 & 0 & 0 & 0 & 0 & 0 & -1 & 1 \\
\hline
\end{tabular}

The local coordinates $z^{i}$ 's on the complex structure moduli space of $X^{\prime}$ by $(2.7)$,

$$
z_{1}=\frac{a_{3} a_{4} a_{5} a_{7}^{4} a_{10}}{a_{0}^{4} a_{9}}, \quad z_{2}=\frac{a_{6} a_{8}}{a_{7}^{2}}, \quad z_{3}=\frac{a_{5} a_{7}}{a_{0} a_{8}}, \quad z_{4}=\frac{a_{1} a_{9}}{a_{0} a_{10}}
$$

Next, we convert the $\mathcal{L}_{i}^{\prime}$ in (3.37)operators to Picard-Fuchs operators $\mathcal{D}_{i}$, from differential equations about $a_{j}(j=0, \ldots, 10)$ to those about $z_{j}(j=1, \ldots, 4)$,

$$
\begin{aligned}
& \mathcal{D}_{1}=\theta_{1}^{3}\left(4 \theta_{1}-2 \theta_{2}+\theta_{3}\right)^{4}\left(\theta_{1}-\theta_{4}\right)-z_{1}\left(-4 \theta_{1}+\theta_{2}\right)^{4}\left(-\theta_{1}+\theta_{4}\right) \prod_{i=1}^{4}\left(-3 \theta_{1}-\theta_{3}-\theta_{4}-i\right), \\
& \mathcal{D}_{2}=\left(-2 \theta_{1}+\theta_{2}\right)\left(\theta_{2}-\theta_{3}\right)-z_{2}\left(2 \theta_{2}-2 \theta_{2}+\theta_{3}\right)^{2} \\
& \mathcal{D}_{3}=\left(\theta_{1}+\theta_{3}-\theta_{4}\right)\left(2 \theta_{1}-2 \theta_{2}+\theta_{3}\right)-z_{3}\left(\theta_{2}-\theta_{3}\right)\left(-4 \theta_{1}-\theta_{3}-1\right), \\
& \mathcal{D}_{4}=\theta_{4}\left(-\theta_{1}+\theta_{4}\right)-z_{4}\left(\theta_{1}+\theta_{3}-\theta_{4}\right)\left(\theta_{1}-\theta_{4}\right)
\end{aligned}
$$

where $\theta_{i}=z_{i} \frac{\partial}{\partial z_{i}}$ 's are the logarithmic derivatives.

\subsubsection{Brane superpotential and disk instantons}

Along the line of 2.1, we solve the differential equations (5.13) at $z_{i} \rightarrow 0$ and identify the mirror maps and superpotential. The fundamental period of $X$ is

$$
\omega_{0}=1+1680 z_{1} z_{2}^{4} z_{3}^{4} z_{4}+32432400 z_{1}^{2} z_{2}^{8} z_{3}^{8} z_{4}^{2}+\mathcal{O}\left(z^{20}\right) .
$$


The single logarithmic solutions are

$$
\begin{aligned}
\omega_{1,1}= & \omega_{0} \log \left(z_{1}\right)-4 z_{2}-6 z_{2}^{2}-\frac{40}{3} z_{2}^{3}+4 z_{2} z_{3}+4 z_{2}^{2} z_{3}+z_{4}+24 z_{1} z_{4}-96 z_{1} z_{2} z_{4}+48 z_{1} z_{2}^{2} z_{4} \\
& -\frac{1}{2} z_{4}^{2}+\frac{1}{3} z_{4}^{3}+\mathcal{O}\left(z^{3}\right) \\
\omega_{1,2}= & \omega_{0} \log \left(z_{2}\right)+2 z_{2}+3 z_{2}^{2}+\frac{20}{3} z_{2}^{3}+z_{3}-2 z_{2} z_{3}-2 z_{2}^{2} z_{3}-\frac{1}{2} z_{3}^{2}+z_{2} z_{3}^{2}+\frac{1}{3} z_{3}^{3}-6 z_{1} z_{4} \\
& +24 z_{1} z_{2} z_{4}+\mathcal{O}\left(z^{3}\right) \\
\omega_{1,3}= & \omega_{0} \log \left(z_{3}\right)-z_{2}-\frac{3}{2} z_{2}^{2}-\frac{10}{3} z_{2}^{3}-\frac{35}{4} z_{2}^{4}-z_{3}+z_{2} z_{3}+z_{2}^{2} z_{3}+2 z_{2}^{3} z_{3}+\frac{1}{2} z_{3}^{2}-z_{2} z_{3}^{2} \\
& -\frac{1}{2} z_{2}^{2} z_{3}^{2}-\frac{1}{3} z_{3}^{3}+z_{2} z_{3}^{3}+\frac{1}{4} z_{3}^{4}+\mathcal{O}\left(z^{4}\right) \\
& \omega_{0} \log \left(z_{4}\right)-z_{4}+\frac{1}{2} z_{4}^{2}-\frac{1}{3} z_{4}^{3}+\frac{1}{4} z_{4}^{4}+\mathcal{O}\left(z^{4}\right)
\end{aligned}
$$

by which the open-closed mirror maps are inverse series of flat coordinates

$$
\begin{aligned}
z_{1}= & q_{1}+4 q_{1} q_{2}+6 q_{1} q_{2}^{2}+4 q_{1} q_{2}^{3}+q_{1} q_{2}^{4}-8 q_{1} q_{2} q_{3}-24 q_{1} q_{2}^{2} q_{3}-q_{1} q_{4}-24 q_{1}^{2} q_{4}-4 q_{1} q_{2} q_{4} \\
& -72 q_{1}^{2} q_{2} q_{4}-6 q_{1} q_{2}^{2} q_{4}+8 q_{1} q_{2} q_{3} q_{4}+24 q_{1}^{2} q_{4}^{2}+\mathcal{O}\left(q^{4}\right) \\
z_{2}= & q_{2}-2 q_{2}^{2}+3 q_{2}^{3}-4 q_{2}^{4}-q_{2} q_{3}+5 q_{2}^{2} q_{3}-13 q_{2}^{3} q_{3}-3 q_{2}^{2} q_{3}^{2}+6 q_{1} q_{2} q_{4}-24 q_{1} q_{2}^{2} q_{4}+\mathcal{O}\left(q^{4}\right) \\
z_{3}= & q_{3}+q_{2} q_{3}+q_{3}^{2}+q_{2}^{2} q_{3}^{2}+q_{3}^{3}+q_{3}^{4}+6 q_{1} q_{2} q_{3} q_{4}+\mathcal{O}\left(q^{4}\right) \\
z_{4}= & q_{4}+q_{4}^{2}+q_{4}^{3}+q_{4}^{4}+\mathcal{O}\left(q^{4}\right)
\end{aligned}
$$

Then we construct a linear combination of double logarithmic solutions and insert the inverse mirror maps,

$$
\mathcal{W}_{\text {brane }}=2 t_{1}^{2}+4 t_{1} t_{4}+2 t_{4}^{2}+\sum_{N} N_{d_{1}, d_{2}, d_{3}, d_{4}} \operatorname{Li}_{2}\left(q_{1}^{d_{1}} q_{2}^{d_{2}} q_{3}^{d_{3}} q_{4}^{d_{4}}\right)
$$

Invariants of the form $N_{\mathrm{m}, \mathrm{m}, \mathrm{m}, \mathrm{n}}$ are summarized in table 18, where the rows and columns are labelled by $m$ and $n$, respectively.

When $a_{7}^{2}=a_{6} a_{8}$, the two individual branes coincide. We obtain a new set of charge vectors,

\begin{tabular}{c|cccccccccc}
\hline & 0 & 1 & 2 & 3 & 4 & 5 & 6 & 8 & 9 & 10 \\
\hline$l_{1}^{c}$ & -3 & 0 & 1 & 1 & 1 & 0 & -2 & 2 & -1 & 1 \\
$l_{2}^{c}$ & -2 & 0 & 0 & 0 & 0 & 2 & 1 & -1 & 0 & 0 \\
$l_{3}^{c}$ & -1 & 1 & 0 & 0 & 0 & 0 & 0 & 0 & 1 & -1 \\
\hline
\end{tabular}

by which the new complex structure moduli space coordinates are

$$
z_{1}^{c}=\frac{a_{2} a_{3} a_{4} a_{8}^{2} a_{10}}{a_{0}^{3} a_{6}^{2} a_{9}}, \quad z_{2}^{c}=\frac{a_{5}^{2} a_{6}}{a_{0}^{2} a_{8}}, \quad z_{3}^{c}=\frac{a_{1} a_{9}}{a_{0} a_{10}}
$$

Similar to the separate case, the superpotential is constructed as linear combination of double logarithmic solutions and Ooguri-Vafa invariants are extracted in table 18. 


\section{Summary and conclusions}

In this work, we calculate the superpotentials in $d=4 \mathrm{~N}=1$ supersymmetric field theories arising from IIA D6-branes wrapping special Lagrangian three cycles of Calabi-Yau threefold. The special Lagrangian three-cycles with non-trivial topology are mirror to obstructed rational curves, which correspond to the brane excitation about the supersymmetric minimum. We consider a five brane wrapping a rational curve that coincides with a toric curve $S$ at certain locus of the deformation space $\mathcal{M}(S) . S$ is described by the intersection of two divisors $D_{1} \cap D_{2}$ and its unobstructed deformation space match with the obstructed deformation space of the rational curve wrapped by the five brane. After blowing up, the toric curve $S$ is replaced by a exceptional divisor $E$ without introducing new degree of freedom. All the complex structure moduli and brane moduli are embedded into the complex moduli space of the blow-up new manifold, given as the complete intersection in the projective bundle $\mathcal{W}=\mathbb{P}\left(\mathcal{O}\left(D_{1}\right) \oplus \mathcal{O}\left(D_{2}\right)\right)$.

From observation on the defining equation of $X^{\prime}$, we obtain the Picard-Fuchs equations that annihilate the period matrix defined by the natural pairing between the elements of relative homology $H_{*}(X, S)$ and cohomology $H^{*}(X, S)$. Via GKZ system of $X^{\prime}$, the system of Picard-Fuchs equations are solved at $z_{i} \rightarrow 0$. The single logarithmic solutions are interpreted as mirror maps and specific linear combinations of double logarithmic solutions are the B-brane superpotentials. Using multi-cover formula and inverse mirror maps, the Ooguri-Vafa invariants are extracted from A-model side and interpreted as counting disk instantons, i.e. holomorphic disks with boundary in a nontrivial homology class on a special Lagrangian submanifold. It would be interesting to directly extract these invariants on the A-model side directly by adequate localization techniques.

\section{Acknowledgments}

This work is supported by NSFC(11475178).

\section{A Blow-up geometry of $X_{[4,4]}^{(112 \mid 112)}$ and $X_{[6,6]}^{(123 \mid 123)}$}

\section{A.1 $X_{[4,4]}^{(112 \mid 112)}$}

Polyhedron vertices and charge vectors associated to A-model manifold of $X^{*}$ and A-branes on it (table 6): the GKZ system of $X$ as follows

$$
\begin{aligned}
& \mathcal{Z}_{0}=\sum_{i=0}^{6} \vartheta_{i}+1, \quad \mathcal{Z}_{1}=-\vartheta_{1}+\vartheta_{2}, \quad \mathcal{Z}_{2}=-2 \vartheta_{1}+\vartheta_{3}, \\
& \mathcal{Z}_{3}=-\vartheta_{1}+\vartheta_{4}, \quad \mathcal{Z}_{4}=-2 \vartheta_{1}+\vartheta_{5}, \quad \mathcal{Z}_{5}=-2 \vartheta_{1}+\vartheta_{6} \\
& \mathcal{L}_{1}=\frac{\partial}{\partial a_{1}} \frac{\partial}{\partial a_{2}}\left(\frac{\partial}{\partial a_{3}}\right)^{2} \frac{\partial}{\partial a_{4}} \frac{\partial}{\partial a_{5}}\left(\frac{\partial}{\partial a_{6}}\right)^{2}-\left(\frac{\partial}{\partial a_{0,1}}\right)^{3}\left(\frac{\partial}{\partial a_{0,2}}\right)^{3}
\end{aligned}
$$




\begin{tabular}{|c|c|c|c|c|c|c|c|c|c|}
\hline & \multicolumn{5}{|c|}{$\Delta^{*}$} & $l$ & & $\hat{l}^{1}$ & $\hat{l}^{2}$ \\
\hline$v_{0,1}^{*}$ & 0 & 0 & 0 & 0 & 0 & -4 & $x_{1} x_{2} x_{3}$ & -1 & -1 \\
\hline$v_{0,2}^{*}$ & 0 & 0 & 0 & 0 & 0 & -4 & $x_{4} x_{5} x_{6}$ & 0 & 0 \\
\hline$v_{1}^{*}$ & -1 & -2 & -1 & -1 & -2 & 1 & $x_{1}^{4}$ & 0 & 0 \\
\hline$v_{2}^{*}$ & 1 & 0 & 0 & 0 & 0 & 1 & $x_{2}^{4}$ & 1 & 0 \\
\hline$v_{3}^{*}$ & 0 & 1 & 0 & 0 & 0 & 2 & $x_{3}^{4}$ & 0 & 1 \\
\hline$v_{4}^{*}$ & 0 & 0 & 1 & 0 & 0 & 1 & $x_{4}^{4}$ & 0 & 0 \\
\hline$v_{5}^{*}$ & 0 & 0 & 0 & 1 & 0 & 1 & $x_{5}^{4}$ & 0 & 0 \\
\hline$v_{6}^{*}$ & 0 & 0 & 0 & 0 & 1 & 2 & $x_{6}^{2}$ & 0 & 0 \\
\hline
\end{tabular}

Table 6. Toric data of A-model manifold.

After blowing up $X$ along the curve specified by $\hat{l}^{1}, \hat{l}^{2}$, the GKZ system of blow-up manifold $X^{\prime}$ :

$\mathcal{Z}_{0}^{\prime}=\sum_{i=0}^{6} \vartheta_{i}+1, \quad \mathcal{Z}_{1}^{\prime}=\sum_{i=7}^{10} \vartheta_{i}, \quad \mathcal{Z}_{2}^{\prime}=-\vartheta_{1}+\vartheta_{2}-\vartheta_{8}+\vartheta_{10}, \quad \mathcal{Z}_{3}^{\prime}=-2 \vartheta_{1}+\vartheta_{3}-2 \vartheta_{8}$,

$\mathcal{Z}_{4}^{\prime}=-\vartheta_{1}+\vartheta_{4}-\vartheta_{8}, \quad \mathcal{Z}_{5}^{\prime}=-\vartheta_{1}+\vartheta_{5}-\vartheta_{8}, \quad \mathcal{Z}_{6}^{\prime}=-2 \vartheta_{1}+\vartheta_{6}-2 \vartheta_{8}, \quad \mathcal{Z}_{7}^{\prime}=-\vartheta_{7}-\vartheta_{8}+\vartheta_{9}+\vartheta_{10}$

$\mathcal{L}_{1}^{\prime}=\frac{\partial}{\partial a_{1}} \frac{\partial}{\partial a_{2}}\left(\frac{\partial}{\partial a_{3}}\right)^{2} \frac{\partial}{\partial a_{4}} \frac{\partial}{\partial a_{5}}\left(\frac{\partial}{\partial a_{6}}\right)^{2}-\left(\frac{\partial}{\partial a_{0,1}}\right)^{3}\left(\frac{\partial}{\partial a_{0,2}}\right)^{3}$,

$\mathcal{L}_{2}^{\prime}=\frac{\partial}{\partial a_{1}} \frac{\partial}{\partial a_{7}}-\frac{\partial}{\partial a_{0,1}} \frac{\partial}{\partial a_{8}}, \quad \mathcal{L}_{3}^{\prime}=\frac{\partial}{\partial a_{2}} \frac{\partial}{\partial a_{9}}-\frac{\partial}{\partial a_{0,1}} \frac{\partial}{\partial a_{10}}$

Toric data for the enhanced polyhedron associated to the $X^{\prime}$ :

\begin{tabular}{|c|c|c|c|c|c|c|c|c|c|c|c|c|}
\hline & \multicolumn{8}{|c|}{$\Delta^{\prime}$} & & $l_{2}^{\prime}$ & $l_{3}^{\prime}$ & \\
\hline$v_{0,1}^{\prime}$ & 1 & 0 & 0 & 0 & 0 & 0 & 0 & 0 & -1 & -1 & -1 & $w_{0,1}^{\prime}=x_{1} x_{2} x_{3}$ \\
\hline$v_{0,2}^{\prime}$ & 1 & 0 & 0 & 0 & 0 & 0 & 0 & 0 & -4 & 0 & 0 & $w_{0,2}^{\prime}=x_{4} x_{5} x_{6}$ \\
\hline$v_{1}^{\prime}$ & 1 & 0 & -1 & -2 & -1 & -1 & -2 & 0 & -1 & 1 & 0 & $w_{1}^{\prime}=x_{1}^{4}$ \\
\hline$v_{2}^{\prime}$ & 1 & 0 & 1 & 0 & 0 & 0 & 0 & 0 & 0 & 0 & 1 & $w_{2}^{\prime}=x_{2}^{4}$ \\
\hline$v_{3}^{\prime}$ & 1 & 0 & 0 & 1 & 0 & 0 & 0 & 0 & 2 & 0 & 0 & $w_{3}^{\prime}=x_{3}^{4}$ \\
\hline$v_{4}^{\prime}$ & 1 & 0 & 0 & 0 & 1 & 0 & 0 & 0 & 1 & 0 & 0 & $w_{4}^{\prime}=x_{4}^{2}$ \\
\hline$v_{5}^{\prime}$ & 1 & 0 & 0 & 0 & 0 & 1 & 0 & 0 & 1 & 0 & 0 & $w_{5}^{\prime}=x_{5}^{4}$ \\
\hline$v_{6}^{\prime}$ & 1 & 0 & 0 & 0 & 0 & 0 & 1 & 0 & 2 & 0 & 0 & $w_{6}^{\prime}=x_{6}^{4}$ \\
\hline$v_{7}^{\prime}$ & 0 & 1 & 0 & 0 & 0 & 0 & 0 & -1 & -2 & 1 & 0 & $w_{7}^{\prime}=y_{1} w_{0}^{\prime}$ \\
\hline$v_{8}^{\prime}$ & 0 & 1 & -1 & -2 & -1 & -1 & -2 & -1 & 2 & -1 & 0 & $w_{8}^{\prime}=y_{1} w_{2}^{\prime}$ \\
\hline$v_{9}^{\prime}$ & 0 & 1 & 0 & 0 & 0 & 0 & 0 & 1 & -1 & 0 & 1 & $w_{9}^{\prime}=y_{2} w_{0}^{\prime}$ \\
\hline$v_{10}^{\prime}$ & 0 & 1 & 1 & 0 & 0 & 0 & 0 & 1 & 1 & 0 & -1 & $w_{10}^{\prime}=y_{2} w_{3}^{\prime}$ \\
\hline
\end{tabular}


Picard-Fuchs operators from above table:

$$
\begin{aligned}
\mathcal{D}_{1}= & \left(2 \theta_{1}\right)^{2}\left(\theta_{1}\right)^{2}\left(2 \theta_{1}\right)^{2}\left(2 \theta_{1}-\theta_{2}\right)^{2}\left(\theta_{1}-\theta_{3}\right) \\
& -z_{1}\left(-\theta_{1}+\theta_{2}\right)\left(-2 \theta_{1}+\theta_{2}\right)^{2}\left(-\theta_{1}+\theta_{3}\right)\left(-\theta_{1}-\theta_{2}-\theta_{3}-1\right) \prod_{i=1}^{4}\left(-4 \theta_{1}-i\right) \\
\mathcal{D}_{2}= & \left(-\theta_{1}+\theta_{2}\right)\left(-2 \theta_{1}+\theta_{2}\right)-z_{2}\left(-\theta_{1}-\theta_{2}-\theta_{3}-1\right)\left(2 \theta_{1}-\theta_{2}\right) \\
\mathcal{D}_{3}= & \theta_{3}\left(-\theta_{1}+\theta_{3}\right)-z_{3}\left(-\theta_{1}-\theta_{2}-\theta_{3}-1\right)\left(\theta_{1}-\theta_{3}\right)
\end{aligned}
$$

Solving above equations by GKZ-system, the unique power series solution, as well as the fundamental period of $X$, is

$$
\begin{aligned}
\omega_{0}= & 1+144 z_{1} z_{2}^{2} z_{3}+176400 z_{1}^{2} z_{2}^{4} z_{3}^{2}+341510400 z_{1}^{3} z_{2}^{6} z_{3}^{3}+811620810000 z_{1}^{4} z_{2}^{8} z_{3}^{4} \\
& +\mathcal{O}\left(z^{16}\right)
\end{aligned}
$$

and four single logarithmic solutions

$$
\begin{aligned}
\omega_{1,1}= & \omega_{0} \log \left(z_{1}\right)+2 z_{2}-z_{2}^{2}-36 z_{1} z_{2}^{2}+\frac{2}{3} z_{2}^{3}-\frac{1}{2} z_{2}^{4}+3150 z_{1}^{2} z_{2}^{4}+\frac{2}{5} z_{2}^{5}+z_{3} \\
& -72 z_{1} z_{2} z_{3}+492 z_{1} z_{2}^{2} z_{3}+720 z_{1} z_{2}^{3} z_{3}-\frac{1}{2} z_{3}^{2}+360 z_{1} z_{2}^{2} z_{3}^{2}+\frac{1}{3} z_{3}^{3}-\frac{1}{4} z_{3}^{4} \\
& +\frac{1}{5} z_{3}^{5}+\mathcal{O}\left(z^{5}\right) \\
\omega_{1,2}= & \omega_{0} \log \left(z_{2}\right)-z_{2}+\frac{1}{2} z_{2}^{2}-\frac{1}{3} z_{2}^{3}+\frac{1}{4} z_{2}^{4}-\frac{1}{5} z_{2}^{5}+36 z_{1} z_{2} z_{3}+156 z_{1} z_{2}^{2} z_{3} \\
& -360 z_{1} z_{2}^{3} z_{3}+\mathcal{O}\left(z^{5}\right) \\
\omega_{1,3}= & \omega_{0} \log \left(z_{3}\right)+36 z_{1} z_{2}^{2}-z_{3}+156 z_{1} z_{2}^{2} z_{3}+\frac{1}{2} z_{3}^{2}-360 z_{1} z_{2}^{2} z_{3}^{2}-\frac{1}{3} z 3^{3} \\
& +360 z_{1} z_{2}^{2} z_{3}^{3}+\frac{1}{4} z_{3}^{4}-\frac{1}{5} z 3^{5}+\mathcal{O}\left(z^{5}\right)
\end{aligned}
$$

Upon the fundamental period and single logarithmic solutions, the inverse mirror maps are

$$
\begin{aligned}
z_{1}= & q_{1}-2 q_{1} q_{2}+q_{1} q_{2}^{2}+36 q_{1}^{2} q_{2}^{2}-72 q_{1}^{2} q_{2}^{3}-q_{1} q_{3}+2 q_{1} q_{2} q_{3}+72 q_{1}^{2} q_{2} q_{3}-q_{1} q_{2}^{2} q_{3} \\
& \quad-672 q_{1}^{2} q_{2}^{2} q_{3}-72 q_{1}^{2} q_{2} q_{3}^{2}+\mathcal{O}\left(q^{5}\right) \\
z_{2}= & q_{2}+q_{2}^{2}+q_{2}^{3}+q_{2}^{4}+q_{2}^{5}-36 q_{1} q_{2}^{2} q_{3}-192 q_{1} q_{2}^{3} q_{3}+\mathcal{O}\left(q^{5}\right) \\
z_{3}= & q_{3}-36 q_{1} q_{2}^{2} q_{3}+q_{3}^{2}-192 q_{1} q_{2}^{2} q_{3}^{2}+q_{3}^{3}+\mathcal{O}\left(q^{5}\right)
\end{aligned}
$$

The brane superpotential as linear combination of double logarithmic solutions is

$$
\mathcal{W}_{\text {brane }}=4 t_{1} t_{2}+6 t_{2}^{2}+4 t_{2} t_{3}+\sum_{N} N_{d_{1}, d_{2}, d_{3}} \operatorname{Li}_{2}\left(q_{1}^{d_{1}} q_{2}^{d_{2}} q_{3}^{d_{3}}\right)
$$

and the disk instantons are presented in table 16. 


\begin{tabular}{|c|c|c|c|c|c|c|c|c|c|}
\hline & \multicolumn{5}{|c|}{$\Delta^{*}$} & $l$ & & $\hat{l}^{1}$ & $\hat{l}^{2}$ \\
\hline$v_{0,1}^{*}$ & 0 & 0 & 0 & 0 & 0 & -6 & $x_{1} x_{2} x_{3}$ & 0 & 0 \\
\hline$v_{0,2}^{*}$ & 0 & 0 & 0 & 0 & 0 & -6 & $x_{4} x_{5} x_{6}$ & 0 & 0 \\
\hline$v_{1}^{*}$ & -2 & -3 & -1 & -2 & -3 & 1 & $x_{1}^{6}$ & -1 & -1 \\
\hline$v_{2}^{*}$ & 1 & 0 & 0 & 0 & 0 & 2 & $x_{2}^{3}$ & 1 & 0 \\
\hline$v_{3}^{*}$ & 0 & 1 & 0 & 0 & 0 & 3 & $x_{3}^{2}$ & 0 & 1 \\
\hline$v_{4}^{*}$ & 0 & 0 & 1 & 0 & 0 & 1 & $x_{4}^{6}$ & 0 & 0 \\
\hline$v_{5}^{*}$ & 0 & 0 & 0 & 1 & 0 & 2 & $x_{5}^{3}$ & 0 & 0 \\
\hline$v_{6}^{*}$ & 0 & 0 & 0 & 0 & 1 & 3 & $x_{6}^{2}$ & 0 & 0 \\
\hline
\end{tabular}

Table 7. Toric Data of A-model side.

\section{A.2 $X_{[6,6]}^{(123 \mid 123)}$}

Polyhedron vertices and charge vectors A-model manifold $X^{*}$ and A-branes on it (table 7): GKZ system of $X$ as follows

$$
\begin{aligned}
\mathcal{Z}_{0} & =\sum_{i=0}^{6} \vartheta_{i}+1, \quad \mathcal{Z}_{1}=-2 \vartheta_{1}+\vartheta_{2}, \quad \mathcal{Z}_{2}=-3 \vartheta_{1}+\vartheta_{3} \\
\mathcal{Z}_{3} & =-\vartheta_{1}+\vartheta_{4}, \quad \mathcal{Z}_{4}=-2 \vartheta_{1}+\vartheta_{5}, \quad \mathcal{Z}_{5}=-3 \vartheta_{5}+\vartheta_{6} \\
\mathcal{L}_{1} & =\left(\frac{\partial}{\partial a_{1}}\right)\left(\frac{\partial}{\partial a_{2}}\right)^{2}\left(\frac{\partial}{\partial a_{3}}\right)^{3}\left(\frac{\partial}{\partial a_{4}}\right)\left(\frac{\partial}{\partial a_{5}}\right)^{2}\left(\frac{\partial}{\partial a_{6}}\right)^{3}-\left(\frac{\partial}{\partial a_{0,1}}\right)^{6}\left(\frac{\partial}{\partial a_{0,2}}\right)^{6}
\end{aligned}
$$

After blowing up along the curve specified by $\hat{l}^{1}, \hat{l}^{2}$, the GKZ system of blow-up manifold $X^{\prime}$ :

$$
\begin{aligned}
& \mathcal{Z}_{0}^{\prime}=\sum_{i=0}^{6} \vartheta_{i}+1, \quad \mathcal{Z}_{1}^{\prime}=\sum_{i=7}^{10} \vartheta_{i}, \quad \mathcal{Z}_{2}^{\prime}=-2 \vartheta_{1}+\vartheta_{2}-2 \vartheta_{7}+\vartheta_{8}-2 \vartheta_{9}, \\
& \mathcal{Z}_{3}^{\prime}=-3 \vartheta_{1}+\vartheta_{3}-3 \vartheta_{7}-3 \vartheta_{9}+\vartheta_{10}, \quad \mathcal{Z}_{4}^{\prime}=-\vartheta_{1}+\vartheta_{4}-\vartheta_{7}-\vartheta_{9}, \quad \mathcal{Z}_{5}^{\prime}=-2 \vartheta_{1}+\vartheta_{5}-2 \vartheta_{7}-2 \vartheta_{9}, \\
& \mathcal{Z}_{6}^{\prime}=-3 \vartheta_{1}+\vartheta_{6}-3 \vartheta_{7}-3 \vartheta_{9}, \quad \mathcal{Z}_{7}^{\prime}=-\vartheta_{7}-\vartheta_{8}+\vartheta_{9}+\vartheta_{10} \\
& \mathcal{L}_{1}^{\prime}=\left(\frac{\partial}{\partial a_{1}}\right)\left(\frac{\partial}{\partial a_{2}}\right)^{2}\left(\frac{\partial}{\partial a_{3}}\right)^{3}\left(\frac{\partial}{\partial a_{4}}\right)\left(\frac{\partial}{\partial a_{5}}\right)^{2}\left(\frac{\partial}{\partial a_{6}}\right)^{3}-\left(\frac{\partial}{\partial a_{0,1}}\right)^{6}\left(\frac{\partial}{\partial a_{0,2}}\right)^{6}, \\
& \mathcal{L}_{2}^{\prime}=\frac{\partial}{\partial a_{1}} \frac{\partial}{\partial a_{8}}-\frac{\partial}{\partial a_{3}} \frac{\partial}{\partial a_{7}}, \quad \mathcal{L}_{3}^{\prime}=\frac{\partial}{\partial a_{1}} \frac{\partial}{\partial a_{10}}-\frac{\partial}{\partial a_{2}} \frac{\partial}{\partial a_{9}}
\end{aligned}
$$


Toric data for the enhanced polyhedron associated to the $X^{\prime}$ 's mirror manifold:

\begin{tabular}{|c|c|c|c|c|c|c|c|c|c|c|c|c|}
\hline & \multicolumn{8}{|c|}{$\Delta^{\prime}$} & \multicolumn{4}{|c|}{$\begin{array}{lll}l_{1}^{\prime} & l_{2}^{\prime} & l_{3}^{\prime}\end{array}$} \\
\hline$v_{0,1}^{\prime}$ & 1 & 0 & 0 & 0 & 0 & 0 & 0 & 0 & -6 & 0 & 0 & $w_{0,1}^{\prime}=x_{1} x_{2} x_{3}$ \\
\hline$v_{0,2}^{\prime}$ & 1 & 0 & 0 & 0 & 0 & 0 & 0 & 0 & -6 & 0 & 0 & $w_{0,2}^{\prime}=x_{4} x_{5} x_{6}$ \\
\hline$v_{1}^{\prime}$ & 1 & 0 & -2 & -3 & -1 & -2 & -3 & 0 & -2 & 1 & 1 & $w_{1}^{\prime}=x_{1}^{6}$ \\
\hline$v_{2}^{\prime}$ & 1 & 0 & 1 & 0 & 0 & 0 & 0 & 0 & 3 & 0 & -1 & $w_{2}^{\prime}=x_{2}^{3}$ \\
\hline$v_{3}^{\prime}$ & 1 & 0 & 0 & 1 & 0 & 0 & 0 & 0 & 5 & -1 & 0 & $w_{3}^{\prime}=x_{3}^{2}$ \\
\hline$v_{4}^{\prime}$ & 1 & 0 & 0 & 0 & 1 & 0 & 0 & 0 & 1 & 0 & 0 & $w_{4}^{\prime}=x_{4}^{6}$ \\
\hline$v_{5}^{\prime}$ & 1 & 0 & 0 & 0 & 0 & 1 & 0 & 0 & 2 & 0 & 0 & $w_{5}^{\prime}=x_{5}^{3}$ \\
\hline$v_{6}^{\prime}$ & 1 & 0 & 0 & 0 & 0 & 0 & 1 & 0 & 3 & 0 & 0 & $w_{6}^{\prime}=x_{6}^{2}$ \\
\hline$v_{7}^{\prime}$ & 0 & 1 & -2 & -3 & -1 & -2 & -3 & -1 & 1 & 0 & -1 & $w_{7}^{\prime}=y_{1} w_{0}^{\prime}$ \\
\hline$v_{8}^{\prime}$ & 0 & 1 & 1 & 0 & 0 & 0 & 0 & -1 & -1 & 0 & 1 & $w_{8}^{\prime}=y_{1} w_{2}^{\prime}$ \\
\hline$v_{9}^{\prime}$ & 0 & 1 & -2 & -3 & -1 & -2 & -3 & 1 & 2 & -1 & 0 & $w_{9}^{\prime}=y_{2} w_{0}^{\prime}$ \\
\hline$v_{10}^{\prime}$ & 0 & 1 & 0 & 1 & 0 & 0 & 0 & 1 & -2 & 1 & 0 & $w_{10}^{\prime}=y_{2} w_{3}^{\prime}$ \\
\hline
\end{tabular}

Picard-Fuchs equations from above table:

$$
\begin{aligned}
\mathcal{D}_{1}= & \left(3 \theta_{1}-\theta_{3}\right)^{3}\left(5 \theta_{1}-\theta_{2}\right)^{5} \theta_{1}\left(2 \theta_{1}\right)^{2}\left(3 \theta_{1}\right)^{3}\left(2 \theta_{1}-\theta_{2}\right)^{2}\left(\theta_{1}-\theta_{3}\right) \\
& -z_{1}\left(-2 \theta_{1}+\theta_{2}+\theta_{3}\right)^{2}\left(-2 \theta_{1}+\theta_{2}\right)^{2}\left(-\theta_{1}+\theta_{3}\right) \prod_{i=1}^{6} \prod_{j=1}^{6}\left(-6 \theta_{1}-i\right)\left(-6 \theta_{1}-j\right) \\
\mathcal{D}_{2}= & \left(-2 \theta_{1}+\theta_{2}+\theta_{3}\right)\left(-2 \theta_{1}+\theta_{2}\right)-z_{2}\left(5 \theta_{1}-\theta_{2}\right)\left(2 \theta_{1}-\theta_{2}\right) \\
\mathcal{D}_{3}= & \left(-2 \theta_{1}+\theta_{2}+\theta_{3}\right)\left(-\theta_{1}+\theta_{3}\right)-z_{3}\left(3 \theta_{1}-\theta_{3}\right)\left(\theta_{1}-\theta_{3}\right)
\end{aligned}
$$

Soling above equations by GKZ-system, the unique power series solution, as well as the fundamental period of $X$, is

$$
\begin{aligned}
\omega_{0}= & 1+3600 z_{1} z_{2}^{2} z_{3}+192099600 z_{1}^{2} z_{2}^{4} z_{3}^{2}+16679709446400 z_{1}^{3} z_{2}^{6} z_{3}^{3} \\
& +1791735431214128400 z_{1}^{4} z_{2}^{8} z_{3}^{4}+\mathcal{O}\left(z^{16}\right)
\end{aligned}
$$

and the single logarithmic solutions are

$$
\begin{aligned}
\omega_{1,1}= & \omega_{0} \log \left(z_{1}\right)-1200 z_{1} z_{2}^{2}-1800 z_{1} z_{2} z_{3}+29640 z_{1} z_{2}^{2} z_{3}+10800 z_{1} z_{2}^{3} z_{3} \\
& +3600 z_{1} z_{2}^{2} z_{3}^{2}+\mathcal{O}\left(z^{5}\right) \\
\omega_{1,2}= & \omega_{0} \log \left(z_{2}\right)+900 z_{1} z_{2} z_{3}+3000 z_{1} z_{2}^{2} z_{3}-5400 z_{1} z_{2}^{3} z_{3}+\mathcal{O}\left(z^{5}\right) \\
\omega_{1,3}= & \omega_{0} \log \left(z_{3}\right)+1200 z_{1} z_{2}^{2}+1800 z_{1} z_{2}^{2} z_{3}-3600 z_{1} z_{2}^{2} z_{3}^{2}+\mathcal{O}\left(z^{5}\right)
\end{aligned}
$$

By the fundamental period and single logarithmic solutions, the inverse mirror maps are

$$
\begin{aligned}
& z_{1}=q_{1}+1200 q_{1}^{2} q_{2}^{2}-4243320 q_{1}^{3} q_{2}^{4}+1800 q_{1}^{2} q_{2} q_{3}-29640 q_{1}^{2} q_{2}^{2} q_{3}+\mathcal{O}\left(q^{5}\right) \\
& z_{2}=q_{2}-900 q_{1} q_{2}^{2} q_{3}-3000 q_{1} q_{2}^{3} q_{3}+\mathcal{O}\left(q^{5}\right) \\
& z_{3}=q_{3}-1200 q_{1} q_{2}^{2} q_{3}+5683320 q_{1}^{2} q_{2}^{4} q_{3}-1800 q_{1} q_{2}^{2} q_{3}^{2}+\mathcal{O}\left(q^{5}\right)
\end{aligned}
$$


The brane superpotential is

$$
\mathcal{W}_{\text {brane }}=4 t_{1} t_{2}+6 t_{2}^{2}+4 t_{2} t_{3}+\sum_{N} N_{d_{1}, d_{2}, d_{3}} \operatorname{Li}_{2}\left(q_{1}^{d_{1}} q_{2}^{d_{2}} q_{3}^{d_{3}}\right)
$$

and the disk instantons are presented in table 16 


\section{B Ooguri-Vafa invariants for two closed and two open moduli}

\section{B.1 Ooguri-Vafa invariants for $X_{9}^{(1,1,3,3,3)}$}

\begin{tabular}{|c|cccccc|}
\hline \multicolumn{7}{|c|}{$\mathrm{k}=0$ : Ooguri-Vafa Invariants } \\
$N_{0, m, 0, m+n}$ & $n=1$ & 2 & 3 & 4 & 5 & 6 \\
\hline$m=0$ & 3 & 0 & 0 & 0 & 0 & 0 \\
1 & -3 & -3 & -3 & -3 & -3 & -3 \\
2 & 15 & 21 & 27 & 36 & 45 & 57 \\
3 & -120 & -183 & -279 & -420 & -618 & $*$ \\
4 & 1179 & 1944 & 3210 & 5250 & $*$ & $*$ \\
5 & -13572 & -22983 & -39771 & $*$ & $*$ & $*$ \\
\hline
\end{tabular}

\begin{tabular}{|c|cccccc|}
\hline \multicolumn{7}{|c|}{$\mathrm{k}=1$ : Ooguri-Vafa Invariants } \\
$N_{k, m, k, m+n}$ \\
\hline$N_{1, m, 1, m+n}$ & $n=1$ & 2 & 3 & 4 & 5 & 6 \\
\hline 1 & 27 & 0 & 0 & 0 & 0 & 0 \\
2 & 90 & 90 & 90 & 90 & 90 & 90 \\
3 & -684 & -954 & -1314 & -1764 & -2304 & -2934 \\
4 & 7470 & 11736 & 18486 & 28620 & 43218 & $*$ \\
5 & -94644 & -158022 & -267768 & -450270 & $*$ & $*$ \\
\end{tabular}

\begin{tabular}{|c|cccccc|}
\hline \multicolumn{7}{|c|}{$\mathrm{k}=2$ : Ooguri-Vafa Invariants } \\
$N_{2, m, 2, m+n}$ & $n=1$ & 2 & 3 & 4 & 5 & 6 \\
\hline$m=0$ & 81 & 108 & 0 & 0 & 0 & 0 \\
1 & -1539 & -1377 & -1377 & -1377 & -1377 & -1377 \\
2 & 13554 & 19728 & 29079 & 41112 & 55917 & 73404 \\
3 & -204120 & -333612 & -553878 & 898587 & -1412154 & $*$ \\
4 & 3351969 & 5781384 & 10209969 & 17848764 & $*$ & $*$ \\
5 & -56886543 & -101222919 & -185715828 & $*$ & $*$ & $*$ \\
\hline
\end{tabular}

\begin{tabular}{|c|cccccc|}
\hline \multicolumn{7}{|c|}{$\mathrm{k}=3$ : Ooguri-Vafa Invariants } \\
$N_{k, m, k, m+n}$ \\
\hline$m=0$ & $n=1$ & 2 & 3 & 4 & 5 & 6 \\
1 & 18150 & 16764 & 14796 & 14796 & 14796 & 14796 \\
2 & -132492 & -221262 & -370224 & -580212 & -864306 & -1168506 \\
3 & 3063876 & 5345640 & 9647424 & 16885548 & 28230822 & $*$ \\
4 & -68640885 & -123720696 & -231991668 & -428397570 & $*$ & $*$ \\
5 & 1903950048 & 2812011138 & 5315645308 & $*$ & $*$ & $*$ \\
\hline
\end{tabular}

Table 8. Ooguri-Vafa Invariants $N_{k, m, k, m+n}$ for $X_{9}^{(1,1,1,3,3)}$ with brane I at large volume. $k$ and $m$ label the class $t_{1}, t_{2}$ of $X_{9}$ and $n$ labels the brane winding. Blue entries agree with table 5 of [8] and entries $*$ exceed in this table the order of our calculation. 


\begin{tabular}{|c|cccccc|}
\hline \multicolumn{7}{|c|}{$\mathrm{k}=0$ : Ooguri-Vafa Invariants $N_{k, m, k, m+n}$} \\
\hline$N_{0, m, 0, m+n}$ & $n=1$ & 2 & 3 & 4 & 5 & 6 \\
\hline$m=0$ & 54 & 0 & 0 & 0 & 0 & 0 \\
1 & 54 & -18 & 0 & 0 & 0 & 0 \\
2 & 36 & 0 & 0 & 0 & 0 & 0 \\
3 & 54 & 0 & 0 & 0 & 0 & $*$ \\
4 & 54 & -18 & 0 & 0 & $*$ & $*$ \\
5 & 36 & 0 & 0 & $*$ & $*$ & $*$ \\
\hline
\end{tabular}

\begin{tabular}{|c|cccccc|}
\hline \multicolumn{7}{|c|}{$\mathrm{k}=1$ : Ooguri-Vafa Invariants $N_{k, m, k, m+n}$} \\
\hline$N_{1, m, 1, m+n}$ & $n=1$ & 2 & 3 & 4 & 5 & 6 \\
\hline$m=0$ & 0 & 0 & 0 & 0 & 0 & 0 \\
1 & -108 & 36 & 0 & 0 & 0 & 0 \\
2 & 2772 & -1026 & 0 & 18 & 0 & 0 \\
3 & 243756 & -193050 & 100548 & -33588 & 6696 & $*$ \\
4 & 2947320 & -2801070 & 2212272 & -1340010 & $*$ & $*$ \\
5 & 23798376 & -22631562 & 19965852 & $*$ & $*$ & $*$ \\
\hline
\end{tabular}

\begin{tabular}{|c|cccccc|}
\hline \multicolumn{7}{|c|}{$\mathrm{k}=2$ : Ooguri-Vafa Invariants $N_{k, m, k, m+n}$} \\
\hline$N_{2, m, 2, m+n}$ & $n=1$ & 2 & 3 & 4 & 5 & 6 \\
\hline$m=0$ & 0 & 0 & 0 & 0 & 0 & 0 \\
1 & 270 & -90 & 0 & 0 & 0 & 0 \\
2 & -11160 & 4104 & 0 & -72 & 0 & 0 \\
3 & 174960 & -74358 & 162 & 2916 & 0 & $*$ \\
4 & -7067304 & 6235344 & -4430376 & 2634444 & $*$ & $*$ \\
5 & 92080530 & -71321472 & 602803296 & $*$ & $*$ & $*$ \\
\hline
\end{tabular}

\begin{tabular}{|c|cccccc|}
\hline \multicolumn{7}{|c|}{$\mathrm{k}=3$ : Ooguri-Vafa Invariants $N_{k, m, k, m+n}$} \\
\hline$N_{3, m, 3, m+n}$ & $n=1$ & 2 & 3 & 4 & 5 & 6 \\
\hline$m=0$ & 0 & 0 & 0 & 0 & 0 & 0 \\
1 & 0 & 0 & 0 & 0 & 0 & 0 \\
2 & 0 & 0 & 0 & 0 & 0 & 0 \\
3 & 0 & 0 & 12 & 0 & 0 & $*$ \\
4 & 0 & 0 & 0 & 0 & $*$ & $*$ \\
5 & 0 & 194724 & -541296 & $*$ & $*$ & $*$ \\
\hline
\end{tabular}

Table 9. Ooguri-Vafa Invariants $N_{k, m, k, m+n}$ for $X_{9}^{(1,1,1,3,3)}$ with brane II at large volume. $k$ and $m$ label the class $t_{1}, t_{2}$ of $X_{9}$ and $n$ labels the brane winding. Blue entries agree with table 6 of [8] and entries $*$ exceed in this table the order of our calculation. 


\begin{tabular}{|c|c|c|c|c|c|c|}
\hline \multicolumn{7}{|c|}{$\mathrm{k}=1:$ Ooguri-Vafa Invariants $N_{k, m+n, k, n}$} \\
\hline$N_{1, m+n, 1, n}$ & $n=0$ & 1 & 2 & 3 & 4 & 5 \\
\hline$m=1$ & 72 & -1728 & -80460 & -1075140 & -9482724 & -65006280 \\
\hline 2 & -36 & 17280 & 340092 & 3488940 & 25895214 & 156528234 \\
\hline 3 & -1224 & -64800 & -977832 & -8913456 & -61353288 & -350009424 \\
\hline 4 & 5508 & 176688 & 2301588 & 19441008 & 127166868 & $*$ \\
\hline 5 & -15336 & -398304 & -4742280 & -38004912 & $*$ & $*$ \\
\hline 6 & 33948 & 787968 & 8853948 & $*$ & $*$ & $*$ \\
\hline
\end{tabular}

\begin{tabular}{|c|cccccc|}
\hline \multicolumn{7}{|c|}{$\mathrm{k}=2$ : Ooguri-Vafa Invariants $N_{k, m+n, k, n}$} \\
\hline$N_{2, m+n, 2, n}$ & $n=0$ & 1 & 2 & 3 & 4 & 5 \\
\hline$m=1$ & -180 & 7020 & -97686 & 2643372 & -37415520 & -6097896648 \\
2 & 108 & -5832 & -588276 & 3924954 & 1797212016 & -467071781328 \\
3 & -108 & 133488 & 368388 & -441631440 & -17699810580 & -467071781328 \\
4 & -10944 & -411264 & 75854232 & 4282520544 & 107873699400 & $*$ \\
5 & 61308 & -6824952 & -730782756 & -24887701224 & $*$ & $*$ \\
6 & 153828 & 74008728 & 4105498284 & $*$ & $*$ & $*$ \\
\hline
\end{tabular}

\begin{tabular}{|c|cccccc|}
\hline \multicolumn{7}{|c|}{$\mathrm{k}=3:$ Ooguri-Vafa Invariants $N_{k, m+n, k, n}$} \\
\hline$N_{3, m+n, 3, n}$ & $n=0$ & 1 & 2 & 3 & 4 & 5 \\
\hline$m=1$ & 0 & 0 & 0 & 0 & 0 & 0 \\
2 & 0 & 0 & 0 & 0 & 0 & 0 \\
3 & -8 & 0 & 0 & 192 & 0 & 0 \\
4 & 0 & 0 & 0 & 0 & 0 & $*$ \\
5 & 0 & 0 & 0 & 0 & $*$ & $*$ \\
6 & 4 & 0 & 0 & $*$ & $*$ & $*$ \\
\hline
\end{tabular}

Table 10. Ooguri-Vafa Invariants $N_{k, m+n, k, n}$ for $X_{9}^{(1,1,1,3,3)}$ with brane II at large volume. $k$ and $m$ label the class $t_{1}, t_{2}$ of $X_{9}$ and $n$ labels the brane winding. Blue entries agree with table 6 of [8] and entries $*$ exceed in this table the order of our calculation. 


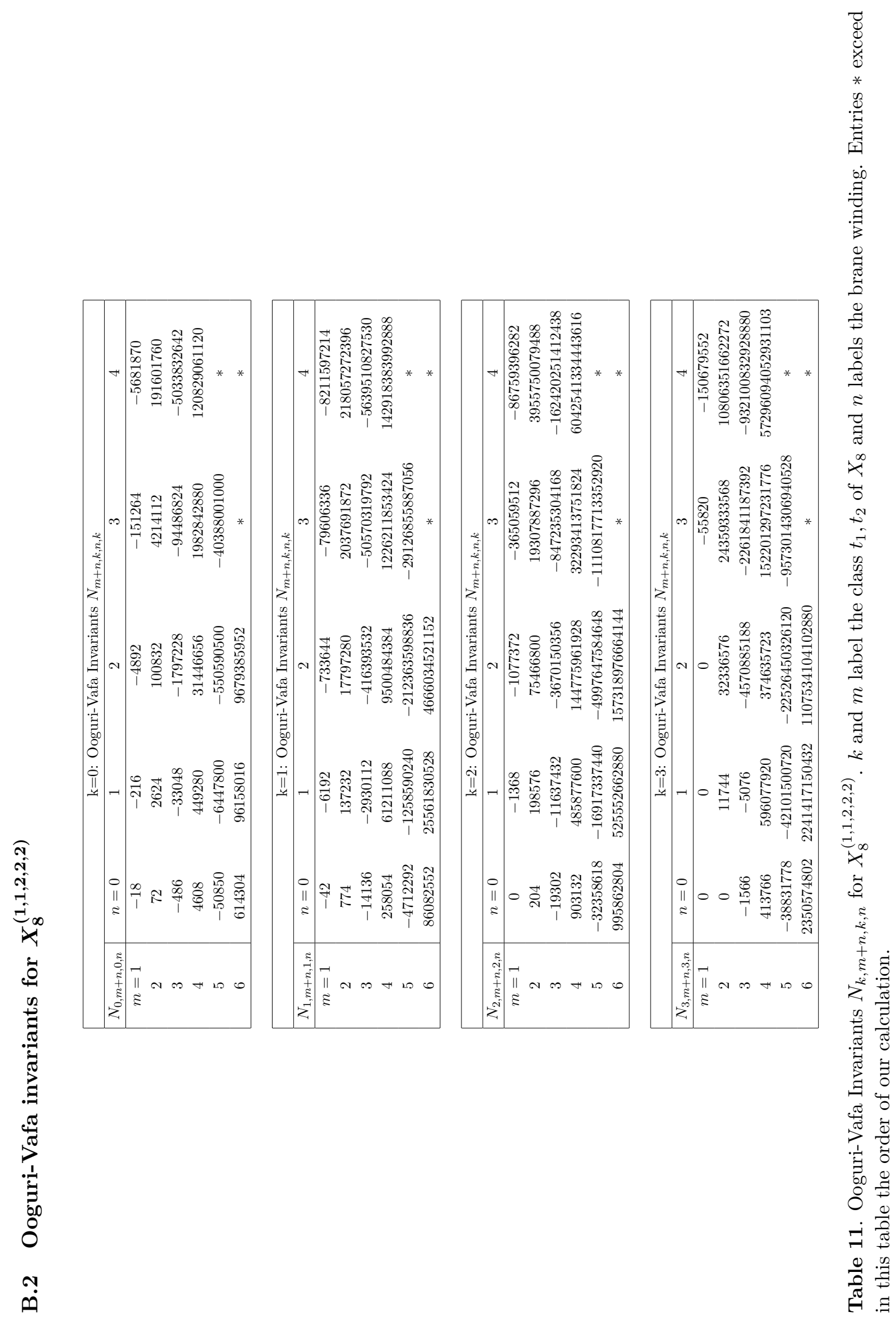




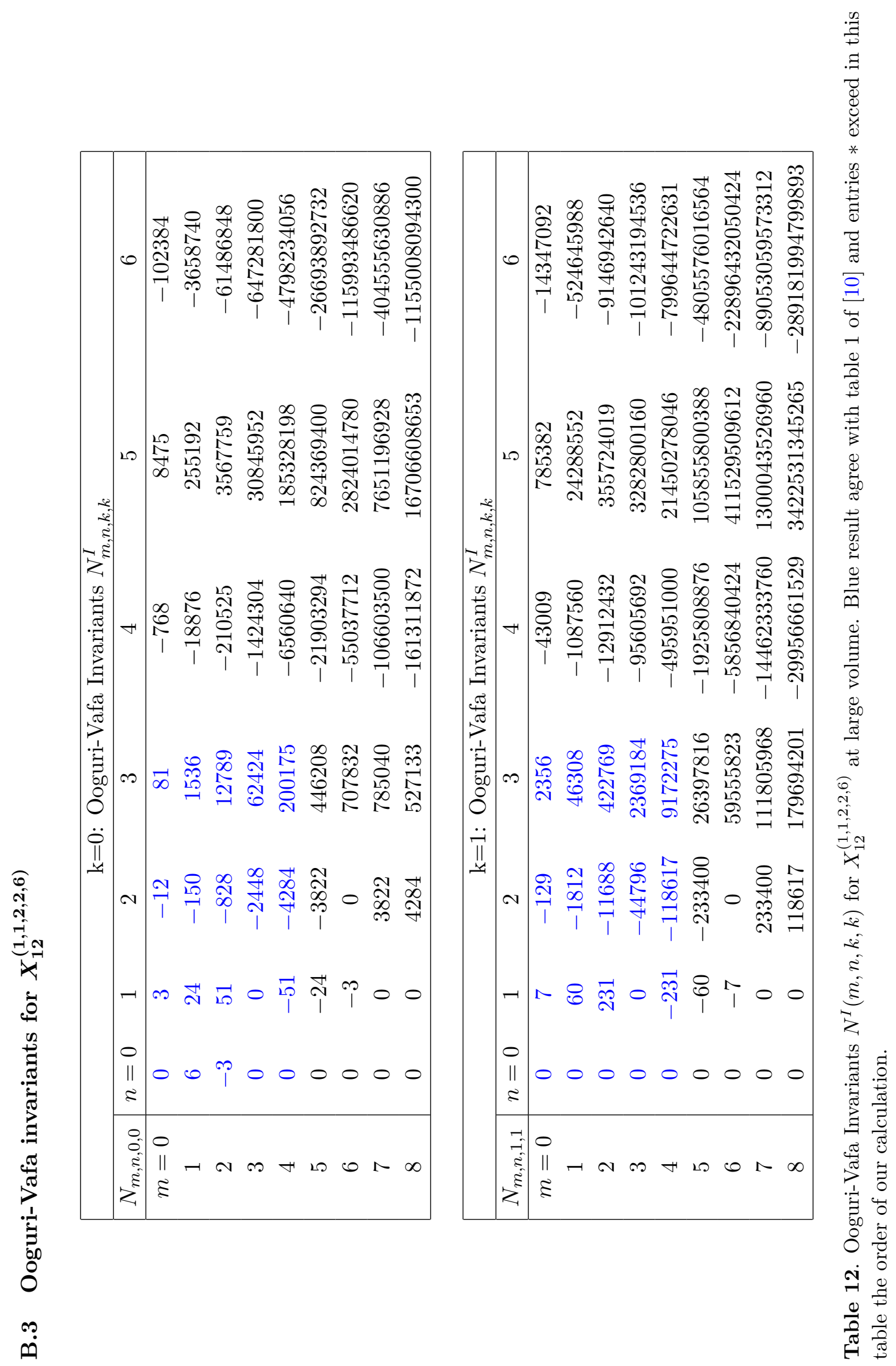



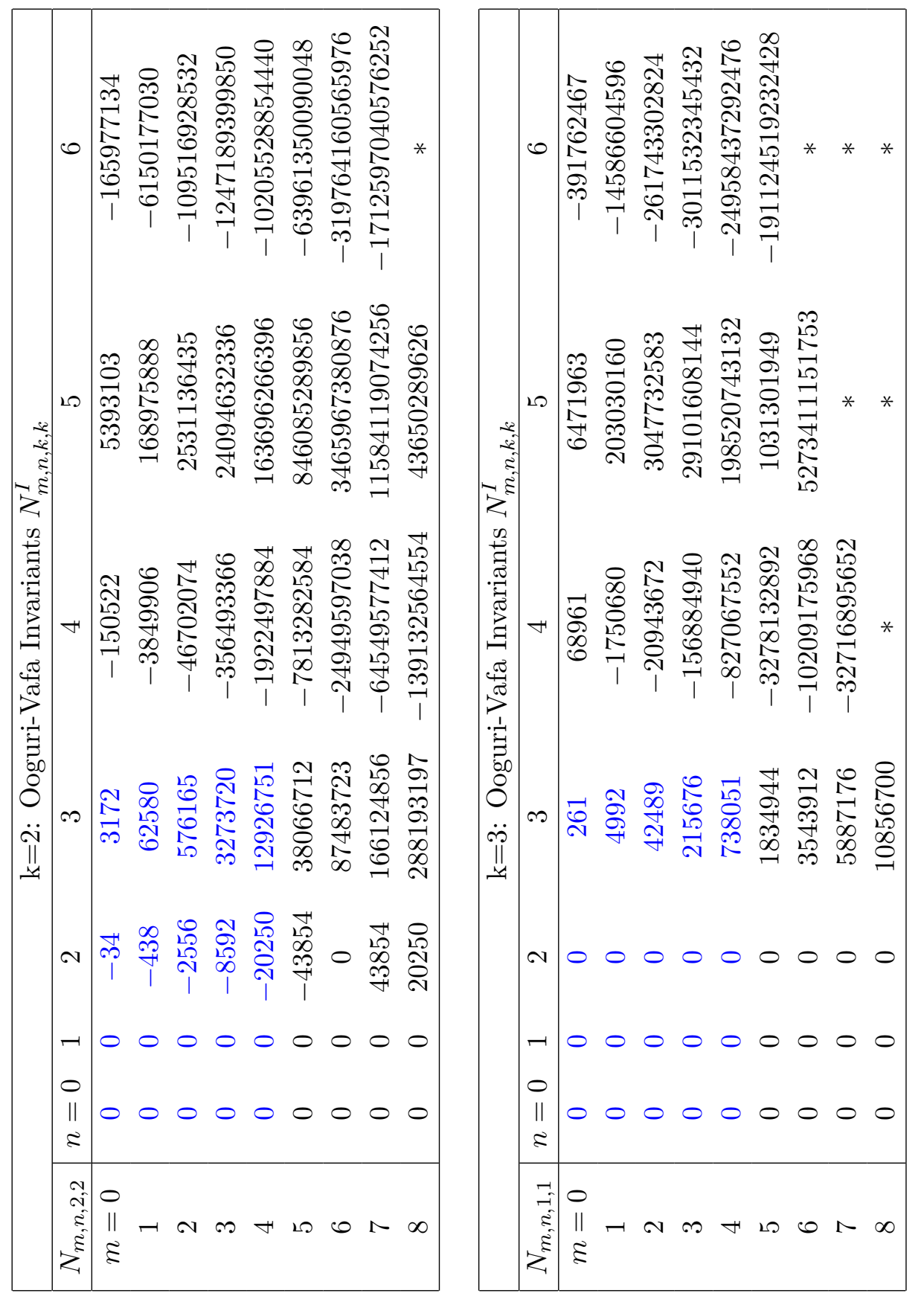

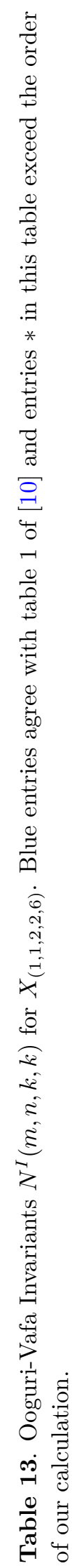



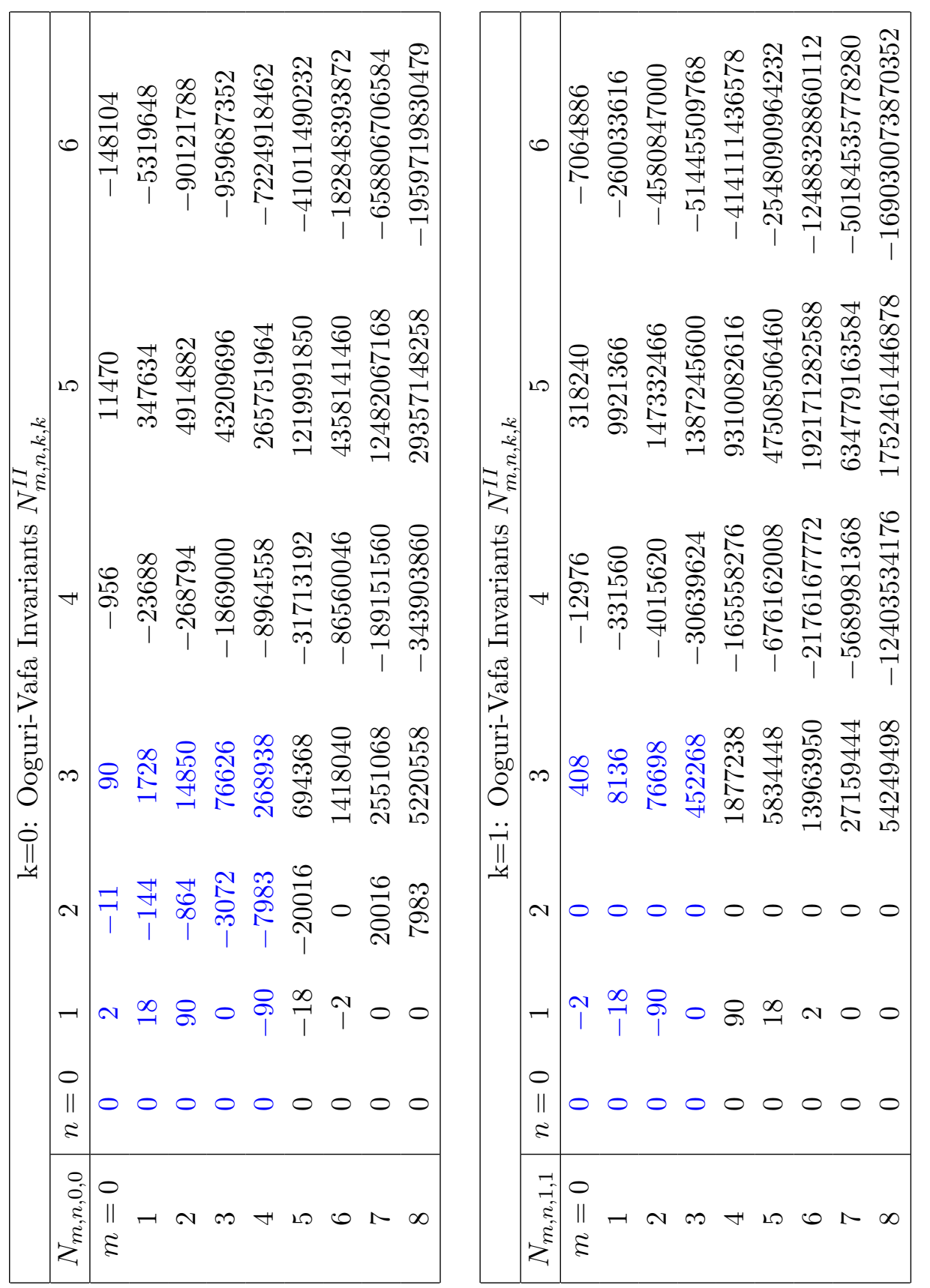

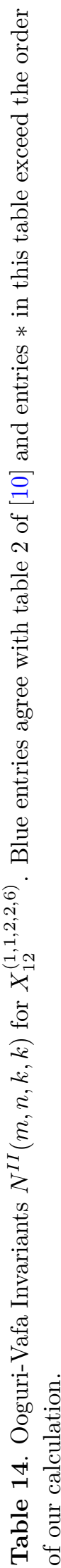




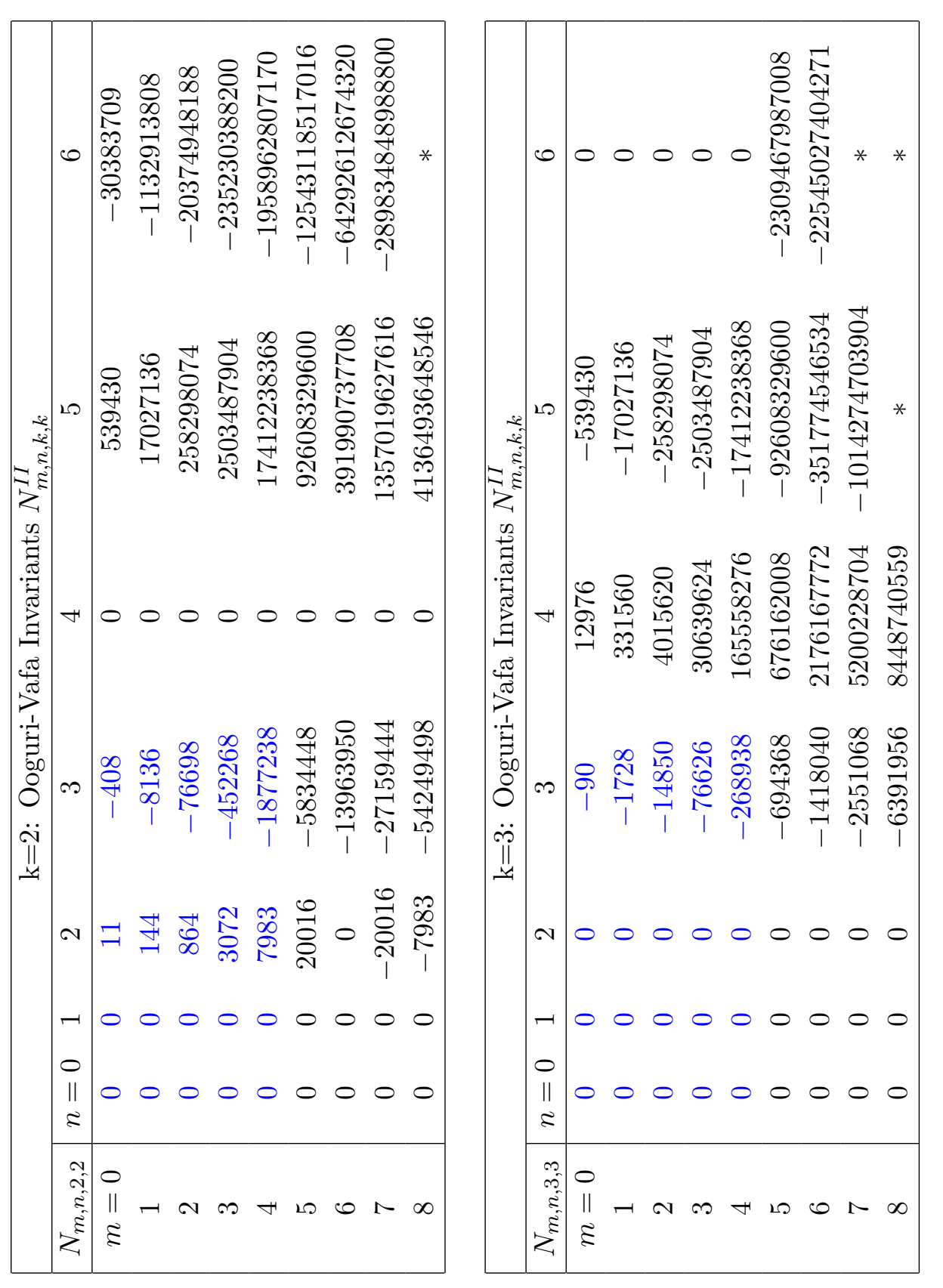

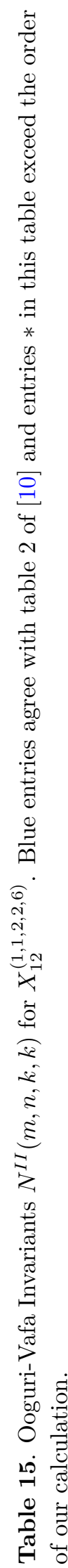




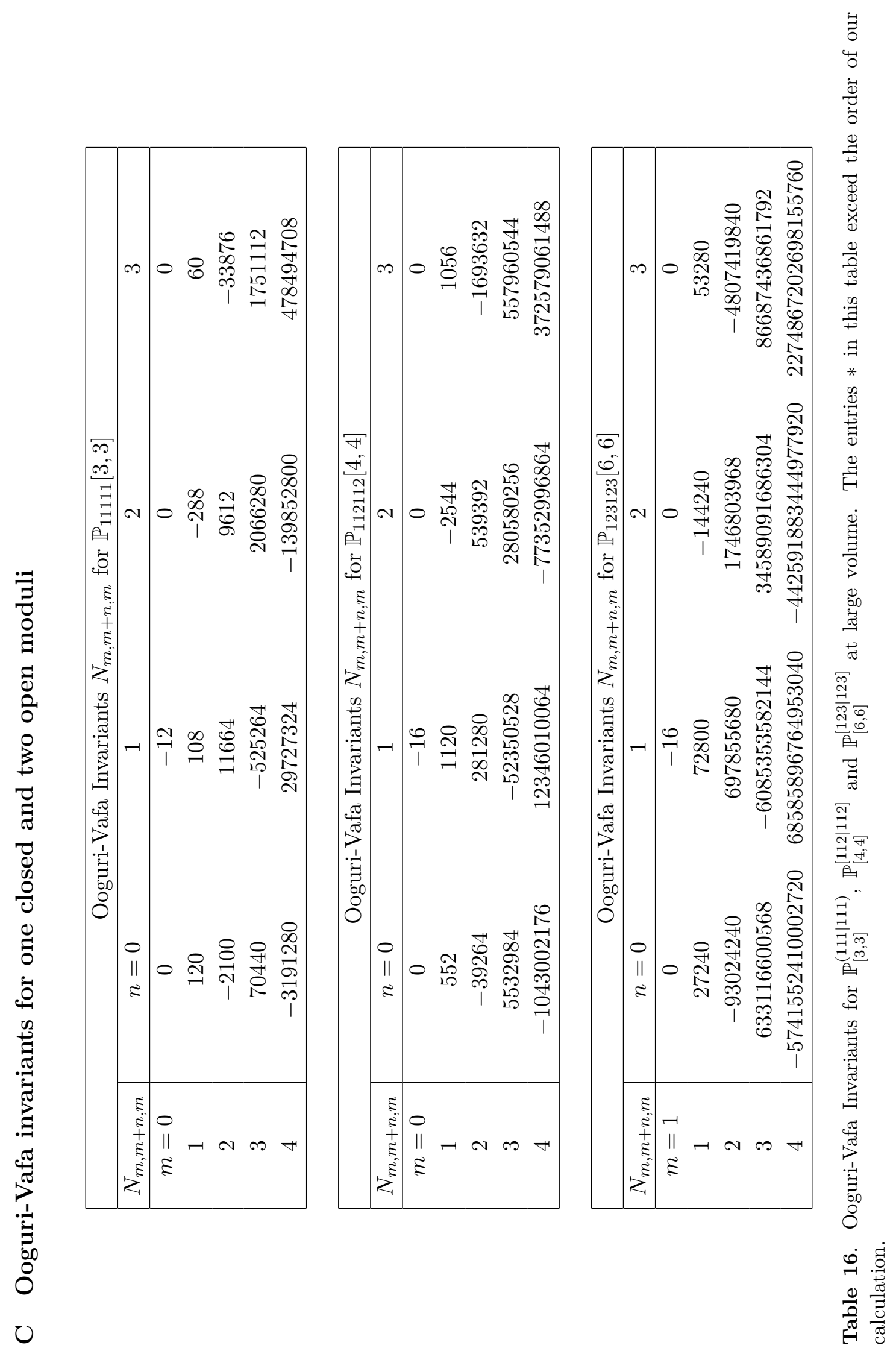




\section{Ooguri-Vafa invariants for one closed and three open moduli}

\begin{tabular}{|c|cccccc|}
\hline \multicolumn{7}{|c|}{$\mathrm{U}(1)$ Ooguri-Vafa Invariants $N_{m, m, m, n}$} \\
\hline$N_{m, m, m, n}$ & $n=0$ & 1 & 2 & 3 & 4 & 5 \\
\hline$m=0$ & 0 & 0 & 0 & 0 & 0 & 0 \\
1 & 0 & -4896 & 0 & 0 & 0 & 0 \\
2 & 0 & 0 & 1729728 & 0 & 0 & 0 \\
3 & 0 & 0 & 0 & -1530550656 & 0 & 0 \\
4 & 0 & 0 & 0 & 0 & 1882007669502 & 0 \\
\hline
\end{tabular}

\begin{tabular}{|c|cccccc|}
\hline \multicolumn{7}{|c|}{$\mathrm{U}(2)$ Ooguri-Vafa Invariants $N_{m, m, n}$} \\
\hline$N_{m, m, n}$ & $n=0$ & 1 & 2 & 3 & 4 & 5 \\
\hline$m=0$ & 0 & 0 & 0 & 0 & 0 & 0 \\
1 & 0 & 4832 & 0 & 0 & 0 & 0 \\
2 & 0 & 0 & 6266104 & 0 & 0 & 0 \\
3 & 0 & 0 & 0 & $\frac{55336185568}{3}$ & 0 & 0 \\
4 & 0 & 0 & 0 & 0 & 75779426010282 & -3443691801600 \\
\hline
\end{tabular}

Table 17. Ooguri-Vafa invariant of Sextic Hypersurface.

\begin{tabular}{|c|cccccc|}
\hline \multicolumn{7}{|c|}{ U(1) Ooguri-Vafa Invariants $N_{m, m, m, n}$} \\
\hline$N_{m, m, m, n}$ & $n=0$ & $n=1$ & $n=2$ & $n=3$ & $n=4$ & $n=5$ \\
\hline$m=0$ & 0 & 0 & 0 & 0 & 0 & 0 \\
1 & 0 & -3328 & 0 & 0 & 0 & 0 \\
2 & 0 & 0 & 2030336 & 0 & 0 & 0 \\
3 & 0 & 0 & 0 & -3115785728 & 0 & 0 \\
4 & 0 & 0 & 0 & 237081600 & $*$ & $*$ \\
\hline
\end{tabular}

\begin{tabular}{|c|cccccc|}
\hline \multicolumn{7}{|c|}{$\mathrm{U}(2)$ Ooguri-Vafa Invariants $N_{m, m, n}$} \\
\hline$N_{m, m, n}$ & $n=0$ & $n=1$ & $n=2$ & $n=3$ & $n=4$ & $n=5$ \\
\hline$m=0$ & 0 & 0 & 0 & 0 & 0 & 0 \\
1 & 0 & -5504 & 0 & 0 & 0 & 0 \\
2 & 0 & 0 & 9520016 & 0 & 0 & 0 \\
3 & 0 & 0 & 0 & $\frac{-430429613824}{15}$ & 0 & 0 \\
4 & 0 & 0 & 0 & 0 & $\frac{1000605023457608}{7}$ & -97447720529920 \\
\hline
\end{tabular}

Table 18. Ooguri-Vafa invariant of Octic Hypersurface. 
Open Access. This article is distributed under the terms of the Creative Commons Attribution License (CC-BY 4.0), which permits any use, distribution and reproduction in any medium, provided the original author(s) and source are credited.

\section{References}

[1] S.H. Katz and C.-C.M. Liu, Enumerative geometry of stable maps with Lagrangian boundary conditions and multiple covers of the disc, Adv. Theor. Math. Phys. 5 (2001) 1 [Geom. Topol. Monographs 8 (2006) 1] [math/0103074] [INSPIRE].

[2] J. Li and Y.S. Song, Open string instantons and relative stable morphisms, Adv. Theor. Math. Phys. 5 (2001) 67 [Geom. Topol. Monographs 8 (2006) 49] [hep-th/0103100] [InSPIRE].

[3] E. Witten, Chern-Simons gauge theory as a string theory, in Progress in Mathematics 133, Springer (1995), pp. 637-678 [hep-th/9207094] [INSPIRE].

[4] W. Lerche, P. Mayr and N. Warner, Holomorphic $N=1$ special geometry of open-closed type-II strings, hep-th/0207259 [INSPIRE].

[5] W. Lerche, P. Mayr and N. Warner, $N=1$ special geometry, mixed Hodge variations and toric geometry, hep-th/0208039 [INSPIRE].

[6] P. Mayr, $N=1$ mirror symmetry and open/closed string duality, Adv. Theor. Math. Phys. 5 (2002) 213 [hep-th/0108229] [INSPIRE].

[7] M. Aganagic and C. Vafa, Mirror symmetry, D-branes and counting holomorphic discs, hep-th/0012041 [INSPIRE].

[8] M. Alim, M. Hecht, P. Mayr and A. Mertens, Mirror Symmetry for Toric Branes on Compact Hypersurfaces, JHEP 09 (2009) 126 [arXiv:0901.2937] [INSPIRE].

[9] H. Jockers and M. Soroush, Effective superpotentials for compact D5-brane Calabi-Yau geometries, Commun. Math. Phys. 290 (2009) 249 [arXiv:0808.0761] [InSPIRE].

[10] H. Jockers and M. Soroush, Relative periods and open-string integer invariants for a compact Calabi-Yau hypersurface, Nucl. Phys. B 821 (2009) 535 [arXiv:0904.4674] [InSPIRE].

[11] T.W. Grimm, A. Klemm and D. Klevers, Five-Brane Superpotentials, Blow-Up Geometries and SU(3) Structure Manifolds, JHEP 05 (2011) 113 [arXiv:1011.6375] [INSPIRE].

[12] T.W. Grimm, T.-W. Ha, A. Klemm and D. Klevers, The D5-brane effective action and superpotential in $N=1$ compactifications, Nucl. Phys. B 816 (2009) 139 [arXiv:0811.2996] [INSPIRE].

[13] D. Cox, J. Little and H. Schenck, Toric Varieties, American Mathematical Society, Providence RI U.S.A. (2011).

[14] V.V. Batyrev, Dual polyhedra and mirror symmetry for Calabi-Yau hypersurfaces in toric varieties, J. Alg. Geom. 3 (1994) 493 [alg-geom/9310003] [INSPIRE].

[15] V.V. Batyrev and L.A. Borisov, On Calabi-Yau complete intersections in toric varieties, alg-geom/9412017 [INSPIRE].

[16] S. Hosono, B.H. Lian and S.-T. Yau, GKZ generalized hypergeometric systems in mirror symmetry of Calabi-Yau hypersurfaces, Commun. Math. Phys. 182 (1996) 535 [alg-geom/9511001] [INSPIRE]. 
[17] S. Hosono, A. Klemm, S. Theisen and S.-T. Yau, Mirror symmetry, mirror map and applications to Calabi-Yau hypersurfaces, Commun. Math. Phys. 167 (1995) 301 [hep-th/9308122] [INSPIRE].

[18] S. Hosono, A. Klemm, S. Theisen and S.-T. Yau, Mirror symmetry, mirror map and applications to complete intersection Calabi-Yau spaces, in AMS/IP Studies in Advanced Mathematics 1, American Mathematical Society, Providence RI U.S.A. (1996), pp. 545-606 [Nucl. Phys. B 433 (1995) 501] [hep-th/9406055] [INSPIRE].

[19] J. Stienstra, GKZ hypergeometric structures, math/0511351 [INSPIRE].

[20] D. Cox, Mirror symmetry and algebraic geometry, American Mathematical Society, Providence RI U.S.A. (1999).

[21] E. Witten, Phases of $N=2$ theories in two-dimensions, in AMS/IP Studies in Advanced Mathematics 1, American Mathematical Society, Providence RI U.S.A. (1996), pp. 143-211 [Nucl. Phys. B 403 (1993) 159] [hep-th/9301042] [INSPIRE].

[22] I.R. Shafarevich, Basic Algebraic Geometry 2, Springer (2016).

[23] A. Hatcher, Algebraic Topology, Cambridge University Press, Cambridge U.K. (2001).

[24] X.-T. Jiang and F.-Z. Yang, D-brane superpotentials, SU(2) Ooguri-Vafa invariants and TypeII/F -theory duality, arXiv:1710.06184 [INSPIRE].

[25] M. Artebani and I. Dolgachev, The hesse pencil of plane cubic curves, math/0611590. 